\title{
UNIFIED HYBRIDIZATION OF DISCONTINUOUS GALERKIN, MIXED AND CONTINUOUS GALERKIN METHODS FOR SECOND ORDER ELLIPTIC PROBLEMS
}

\author{
BERNARDO COCKBURN *, JAYADEEP GOPALAKRISHNAN ${ }^{\dagger}$, AND RAYTCHO LAZAROV $\ddagger$
}

\begin{abstract}
We introduce a unifying framework for hybridization of finite element methods for second order elliptic problems. The methods fitting in the framework are a general class of mixed-dual finite element methods including hybridized mixed, continuous Galerkin, non-conforming and a new, wide class of hybridizable discontinuous Galerkin methods. The distinctive feature of the methods in this framework is that the only globally coupled degrees of freedom are those of an approximation of the solution defined only on the boundaries of the elements. Since the associated matrix is sparse, symmetric and positive definite, these methods can be efficiently implemented. Moreover, the framework allows, in a single implementation, the use of different methods in different elements or subdomains of the computational domain which are then automatically coupled. Finally, the framework brings about a new point of view thanks to which it is possible to see how to devise novel methods displaying very localized and simple mortaring techniques, as well as methods permitting an even further reduction of the number of globally coupled degrees of freedom.
\end{abstract}

Key words. discontinuous Galerkin methods, mixed methods, continuous methods, hybrid methods, elliptic problems

AMS subject classifications. $65 \mathrm{~N} 30,65 \mathrm{M} 60$

Version of September 12, 2008

1. Introduction. We introduce a new unifying framework for hybridization of finite element methods for second order elliptic problems. This framework is unifying in the sense that it includes as particular cases hybridized versions of mixed methods [4, 11, 26], the continuous Galerkin (CG) method [31], and a new, wide class of hybridizable discontinuous Galerkin (DG) methods. The unifying framework allows to (i) significantly reduce the number of the globally coupled degrees of freedom of DG methods, (ii) use different methods in different parts of the computational domain and automatically couple them, and (iii) devise novel methods employing new mortaring techniques. We develop the unifying framework on the following model elliptic boundary value problem of second order written in mixed form,

$$
\begin{aligned}
\boldsymbol{q}+a \operatorname{grad} u & =0 & & \text { on } \Omega, \\
\operatorname{div} \boldsymbol{q}+d u & =f & & \text { on } \Omega, \\
u & =g & & \text { on } \partial \Omega .
\end{aligned}
$$

Here $\Omega \subset \mathbb{R}^{n}$ is a polyhedral domain $(n \geq 2), d(\boldsymbol{x})$ is a scalar nonnegative function, and $a(\boldsymbol{x})$ is a matrix valued function that is symmetric and uniformly positive definite on $\Omega$. In addition, we assume that the function $g$ is the restriction of a smooth scalar function on $\partial \Omega$, and that the functions $f, d$, and $a$ are smooth on $\bar{\Omega}$. These assumptions can be vastly generalized, but we take them for the sake of a transparent presentation of the design of our unifying framework.

1.1. The structure of the methods of the unifying framework. Let us begin the description of our results by arguing that what makes possible the construction of the unified framework is that all the numerical methods fitting in it are constructed by using a discrete version of a single property of the exact solution of the problem (1.1). This property is a characterization of the values of the exact solution $u$ on the interior boundaries of each of the elements $K$ of any triangulation of the domain $\Omega, \mathcal{T}_{h}$. Let us describe it.

If on the border of the element $K, \partial K$, we set $u=\lambda+g$, where

$$
\lambda=\left\{\begin{array}{ll}
u & \text { on } \partial K \backslash \partial \Omega, \\
0 & \text { on } \partial K \cap \partial \Omega,
\end{array} \text { and } g= \begin{cases}0 & \text { on } \partial K \backslash \partial \Omega, \\
g & \text { on } \partial K \cap \partial \Omega,\end{cases}\right.
$$

by the linearity of the problem, we have that

$$
(\boldsymbol{q}, u)=(\mathbf{Q} \lambda+\mathbf{Q} g+\mathbf{Q} f, \mathbf{U} \lambda+\mathrm{U} g+\mathrm{U} f) \quad \text { in } \Omega,
$$

${ }^{*}$ School of Mathematics, University of Minnesota, Vincent Hall, Minneapolis, MN 55455, USA, email: cockburn@math.umn.edu. Supported in part by the National Science Foundation (Grant DMS-0411254) and by the University of Minnesota Supercomputing Institute.

$\dagger$ Department of Mathematics, University of Florida, Gainesville, FL 32611-8105, USA, email: jayg@math.ufl.edu. Supported in part by the National Science Foundation (Grants DMS-0410030, DMS-0713833, and SCREMS-0619080).

${ }^{\ddagger}$ Department of Mathematics, Texas A\&M University, College Station, TX 77843-3368, U.S.A. email: lazarov@math.tamu.edu. Supported in part by the National Science Foundation (Grant NSF-DMS-0713829 and NSF-CNS-ITR-0540136) 
where the so-called local solvers $(\mathbf{Q}(\cdot), \mathrm{U}(\cdot))$ are defined on the element $K \in \mathcal{T}_{h}$ as follows. For any single-valued functions $\mathrm{m}$ on $L^{2}(\partial K)$ and $f$ on $L^{2}(K)$, the functions $(\mathbf{Q m}, \mathrm{Um})$ and $(\mathbf{Q} f, \mathrm{U} f)$ are the solutions of

$$
\begin{aligned}
& c \mathbf{Q m}+\operatorname{grad} U \mathrm{~m}=0, \quad \operatorname{div} \mathbf{Q m}+d \mathrm{Um}=0, \quad \text { on } K, \quad \mathbf{U m}=\mathbf{m} \quad \text { on } \partial K, \\
& c \mathbf{Q} f+\operatorname{grad} \cup f=0, \quad \operatorname{div} \mathbf{Q} f+d \mathbf{U} f=f, \quad \text { on } K, \quad \mathbf{U} f=0 \quad \text { on } \partial K,
\end{aligned}
$$

where $c=a^{-1}$, for each element $K \in \mathcal{T}_{h}$.

Conversely, the above property holds if and only if, see, for example, [46], the normal component of $\mathbf{Q} \lambda+$ $\mathbf{Q} g+\mathbf{Q} f$ across interelement boundaries is continuous. We thus see that this transmission condition, which we formally express as

$$
\llbracket \mathbf{Q} \lambda+\mathbf{Q} g+\mathbf{Q} f \rrbracket=0,
$$

completely characterizes the function $\lambda$. Here $\llbracket \rrbracket$ denotes the jump of the normal component of the a vector accross $\partial K$.

The finite element methods of the unified framework are those that can be expressed as a discrete version of the above property. In this way, the only globally coupled degrees of freedom are bound to be those describing the approximation to $\lambda$. Thus, each of those method provides an approximate solution of the form

$$
\left(\boldsymbol{q}_{h}, u_{h}\right)=\left(\mathbf{Q} \lambda_{h}+\mathbf{Q} g_{h}+\mathbf{Q} f, \mathfrak{U} \lambda_{h}+\mathcal{U} g_{h}+\mathcal{U} f\right)
$$

where $\lambda_{h}$, resp., $g_{h}$, is an approximation in some finite dimensional space $M_{h}$, resp., $\mathbf{M}_{h}$, of the values of $u$ on the faces of the elements lying in the interior, resp., in the border, of $\Omega$; and $(\mathbf{Q m}, \mathcal{U} \mathrm{m})$ and $(\mathbf{Q} f, \mathcal{U} f)$ are discrete versions of the exact local solvers (1.4) - we keep the same notation for the sake of simplicity. Moreover, the methods are such that $\lambda_{h}$ can be determined by a discrete version of the transmission condition (1.5) which we write as follows:

$$
a_{h}\left(\lambda_{h}, \mu\right)=b_{h}(\mu) \quad \text { for all } \mu \in M_{h} .
$$

In [26], where the hybridization of mixed methods was considered, the equation determining $\lambda_{h}$ was called the jump condition. In our setting, it is called the conservativity condition to reflect the incorporation into the framework of DG and CG methods.

Note that all the methods in the unified framework provide approximations for $(\boldsymbol{q}, u)$ in the interior of the elements $K \in \mathcal{T}_{h},\left(\boldsymbol{q}_{h}, u_{h}\right)$, as well as an approximation of $u$ on the interior border of the elements, $\lambda_{h}$; this is why they are called hybrid. This is in agreement with the definition of hybrid methods proposed in [22, p. 421]: "we may define more generally as a hybrid method any finite element method based on a formulation where one unknown is a function, or some of its derivatives, on the set $\Omega$, and the other unknown is the trace of some of its derivatives of the same function, or the trace of the function itself, along the boundaries of the set $K$." Here $K$ denotes a typical element of the triangulation. A long list of hybrid methods can be found in [22, 12, 51].

Of course, not every finite element method displays the above roughly described structure; in particular, it might not even be a hybrid method. However, many such methods can be rewritten as hybrid methods; this process is what can be called the hybridization of a finite element method. We say that we can hybridize a given finite element method if we can find a hybrid method (part) of whose solution coincides with the solution of the given method. The original finite element method is called hybridizable, and the hybrid method is then said to be a hybridization of the original method; for short, we call it a hybridized method. Next, we give a brief overview of the hybridization techniques of relevance for our purposes.

1.2. Hybridization of finite element methods. The first hybridization of a finite element method was proposed in 1965 [39] for a numerical method for solving the equations of linear elasticity. Perhaps because it was then intended as an implementation technique, the distinction between hybridization and static condensation, a widely known algebraic manipulation for size reduction of already assembled matrices, is seldom made in the engineering literature. However, in 1985 [4], hybridization was shown to be more than an implementation trick as it was proven that the new unknown $\lambda_{h}$, also interpreted to be the Lagrange multiplier associated with a continuity condition on the approximate flux, contains extra information about the exact solution. This was used to enhance the accuracy of the approximation by means of a local postprocessing [4, 11, 35]; see also [10].

After yet another two decades, a new perspective on hybridization emerged [26] and the characterization of the approximate trace $\lambda_{h}$ as the solution of the weak formulation (1.7) was introduced; this was done in 
the setting of the hybridization of the RT and BDM mixed methods of arbitrary degree. The special case of the lowest order RT method had been previously considered in [21] within the framework of a study of the equivalence of mixed and nonconforming methods. In [26], it was shown that the formulation (1.7) not only simplifies the task of assembling the stiffness matrix for the multiplier but can be used to establish unsuspected links between apparently unrelated mixed methods. It was also shown that it allows the devising and analysis of new, variable degree versions of those methods [27].

This new hybridization approach was later extended to finite element methods for the stationary Stokes equations using spaces of exactly divergence-free velocities; it was intended as an effective technique to bypass the extremely difficult construction of such spaces. It was successfully applied to a discontinuous Galerkin method [15] and to a mixed method for Stokes flow [28, 29]. For a review of these results, see [30]. Recently [31], this hybridization approach was applied to the CG method to pave the way for the computation of an $H$ (div)conforming approximation of the flux from the CG solution.

1.3. Hybridization of DG methods. In this paper, we continue this effort and show how to hybridize a large class of DG methods. Thus, we show that their approximate solution $\left(\boldsymbol{q}_{h}, u_{h}\right)$ can be expressed as in (1.6) and that the approximate trace $\lambda_{h}$, which is nothing but the so-called numerical trace $\widehat{u}_{h}$ on the inter-element boundaries, see [5], satisfies the weak formulation (1.7). In other words, we identify a class of DG methods whose globally coupled degrees of freedom are those of the numerical trace $\widehat{u}_{h}$ only; this results in an efficient implementation of these methods, as we argue below. In this way, the main disadvantages of DG methods for elliptic problems compared to other methods, namely, a higher number of globally coupled degrees of freedom for the same mesh and a lower sparsity of the corresponding stiffness matrices, are eliminated to a significant extent.

The simplest examples of such methods are obtained by using a DG method to define the local solvers and by taking what could be called the corresponding natural choice for the space $M_{h}$ for the approximate trace $\lambda_{h}$. For example, we can use the local discontinuous Galerkin (LDG) method to define the local solvers and construct a hybridizable DG method. Surprisingly, it turns out that the resulting DG method is not an LDG method but one of the DG methods considered in [17]; see Corollary 3.2. A similar result holds for the hybridizable DG methods whose local solvers are the interior penalty (IP) method, that is, the resulting method is not the original IP method but the IP-like method considered in [38]; see Corollary 3.4. This is in sharp contrast with the RT, BDM and CG methods each of which can be hybridized by using as local solvers the RT, $\mathrm{BDM}$ and CG methods, respectively.

It is interesting to note that the only known DG methods that turn out to be hybridizable by our technique are the following: a subset of the methods considered in [17], the minimal dissipation DG methods considered in [20], the minimal dissipation LDG method analyzed in [24], and the DG method considered in [38] and then rewritten as an IP method in [37]. With the exception of some LDG methods, none of the DG methods considered in the unified analysis of DG methods carried out in [5] is a hybridizable DG method. The reason is, roughly speaking, as follows. For all methods considered in [5], the variable $\boldsymbol{q}_{h}$ is easily eliminated from the equations due to the fact that the numerical trace $\widehat{u}_{h}$ is independent of $\boldsymbol{q}_{h}$ or $\operatorname{grad} u_{h}$; a primal formulation can then be found solely in terms of $u_{h}$. In contrast, in our approach, we eliminate both $\boldsymbol{q}_{h}$ and $u_{h}$ from the equations and obtain a formulation in terms of $\widehat{u}_{h}$ only, namely, equation (1.7). For this, it turns out that we need $\widehat{u}_{h}$ to be dependent on $\boldsymbol{q}_{h}$ or grad $u_{h}$, except for a few special LDG methods.

1.4. Properties of the algebraic system of hybridizable DG methods. As pointed out above, since the degrees of freedom of the functions $\mu$ in the finite element space $M_{h}$ are associated with the borders of the elements only, the stiffness matrix associated with the weak formulation (1.7) of the numerical trace $\widehat{u}_{h}=\lambda_{h}$ is significantly smaller than the one associated to the original variables $\left(\boldsymbol{q}_{h}, u_{h}\right)$. Moreover, the actual computation of the approximate solution of DG methods becomes competitive with that of hybridized mixed methods. For example, as we show below, on triangulations made of simplexes the stiffness matrix associated with the weak formulation (1.7) of any hybridizable DG method has the same size, block-structure, and sparsity as the corresponding hybridized BDM [11] and RT [49] mixed methods; see [26] for details. Even more, it was recently proved, see [25, Property (iii) of Theorem 2.4], that the stiffness matrices of the hybridized BDM, RT methods and the so-called single face hybridizable DG method are in fact identical provided $d=0$.

1.5. New automatic coupling of different methods and mortaring techniques. One of the main features of the unified framework is that it allows for a single implementation of a vast class of finite element methods including DG, mixed, nonconforming, and CG methods, and for their automatic coupling. Since it 
can be done even in the presence of non-matching meshes, the unified framework provides a novel coupling and mortaring technique. This induces a paradigm shift in the way we view different finite element methods fitting in the framework, especially when considering adaptive algorithms. Indeed, since all these methods can be implemented within a single framework, the issue is now to investigate which method to use in what part of the domain in order to fully exploit its individual advantages.

Let us briefly compare our new mortaring technique with the already established ones. Mortaring techniques (see the pioneering work [9]) were introduced to accommodate methods that can be defined in separate subdomains that could have been independently meshed. This technique introduces an auxiliary space for a Lagrange multiplier associated with a continuity constraint on the approximate solution. The resulting system could be written either as a saddle point problem, symmetric but indefinite [8], or as a non-conforming finite element approximation, which leads to a symmetric positive definite system; see, for example, [9, 41]. This classical mortaring is a powerful technique to achieve flexibility in the meshing and the choice of the finite element approximation. The work in this direction includes also coupling of mixed and CG [53], mixed and mixed FEM [2, 45], and DG and mixed methods [40].

However, this mortaring approach is very different from ours since, instead of enforcing the continuity of the approximation to $u$, we enforce a continuity condition on the approximation to the flux $\boldsymbol{q}$. The way of coupling and mortaring provided by the unified framework represents a simpler alternative to the above mentioned mortaring techniques as well as to earlier works on the coupling of CG and DG methods implicitly contained in [5] and explicitly emphasized in [48], as well as to the coupling of DG and mixed methods introduced in [23] and in [50].

1.6. Devising new methods. The unified framework provides a new point of view for constructing new methods. We provide three main examples of such methods. The first one is a family of methods well suited for $h p$-adaptivity and for dealing with non-matching meshes. On each element $K \in \mathcal{T}_{h}$, it uses local solvers obtained from the RT, BDM, LDG or CG methods by means of a suitable modification of the definition of the numerical trace of the flux of some faces of $K$ only. For example, by modifying the numerical trace of the CG- $\mathrm{H}$ method on the element faces lying on the non-matching interface, we allow the method to handle non-matching grids. This method represents an alternative to the coupling of DG and CG methods proposed in [48].

The second example is a variable-degree RT method that can be used on some classes of nonconforming meshes. The third example is called the embedded DG (EDG) method; it was introduced in the setting of shell problems in [43]. An EDG method is obtained from an already existing hybridizable method by simply modifying the space $M_{h}$. This capability can be used as a new mortaring technique for dealing with nonmatching meshes, as we are going to see. Moreover, some EDG methods give rise to a stiffness matrix whose size and sparsity is exactly equal to that of the statically condensed stiffness matrix of the CG method while retaining the stabilization mechanisms typical of DG methods; see [43]. As a consequence, the EDG methods can immediately be incorporated into existing commercial codes. Related to the EDG methods are the so-called multiscale DG methods $[44,14]$ which were introduced with a similar intention but a different approach.

1.7. Possibilities and recent developments. The unified framework could be used to establish a single $a$ priori and a single a posteriori error analysis of all the methods fitting in it. It could be used to compare different methods or to establish new relations between them just as the unsuspected relation between the RaviartThomas (RT) and the Brezzi-Douglas-Marini (BDM) methods in [26] was recently uncovered by comparing their hybridized versions. The framework could also be used to further explore the relation between mixed and nonconforming methods like the relation between the RT method of lowest order and a nonconforming method established in [4] and exploited in [47]. This work was later generalized in [1] where links between a variety of mixed and nonconforming methods were established; see also the references therein. Finally, the unifying framework can be used to devise new preconditioners based on, for example, substructuring techniques. However, in this paper, none of the above-mentioned issues will be investigated.

On the other hand, several discoveries induced by the unifying framework have already taken place. In particular, new DG methods which are more accurate and efficient than any other known DG method have been uncovered. Indeed, by exploiting the structure of the unified framework, a new DG method, called the single face, hybridizable (SFH) DG method, was constructed which lies in between the RT and BDM methods; see [25]. It is the first known DG method, using polynomials of degree $k$ for both $\boldsymbol{q}_{h}$ and $u_{h}$, proven to converge with order $k+1$ in both variables; all other DG methods converge with order $k$ in the flux only. Moreover, the SFH method shares with the RT and BDM methods their remarkable superconvergence properties; this allow 
for the element-by-element computation of a new approximation $u_{h}^{\star}$ converging with order $k+2$. These results were then extended to other hybridizable DG methods in [33]. Therein, it was shown that in order to achieve the above-mentioned convergence properties, the inter-element jumps of both unknowns have to be penalized essentially in the same way. This goes against the established belief that the inter-element jumps of $u_{h}$ need to be strongly penalized while the inter-element jumps of $\boldsymbol{q}_{h}$ need not be.

Also recently, a study of the EDG methods obtained from hybridizable DG methods by forcing the numerical trace to be continuous has been carried out in [32]. It was proven that these EDG methods lose the abovementioned convergence properties because the numerical trace $\widehat{\boldsymbol{q}}_{h}$ is not single valued. Moreover, numerical evidence was provided indicating that this loss of accuracy of the EDG method is not compensated by the computational advantage of having a reduced amount of globally coupled degrees of freedom. The hybridizable DG methods, with properly chosen penalization parameters, are thus more efficient than their EDG counterparts.

1.8. Organization of the paper. The paper is organized as follows. In Section 2 , we describe the general structure of the hybridized finite element methods and prove that the approximate trace $\lambda_{h}$ is characterized as the solution of a weak formulation of the form (1.7); see Theorem 2.1. We then provide sufficient conditions for the existence and uniqueness of the solution $\lambda_{h}$; see Theorem 2.4. Further in this section we give some implementation details and compare the memory requirements of the hybridizable methods with those of some classical DG methods. In Section 3, we give several examples of hybridizable finite element methods. These include mixed methods using RT and BDM finite element spaces, a large variety of DG, the CG and some nonconforming finite element methods. In Section 4, we build on the results of the previous section and construct the above mentioned novel hybridizable methods. Finally, in Section 5, we conclude the paper with a few extensions and some final remarks.

2. The general framework of hybridization. In this section, we display the structure of the hybridized finite element methods for the second-order elliptic problem (1.1). We begin by presenting the exact definition of the linear forms appearing in the weak formulation of the form (1.7) determining the approximate trace $\lambda_{h}$. We then provide sufficient conditions for the existence and uniqueness of $\lambda_{h}$, and show that the assembly of the corresponding matrix equation can be done in a typical finite element fashion. We end by describing the sparsity structure of the stiffness matrix and comparing it with that of the stiffness matrices of the hybridized RT, the IP and the LDG methods.

2.1. Notation. We use the notation used in [5]; let us recall it. Let $\mathcal{T}_{h}$ be a collection of disjoint elements that partition $\Omega$. The shape of the elements is not important in this general framework. Moreover, the triangulation $\mathcal{T}_{h}$ need not be conforming (we say that a triangulation $\mathcal{T}_{h}$ is conforming if whenever the intersection of the boundaries of any two elements has non-zero $(n-1)$-Lebesgue measure, the intersection is a face of each of the elements). So, $\mathcal{T}_{h}$ can be a collection of simplices, quadrilaterals, cubes, or a mixture of them which are not required to align across element interfaces. An interior "face" of $\mathcal{T}_{h}$ is any planar set $e$ of positive $(n-1)$-dimensional measure of the form $e=\partial K^{+} \cap \partial K^{-}$for some two elements $K^{+}$and $K^{-}$of the collection $\mathcal{T}_{h}$. (We use the word "face" even when $n=2$.) We say that $e$ is a boundary face if there is an element $K$ of $\mathcal{T}_{h}$ such that $e=\partial K \cap \partial \Omega$ and the $(n-1)$-Lebesgue measure of $e$ is not zero. Let $\mathcal{E}_{h}^{\circ}$ and $\mathcal{E}_{h}^{\partial}$ denote the set of interior and boundary faces of $\mathcal{T}_{h}$, respectively. We denote by $\mathcal{E}_{h}$ the union of all the faces in $\mathcal{E}_{h}^{\circ}$ and $\mathcal{E}_{h}^{\partial}$. In all our examples, elements of $\mathcal{E}_{h}^{\circ}$ and $\mathcal{E}_{h}^{\partial}$ are affine sets, although that is not required for the considerations in this section.

Finite element methods based on the mesh $\mathcal{T}_{h}$ typically use some finite dimensional polynomial approximation spaces on each element of $\mathcal{T}_{h}$. On an element $K$, we denote by $\boldsymbol{V}(K)$ the polynomial space in which the flux $\boldsymbol{q}$ is approximated and by $W(K)$ the space in which the scalar solution $u$ is approximated. The corresponding global finite element spaces are defined by

$$
\boldsymbol{V}_{h}=\left\{\boldsymbol{v}:\left.\boldsymbol{v}\right|_{K} \in \boldsymbol{V}(K)\right\} \quad \text { and } \quad W_{h}=\left\{w:\left.w\right|_{K} \in W(K)\right\} .
$$

On an interior face $e=\partial K^{+} \cap \partial K^{-}$, we consider scalar and vector functions that are in general double valued. For any discontinuous (scalar or vector) function $q$ in $W_{h}$ or $\boldsymbol{V}_{h}$, the trace $\left.q\right|_{e}$ is a double-valued function, whose two branches are denoted by $\left(\left.q\right|_{e}\right)_{K^{+}}$and $\left(\left.q\right|_{e}\right)_{K^{-}}$. To simplify the notation, we often shorten these to $q_{K^{+}}$and $q_{K^{-}}$, respectively. These branches are defined by $q_{K^{ \pm}}(\boldsymbol{x})=\lim _{\epsilon \downarrow} q\left(\boldsymbol{x}-\epsilon \boldsymbol{n}_{K^{ \pm}}\right)$for all $\boldsymbol{x}$ in $e$. Here and elsewhere, $\boldsymbol{n}$ denotes the double-valued function of unit normals on $\mathcal{E}_{h}$, so on any face $e \subseteq \partial K, \boldsymbol{n}_{K}$ denotes the unit outward normal of $K$. Same notations are used for vector functions. For any double-valued vector 
function $\boldsymbol{r}$ on an interior face $e$, we define the jump of its normal component across the face $e$ by

$$
\llbracket \boldsymbol{r} \rrbracket_{e}:=\boldsymbol{r}_{K^{+}} \cdot \boldsymbol{n}_{K^{+}}+\boldsymbol{r}_{K^{-}} \cdot \boldsymbol{n}_{K^{-}}
$$

On any face $e$ of $K$ lying on the boundary, we set

$$
\llbracket \boldsymbol{r} \rrbracket_{e}:=\boldsymbol{r}_{K} \cdot \boldsymbol{n}_{K}
$$

To simplify the exposition, we use $\llbracket \boldsymbol{r} \rrbracket$ to denote the single valued function on the entire set $\mathcal{E}_{h}$ which is equal to $\llbracket \boldsymbol{r} \rrbracket_{e}$ on every face $e \in \mathcal{E}_{h}$. Similarly, for any $e \in \mathcal{E}_{h}^{\circ}$ we define

$$
\left\{\{\xi\}_{e}=\frac{1}{2}\left(\xi_{K^{+}}+\xi_{K^{-}}\right), \quad\left\{\{\boldsymbol{q}\}_{e}=\frac{1}{2}\left(\boldsymbol{q}_{K^{+}}+\boldsymbol{q}_{K^{-}}\right), \quad \llbracket \xi \rrbracket_{e}=\xi_{K^{+}} \boldsymbol{n}_{K^{+}}+\xi_{K^{-}} \boldsymbol{n}_{K^{-}} .\right.\right.
$$

For a boundary face $e$ in $\mathcal{E}_{h}^{\partial}$, the operator $\left\{\{\cdot\}_{e}\right.$ is also considered to be the identity, so that we can put together the local operators $\left\{\{\cdot\}_{e}\right.$ to form a global operator $\left\{\{\cdot\}\right.$ on $\mathcal{E}_{h}$ just as we did for $\llbracket \cdot \rrbracket$.

Our notation for inner products is standard: For functions $u$ and $v$ in $L^{2}(D)$, we write $(u, v)_{D}=\int_{D} u v d x$, if $D$ is a domain of $\mathbb{R}^{n}$ and $\langle u, v\rangle_{D}=\int_{D} u v d x$, if $D$ is a domain of $\mathbb{R}^{n-1}$. To emphasize the mesh dependent nature of certain integrals, we introduce the notation

$$
(v, w)_{\mathcal{T}_{h}}=\sum_{K \in \mathcal{T}_{h}}(v, w)_{K} \quad \text { and } \quad\langle\mu, \lambda\rangle_{\mathcal{E}}=\sum_{e \in \mathcal{E}}\langle\mu, \lambda\rangle_{e},
$$

for functions $v, w$ and $\mu, \lambda$ defined on $\Omega$ and $\mathcal{E}_{h}$, respectively. Here $\mathcal{E}$ is any subset of $\mathcal{E}_{h}$.

2.2. The general structure of the methods. To describe the structure of the methods fitting in the unified framework, we mimic the characterization of the exact solution given in the Introduction.

Thus, we begin by choosing the space $\mathrm{M}_{h}$ of approximate traces, by taking the approximation to $\lambda, \lambda_{h}$, in

$$
M_{h}:=\left\{\mu \in \mathrm{M}_{h}: \mu=0 \text { on } \partial \Omega\right\}
$$

and by setting $g_{h}=I_{h} g$, where $I_{h}$ is a suitably defined interpolation operator with image in M $\mathrm{M}_{h}$ Recall that $g$ is the extension by zero of the Dirichlet data on $\partial \Omega$ to $\mathcal{E}_{h}^{\circ}$; see (1.2).

Next, we introduce a discrete version of the local solvers (1.4a) and (1.4b). The first local solver maps each function $\mathrm{m}$ in $\mathrm{M}_{h}$ to the function $(\mathbf{Q m}, \mathcal{U} \mathrm{m})$ on $\Omega$ whose restriction to any mesh element $K$ is in $\boldsymbol{V}(K) \times W(K)$ and satisfies the following discretization of $(1.4 \mathrm{a})$ :

$$
\begin{aligned}
& (c \boldsymbol{Q m}, \boldsymbol{v})_{K}-(\mathcal{U}, \operatorname{div} \boldsymbol{v})_{K}=-\langle\mathrm{m}, \boldsymbol{v} \cdot \boldsymbol{n}\rangle_{\partial K}, \quad \text { for all } \boldsymbol{v} \in \boldsymbol{V}(K), \\
& -(\operatorname{grad} w, \mathbf{Q} \mathrm{m})_{K}+\langle w, \widehat{\mathbf{Q}} \mathrm{m} \cdot \boldsymbol{n}\rangle_{\partial K}+\left(d \mathcal{U}_{\mathrm{m}}, w\right)_{K}=0 \text {, for all } w \in W(K) \text {. }
\end{aligned}
$$

Here $\widehat{\mathbf{Q}} \mathrm{m}$ represents the numerical trace of the flux, which is in general a double-valued function on $\mathcal{E}_{h}^{\circ}$. In inner products involving $\widehat{\mathbf{Q}} \mathrm{m}$ over a single simplex boundary $\partial K$, the integrand is assumed to be the branch $(\widehat{\mathbf{Q}} \mathrm{m})_{K}$ from that simplex. In all examples we consider in this paper, the numerical flux $\widehat{\mathbf{Q}} \mathrm{m}$ is either expressed explicitly in terms of $(\mathbf{Q m}, \mathcal{U} \mathrm{m})$, or is a unknown function. In the examples where the latter case arises, we introduce the space in which the unknown $\widehat{\underline{Q}} \mathrm{~m}$ lies, and add new equations to render the resulting formulation uniquely solvable. At this point, however, the precise definition of $\widehat{\mathbf{Q}} \mathrm{m}$ is not essential as we are solely interested in displaying the structure of the method for any $\widehat{\mathbf{Q}} \mathrm{m}$. Below we formally require $\mathrm{m} \mapsto(\mathbf{Q} \mathrm{m}, \widehat{\mathbf{Q}} \mathrm{m}, \mathcal{U} \mathrm{m})$ to be a well defined linear map; see Assumption 2.1.

The second local solver is a discretization of the second boundary value problem in (1.4b). It associates to any $f \in L^{2}(\Omega)$ the pair $(\mathbf{Q} f, \mathcal{U} f)$ whose restriction to each element $K$ is defined as the function in $\boldsymbol{V}(K) \times W(K)$ satisfying

$$
\begin{array}{cc}
(c \mathbf{Q} f, \boldsymbol{v})_{K}-(\mathcal{U} f, \operatorname{div} \boldsymbol{v})_{K}=0 & \text { for all } \boldsymbol{v} \in \boldsymbol{V}(K), \\
-(\operatorname{grad} w, \boldsymbol{Q} f)_{K}+\langle w, \widehat{\mathbf{Q}} f \cdot \boldsymbol{n}\rangle_{\partial K}+(d \mathcal{U} f, w)_{K}=(f, w)_{K} & \text { for all } w \in W(K) .
\end{array}
$$

Just as for the first local solver, we leave undefined the numerical trace $\widehat{\mathbf{Q}} f$. 
Obviously, while the functions $\left.(\mathbf{Q} f, \mathcal{U} f)\right|_{K}$ and $\left.(\mathbf{Q m}, \mathcal{U} \mathrm{m})\right|_{K}$ are in $\boldsymbol{V}(K) \times W(K)$, the space in which $\widehat{\mathbf{Q}} f$ and $\widehat{\mathbf{Q}} \mathrm{m}$ lie will vary from example to example. Now we make our assumption about the local solvers.

Assumption 2.1 (Existence and uniqueness of the local solvers). For every $\mathrm{m}$ in $\mathrm{M}_{h}$, there is a unique set of functions of $\mathrm{m},(\mathbf{Q} \mathrm{m}, \widehat{\mathbf{Q}} \mathrm{m}, \mathcal{U} \mathrm{m})$, depending linearly on $\mathrm{m}$, and satisfying (2.3). Furthermore, for every $f$ in $L^{2}(\Omega)$ there is a unique set of functions $(\mathbf{Q} f, \widehat{\mathbf{Q}} f, \mathcal{U} f)$, depending linearly on $f$, and satisfying (2.4).

Each of the methods under consideration define an approximation to $(\boldsymbol{q}, u)$,

$$
\left(\boldsymbol{q}_{h}, u_{h}\right)=\left(\mathbf{Q} \lambda_{h}+\mathbf{Q} g_{h}+\mathbf{Q} f, \mathcal{U} \lambda_{h}+\mathcal{U} g_{h}+\mathcal{U} f\right) \in\left(\boldsymbol{V}_{h} \times W_{h}\right),
$$

where $\lambda_{h}$ is assumed to be determined by the following discrete version of the transmission condition (1.5):

$$
\left\langle\mu, \llbracket \widehat{\mathbf{Q}} \lambda_{h}+\widehat{\mathbf{Q}} g_{h}+\widehat{\mathbf{Q}} f \rrbracket\right\rangle_{\varepsilon_{h}}=0 \quad \text { for all } \mu \in M_{h} .
$$

If we define the numerical flux by

$$
\widehat{\boldsymbol{q}}_{h}:=\widehat{\mathbf{Q}} \lambda_{h}+\widehat{\mathbf{Q}} g_{h}+\widehat{\mathbf{Q}} f,
$$

and if the (extension by zero to $\mathcal{E}_{h}$ of the) function $\left.\llbracket \widehat{\boldsymbol{q}}_{h} \rrbracket\right|_{\mathcal{E}_{h}}$ belongs to the space $M_{h}$, then the condition (2.6) is simply stating that $\left.\llbracket \widehat{\boldsymbol{q}}_{h} \rrbracket\right|_{\varepsilon_{h}^{\circ}}=0$ pointwise, that is, the normal component of the numerical trace $\widehat{\boldsymbol{q}}_{h}$ is single valued, or, adopting the terminology of [5], the function $\widehat{\boldsymbol{q}}_{h}$ is a conservative numerical flux. It is for this reason we call (2.6) the conservativity condition. If the function $\left.\llbracket \widehat{\boldsymbol{q}}_{h} \rrbracket\right|_{\mathcal{E}_{h}^{\circ}}$ does not belong to the space $M_{h}$, the conservativity condition imposes only the weak continuity of the normal component of the numerical trace $\widehat{\boldsymbol{q}}_{h}$ which, as a consequence, is not single valued.

It is worth noting that the method just described can be viewed as seeking the approximation $\left(\boldsymbol{q}_{h}, u_{h}, \lambda_{h}\right)$ in $\boldsymbol{V}_{h} \times W_{h} \times M_{h}$ satisfying

$$
\begin{array}{rlrl}
\left(c \boldsymbol{q}_{h}, \boldsymbol{r}\right)_{\mathcal{T}_{h}}-\left(u_{h}, \operatorname{div} \boldsymbol{r}\right)_{\mathcal{T}_{h}}+\sum_{K \in \mathcal{T}_{h}}\left\langle\lambda_{h}, \boldsymbol{r} \cdot \boldsymbol{n}\right\rangle_{\partial K \backslash \partial \Omega} & =-\left\langle g_{h}, \boldsymbol{r} \cdot \boldsymbol{n}\right\rangle_{\partial \Omega} & & \text { for all } \boldsymbol{r} \in \boldsymbol{V}_{h}, \\
-\left(\boldsymbol{q}_{h}, \operatorname{grad} w\right)_{\mathcal{T}_{h}}+\sum_{K \in \mathcal{T}_{h}}\left\langle\widehat{\boldsymbol{q}}_{h} \cdot \boldsymbol{n}, w\right\rangle_{\partial K}+\left(d u_{h}, w\right)_{\mathcal{T}_{h}} & =(f, w)_{\mathcal{T}_{h}} & \text { for all } w \in W_{h}, \\
\sum_{K \in \mathcal{T}_{h}}\left\langle\mu, \widehat{\boldsymbol{q}}_{h} \cdot \boldsymbol{n}\right\rangle_{\partial K}=0 & \text { for all } \mu \in M_{h} .
\end{array}
$$

Note that the first two equations are used to define the local solvers (2.3) and (2.4), while the last is nothing but the conservativity condition (2.6). This type of method is sometimes called a hybrid dual-mixed method. As pointed out in the introduction, it is called mixed because we seek approximations for the flux $\boldsymbol{q}_{h}$, as well as the potential $u_{h}$, on $\Omega$. It is called hybrid dual because the approximate trace, $\lambda_{h}$, associated to the conservativity condition is an approximation for the trace of the potential $u$ on the boundaries of the elements.

Many hybridized finite element methods admit this structure. For example, some classic hybridized mixed methods $[4,26]$ are obtained by an appropriate choice of the local spaces and by choosing $\widehat{\mathbf{Q}}(\cdot)$ in such a way that we have $\widehat{\boldsymbol{q}}_{h}=\boldsymbol{q}_{h}$. Many DG methods also fall into this form- although not all of them are hybridizable. Indeed, the schemes considered in the unified analysis of DG methods in [5] can be written in our notation as

$$
\begin{aligned}
& \quad\left(c \boldsymbol{q}_{h}, \boldsymbol{v}\right)_{\mathcal{T}_{h}}-\sum_{K \in \mathcal{T}_{h}}\left(u_{h}, \operatorname{div} \boldsymbol{v}\right)_{K}+\sum_{K \in \mathcal{T}_{h}}\left\langle\widehat{u}_{h}, \boldsymbol{v} \cdot \boldsymbol{n}\right\rangle_{\partial K \backslash \partial \Omega}=-\left\langle g_{h}, \boldsymbol{v} \cdot \boldsymbol{n}\right\rangle_{\partial \Omega}, \\
& -\left(\operatorname{grad} w, \boldsymbol{q}_{h}\right)_{\mathcal{T}_{h}}+\sum_{K \in \mathcal{T}_{h}}\left\langle w, \widehat{\boldsymbol{q}}_{h} \cdot \boldsymbol{n}\right\rangle_{\partial K}+\left(d u_{h}, w\right)_{\mathcal{T}_{h}}=(f, w)_{\mathcal{T}_{h}},
\end{aligned}
$$

where $\widehat{u}_{h}$ and $\widehat{\boldsymbol{q}}_{h}$ are the so called numerical traces of the DG method. Comparing these equations with the equations (2.8) of our general framework, we immediately realize that $\widehat{u}_{h}=\lambda_{h}$ on $\varepsilon_{h}^{\circ}$. We thus see that, for a finite element method to be hybridizable, its numerical trace $\widehat{u}_{h}$ must be single valued. This implies, in particular, that the DG methods in [5] that are not adjoint consistent cannot be hybridized by using our technique. In contrast, the (normal component of the) numerical trace $\widehat{\boldsymbol{q}}_{h}$ is not required to be single valued 
since the conservativity condition (2.6) does not always ensure a single-valued numerical trace. Thanks to this flexibility, the continuous Galerkin method and the embedded discontinuous Galerkin methods turn out to be hybridizable.

This concludes the description of the general structure of the methods. Methods with this structure include a wide class of DG and hybridized mixed and CG methods, as we show in Sections 3, 4 and 5.

2.3. The characterization of the variable $\lambda_{h}$. As we see next, the relevance of the methods fitting the previously described general structure resides in the fact that the $\lambda_{h}$ can be characterized in terms of a simple weak formulation in which none of the other variables appear.

THEOREM 2.1. Suppose Assumption 2.1 on the existence and uniqueness of the local solvers holds. Then $\lambda_{h} \in M_{h}$ satisfies the conservativity condition (2.6) if and only if it satisfies

$$
a_{h}\left(\lambda_{h}, \mu\right)=b_{h}(\mu) \quad \text { for all } \mu \in M_{h},
$$

where

$$
\begin{aligned}
a_{h}(\eta, \mu)=(c \mathbf{Q} \eta, \mathbf{Q} \mu)_{\mathcal{T}_{h}}+(d \mathcal{U} \eta, \mathcal{U} \mu)_{\mathcal{T}_{h}} & +\langle 1, \llbracket(\mathcal{U}-\mu)(\widehat{\mathbf{Q}} \eta-\mathbf{Q} \eta) \rrbracket\rangle_{\mathcal{E}_{h}}, \\
b_{h}(\mu)=\left\langle g_{h}, \llbracket \widehat{\mathbf{Q}} \mu \rrbracket\right\rangle_{\mathcal{E}_{h}}+(f, \mathcal{U} \mu)_{\mathcal{T}_{h}} & -\langle 1, \llbracket(\mathcal{U}-\mu)(\widehat{\mathbf{Q}} f-\mathbf{Q} f) \rrbracket\rangle_{\mathcal{E}_{h}} \\
& +\langle 1, \llbracket \mathcal{U}(\widehat{\mathbf{Q}} \mu-\mathbf{Q} \mu) \rrbracket\rangle_{\mathcal{E}_{h}} \\
& -\left\langle 1, \llbracket(\mathcal{U} \mu-\mu)\left(\widehat{\mathbf{Q}} g_{h}-\mathbf{Q} g_{h}\right) \rrbracket\right\rangle_{\mathcal{E}_{h}} \\
& +\left\langle 1, \llbracket\left(\mathcal{U} g_{h}-g\right)(\widehat{\mathbf{Q}} \mu-\mathbf{Q} \mu) \rrbracket\right\rangle_{\mathcal{E}_{h}},
\end{aligned}
$$

for all $\eta$ and $\mu \in M_{h}$.

Note that, since $\lambda_{h}$ is an approximation of the function $u$ on $\mathcal{E}_{h}^{\circ}$, it is natural to expect the bilinear form $a_{h}(\cdot, \cdot)$ to be symmetric. This motivates the following observation. The bilinear form $a_{h}(\cdot, \cdot)$ is symmetric if and only if the numerical trace $\widehat{\mathbf{Q}}$. is such that

$$
\left\langle 1, \llbracket(\mathcal{U} \mu-\mu)\left(\widehat{\mathbf{Q}}_{\eta}-\mathbf{Q} \eta\right) \rrbracket\right\rangle_{\varepsilon_{h}}=\left\langle 1, \llbracket\left(\mathcal{U}_{\eta}-\eta\right)\left(\widehat{\mathbf{Q}}_{\mu}-\mathbf{Q} \mu\right) \rrbracket\right\rangle_{\varepsilon_{h}}
$$

for all $\eta, \mu \in M_{h}$. If we also have

$$
\langle 1, \llbracket(\mathcal{U} \mu-\mu)(\widehat{\mathbf{Q}} f-\mathbf{Q} f) \rrbracket\rangle_{\varepsilon_{h}}=\langle 1, \llbracket \mathcal{U} f(\widehat{\mathbf{Q}} \mu-\mathbf{Q} \mu) \rrbracket\rangle_{\varepsilon_{h}},
$$

then

$$
b_{h}(\mu)=\left\langle g_{h}, \llbracket \widehat{\mathbf{Q}} \mu \rrbracket\right\rangle_{\mathcal{E}_{h}}+(f, \mathcal{U} \mu)_{\Omega} .
$$

All the examples in this paper satisfy the above symmetry conditions.

Now we prove Theorem 2.1. Set

$$
\begin{aligned}
a_{h}\left(\lambda_{h}, \mu\right) & =-\left\langle\mu, \llbracket \widehat{\mathbf{Q}} \lambda_{h} \rrbracket\right\rangle_{\mathcal{E}_{h}}, \\
b_{h}(\mu) & =\left\langle\mu, \llbracket \widehat{\mathbf{Q}} g_{h}+\widehat{\mathbf{Q}} f \rrbracket\right\rangle_{\mathcal{E}_{h}},
\end{aligned}
$$

so that the conservativity condition (2.6) takes the form (2.9). Theorem 2.1 then follows from the following result.

LEMMA 2.2 (Elementary identities). We have, for any $\mathrm{m}, \mu \in \mathrm{M}_{h}$ and $f \in L^{2}(\Omega)$,

$$
\begin{aligned}
-\langle\mu, \llbracket \widehat{\mathbf{Q}} \mathrm{m} \rrbracket\rangle_{\mathcal{E}_{h}}= & (c \mathbf{Q} \mathrm{m}, \mathbf{Q} \mu)_{\Omega}+(d \mathcal{U} \mathrm{m}, \mathcal{U} \mu)_{\Omega} \\
& +\langle 1, \llbracket(\mathcal{U} \mu-\mu)(\widehat{\mathbf{Q}} \mathrm{m}-\mathbf{Q} \mathrm{m}) \rrbracket\rangle_{\mathcal{E}_{h}}, \\
-\left\langle\mu, \llbracket \widehat{\mathbf{Q}} g_{h} \rrbracket\right\rangle_{\mathcal{E}_{h}}= & -\left\langle g_{h}, \llbracket \widehat{\mathbf{Q}} \mu \rrbracket\right\rangle_{\mathcal{E}_{h}} \\
& +\left\langle 1, \llbracket(\mathcal{U} \mu-\mu)\left(\widehat{\mathbf{Q}} g_{h}-\mathbf{Q}_{g_{h}}\right) \rrbracket\right\rangle_{\mathcal{E}_{h}} \\
& -\left\langle 1, \llbracket\left(\mathcal{U} g_{h}-g_{h}\right)(\widehat{\mathbf{Q}} \mu-\mathbf{Q} \mu) \rrbracket\right\rangle_{\mathcal{E}_{h}}, \\
-\langle\mu, \llbracket \widehat{\mathbf{Q}} f \rrbracket\rangle_{\mathcal{E}_{h}}= & -(f, \mathcal{U} \mu)_{\mathcal{T}_{h}} \\
& +\langle 1, \llbracket(\mathcal{U} \mu-\mu)(\widehat{\mathbf{Q}} f-\mathbf{Q} f) \rrbracket\rangle_{\mathcal{E}_{h}} \\
& -\langle 1, \llbracket \mathcal{U} f(\widehat{\mathbf{Q}} \mu-\mathbf{Q} \mu) \rrbracket\rangle_{\mathcal{E}_{h}} .
\end{aligned}
$$


To prove Lemma 2.2, we need some identities which follow from the equations defining the local solvers by integration by parts.

LEMma 2.3 (Relation between jumps and local residuals). For any $\mathrm{m}, \mu \in \mathrm{M}_{h}, f \in L^{2}(\Omega), \boldsymbol{v} \in \boldsymbol{V}_{h}$, and $w \in W_{h}$, the following identities hold:

$$
\begin{aligned}
& (c \mathbf{Q m}+\operatorname{grad} \mathcal{U}, \boldsymbol{v})_{\mathcal{T}_{h}}=+\langle 1, \llbracket(\mathcal{U}-\mathrm{m}) \boldsymbol{v} \rrbracket\rangle_{\mathcal{E}_{h}}, \\
& (\operatorname{div} \mathbf{Q} \mathrm{m}+d \mathcal{U}, w)_{\mathcal{T}_{h}}=-\langle 1, \llbracket w(\widehat{\mathbf{Q}} \mathrm{m}-\mathbf{Q} \mathrm{m}) \rrbracket\rangle_{\mathcal{E}_{h}}, \\
& (c \mathbf{Q} f+\operatorname{grad} \mathcal{U} f, \boldsymbol{v})_{\mathcal{T}_{h}}=+\langle 1, \llbracket \mathcal{U} f \boldsymbol{v} \rrbracket\rangle_{\mathcal{E}_{h}}, \\
& (\operatorname{div} \mathbf{Q} f+d \mathcal{U} f-f, w)_{\mathcal{T}_{h}}=-\langle 1, \llbracket w(\widehat{\mathbf{Q}} f-\mathbf{Q} f) \rrbracket\rangle_{\mathcal{E}_{h}} .
\end{aligned}
$$

Using these identities we now prove Lemma 2.2.

Proof. Let us prove the identity $(i)$. We have

$$
\begin{aligned}
& -\langle\mu, \llbracket \widehat{\mathbf{Q}} \mathrm{m} \rrbracket\rangle_{\mathcal{E}_{h}}=-\langle\mu, \llbracket \mathbf{Q} \mathrm{m} \rrbracket\rangle_{\mathcal{E}_{h}}-\langle\mu, \llbracket(\widehat{\mathbf{Q}} \mathrm{m}-\mathbf{Q} \mathrm{m}) \rrbracket\rangle_{\varepsilon_{h}} \\
& =(c \mathbf{Q} \mu, \mathbf{Q m})_{\mathcal{T}_{h}}-\left(\mathcal{U} \mu, \operatorname{div} \mathbf{Q m}_{\mathcal{T}_{h}}\right. \\
& -\langle\mu, \llbracket(\widehat{\mathbf{Q}} \mathrm{m}-\mathbf{Q} \mathrm{m}) \rrbracket\rangle_{\mathcal{E}_{h}} \quad \text { by }(2.3 \mathrm{a}), \\
& =\left(c \mathcal{Q} \mu, \mathbf{Q m}_{\mathcal{T}_{h}}+(d \mathcal{U} \mathrm{m}, \mathcal{U} \mu)_{\mathcal{T}_{h}}\right. \\
& +\left\langle 1, \llbracket \mathcal{U} \mu\left(\widehat{\mathbf{Q}}_{\mathrm{m}}-\mathbf{Q} \mathrm{m}\right) \rrbracket\right\rangle_{\varepsilon_{h}} \\
& -\langle\mu, \llbracket(\widehat{\mathbf{Q}} \mathrm{m}-\mathbf{Q} \mathrm{m}) \rrbracket\rangle_{\varepsilon_{h}} \text {. }
\end{aligned}
$$

This proves the identity $(i)$.

Now we prove $(i i)$. To do that, note that, by $(i)$, the bilinear form

$$
B(\mathrm{~m}, \mu)=\langle\mu, \llbracket \widehat{\mathbf{Q}} \mathrm{m} \rrbracket\rangle_{\mathcal{E}_{h}}+\langle 1, \llbracket(\mathcal{U} \mu-\mu)(\widehat{\mathbf{Q}} \mathrm{m}-\mathbf{Q} \mathrm{m}) \rrbracket\rangle_{\mathcal{E}_{h}},
$$

is symmetric. As a consequence, $(i i)$ follows from the equality $B\left(\mu, g_{h}\right)=B\left(g_{h}, \mu\right)$.

Finally, we prove $(i i i)$. We have

$$
\begin{aligned}
& -\langle\mu, \llbracket \widehat{\mathbf{Q}} f \rrbracket\rangle_{\mathcal{E}_{h}}=-\langle\mu, \llbracket \mathbf{Q} f \rrbracket\rangle_{\mathcal{E}_{h}}-\langle\mu, \llbracket(\widehat{\mathbf{Q}} f-\mathbf{Q} f) \rrbracket\rangle_{\varepsilon_{h}} \\
& =(c \mathbf{Q} \mu, \mathbf{Q} f)_{\mathcal{T}_{h}}-(\mathcal{U} \mu, \operatorname{div} \mathbf{Q} f)_{\mathcal{T}_{h}} \\
& -\langle\mu, \llbracket(\widehat{\mathbf{Q}} f-\mathbf{Q} f) \rrbracket\rangle_{\mathcal{E}_{h}} \quad \text { by }(2.3 \mathrm{a}) \text {, } \\
& =-(f, \mathcal{U} \mu)_{\mathcal{T}_{h}}+(c \mathbf{Q} \mu, \mathbf{Q} f)_{\mathcal{T}_{h}}+(d \mathcal{U} \mu, \mathcal{U} f)_{\mathcal{T}_{h}} \\
& +\langle 1, \llbracket(\mathcal{U} \mu-\mu)(\widehat{\mathbf{Q}} f-\mathbf{Q} f) \rrbracket\rangle_{\mathcal{E}_{h}} \quad \text { by }(2.12 \mathrm{~d}) \text {, } \\
& =-(f, \mathcal{U} \mu)_{\mathcal{T}_{h}}+(\operatorname{div} \mathcal{Q} \mu, \mathcal{U} f)_{\mathcal{T}_{h}}+(d \mathcal{U} \mu, \mathcal{U} f)_{\mathcal{T}_{h}} \\
& +\langle 1, \llbracket(\mathcal{U} \mu-\mu)(\widehat{\mathbf{Q}} f-\mathbf{Q} f) \rrbracket\rangle_{\varepsilon_{h}}, \quad \text { by }(2.4 \mathrm{a}), \\
& =-(f, \mathcal{U} \mu)_{\mathcal{T}_{h}}-\langle 1, \llbracket \mathcal{U} f(\widehat{\mathbf{Q}} \mu-\mathbf{Q} \mu) \rrbracket\rangle_{\varepsilon_{h}} \\
& +\langle 1, \llbracket(\mathcal{U} \mu-\mu)(\widehat{\mathbf{Q}} f-\mathbf{Q} f) \rrbracket\rangle_{\varepsilon_{h}} \quad \text { by }(2.12 \mathrm{~b}) \text {. }
\end{aligned}
$$

This completes the proof of Lemma 2.2.

2.4. Sufficient conditions for the existence and uniqueness of $\lambda_{h}$. Next, we provide two conditions which are sufficient for the existence and uniqueness of $\lambda_{h}$. The first is a condition on the local solvers and the second a condition on the relation between the local solvers on each element $K$ of the triangulation $\mathcal{T}_{h}$ and the global space $\mathrm{M}_{h}$ of approximate traces. It is worth emphasizing that, by guaranteeing the existence and uniqueness of $\lambda_{h}$, these simple conditions ensure the automatic coupling of the different local solvers even across non-matching meshes. Note that no explicit conditions on the triangulation $\mathcal{T}_{h}$ are involved in these conditions. 
Assumption 2.2 (on the positive semidefiniteness of the local solvers). The local solvers and the numerical flux traces in (2.3) and (2.4) are such that for every $K \in \mathcal{T}_{h}$, the following holds:

$$
-\langle\mu, \widehat{\mathbf{Q}} \mu \cdot \boldsymbol{n}\rangle_{\partial K} \geq 0 \quad \text { for all } \mu \in \mathrm{M}_{h} \text {. }
$$

Moreover, there exits a space $M(\partial K)$ containing the set $\left\{\nu:\left.\nu\right|_{e} \in \mathcal{P}_{0}(e)\right.$ on each face $e \in \mathcal{E}_{h}^{\circ}$ lying on $\left.\partial K\right\}$ such that

$$
\text { if }\langle\mu, \widehat{\mathbf{Q}} \mu \cdot \boldsymbol{n}\rangle_{\partial K}=0 \text { for some } \mu \in \mathrm{M}_{h} \text {, then } P_{\partial K} \mu=C_{K},
$$

for some constant $C_{K}$, where $P_{\partial K}$ is the $L^{2}(\partial K)$-orthogonal projection onto $M(\partial K)$.

Note that the auxiliary space $M(\partial K)$ is not necessarily finite dimensional. Its use is only theoretical; it is not used in practice in any way.

Let us argue that (2.13) is a reasonable condition on the positive semidefiniteness of the bilinear forms corresponding to the local solvers. Indeed, taking $\boldsymbol{v}:=\mathbf{Q} \mu$ in (2.3a), $\mathrm{m}:=\mu$ and $w:=\mathcal{U} \mathrm{m}$ in (2.3b), and adding the equations, we get,

$$
\begin{aligned}
-\langle\mathrm{m}, \widehat{\mathbf{Q}} \mu \cdot \boldsymbol{n}\rangle_{\partial K} & =(c \mathbf{Q m}, \mathbf{Q} \mu)_{K}+(d \mathfrak{U} \mathrm{m}, \mathfrak{U} \mu)_{K}+\langle(\widehat{\mathbf{Q}} \mathrm{m}-\mathbf{Q m}) \cdot \boldsymbol{n}, \mathfrak{U} \mu-\mu\rangle_{\partial K} \\
& =: a_{h, K}(\mathrm{~m}, \mu) .
\end{aligned}
$$

Thus, (2.13a) ensures that the bilinear form $a_{h, K}(\cdot, \cdot)$, which coincides with the form $a_{h}(\cdot, \cdot)$ when $\Omega$ is single element $K$, is positive semidefinite. Further, the condition (2.13b) states that those functions $\mathrm{m} \in \mathrm{M}_{h}$ for which $a_{h, K}(\mathrm{~m}, \mathrm{~m})=0$ yield constants under an appropriate projection. This is a reasonable assumption since it is a discrete version of a similar property of the exact solution. Indeed, for the exact solution, such a condition readily implies that $\mathbf{Q m}=0$ and, by the equations (1.4a), that $\mathrm{m}=\mathcal{U} \mathrm{m}=$ constant on $\partial K$.

This argument suggests that it is reasonable to expect the projection $P_{\partial K}$ to be strongly related to the identity, at least in parts of $\partial K$. The following assumption captures this property. It will allow us to establish a link between the different local solvers and, in so doing, to ensure the uniqueness of the solution of (1.7).

Assumption 2.3 (the "gluing condition"). If $\mu \in \mathrm{M}_{h}$ then, on every interior face $e=\partial K^{+} \cap \partial K^{-}$, either $\mu=P_{\partial K^{+}} \mu$ or $\mu=P_{\partial K^{-}} \mu$.

We are now ready to state our result.

TheOREM 2.4 (Existence and uniqueness of $\lambda_{h}$ ). If the Assumption 2.1 on the existence and uniqueness of the local solvers, Assumption 2.2 on the positive semidefiniteness of the local solvers, and Assumption 2.3, the gluing condition, hold, then there is a unique solution $\lambda_{h}$ of the weak formulation (2.9).

Proof. By Theorem 2.1, Assumption 2.1 guarantees the existence and uniqueness of $\widehat{\mathbf{Q}} \lambda_{h}$. Therefore the system (2.9) is well defined. Since it is a square system, to prove the existence and uniqueness of its solution, it is enough to show that if $a_{h}(\mu, \mu)=0$ for some $\mu \in M_{h}$, we have that $\mu=0$.

By Lemma 2.2,

$$
a_{h}(\mu, \mu)=-\langle\mu, \llbracket \widehat{\mathbb{Q}} \mu \rrbracket\rangle_{\varepsilon_{h}}=-\sum_{K \in \mathcal{T}_{h}}\langle\mu, \widehat{\mathbf{Q}} \mu \cdot \boldsymbol{n}\rangle_{\partial K} .
$$

Now since $a_{h}(\mu, \mu)=0$, by (2.13a) of Assumption 2.2 on on the positive semidefiniteness of the local solvers, each of the summands on the right hand side must vanish. Thus,

$$
\left\langle\mu, \widehat{\mathbf{Q}}_{\mu} \cdot \boldsymbol{n}\right\rangle_{\partial K}=0 \quad \text { for all } K \in \mathcal{T}_{h} .
$$

By the condition (2.13b), on any interior face $e=\partial K^{+} \cap \partial K^{-}$, this implies

$$
C_{K^{+}}=P_{\partial K^{+}} \mu=\frac{1}{|e|}\langle\mu, 1\rangle_{e}=P_{\partial K^{-}} \mu=C_{K^{-}},
$$

and by Assumption 2.3 (the gluing condition), we conclude that $C_{K^{+}}=\mu=C_{K^{-}}$on the face $e$. This means that $\mu$ is a constant on $\mathcal{E}_{h}$. Since $\mu=0$ on $\partial \Omega$, we see that $\mu$ is identically equal to zero on $\mathcal{E}_{h}$. This completes the proof. 


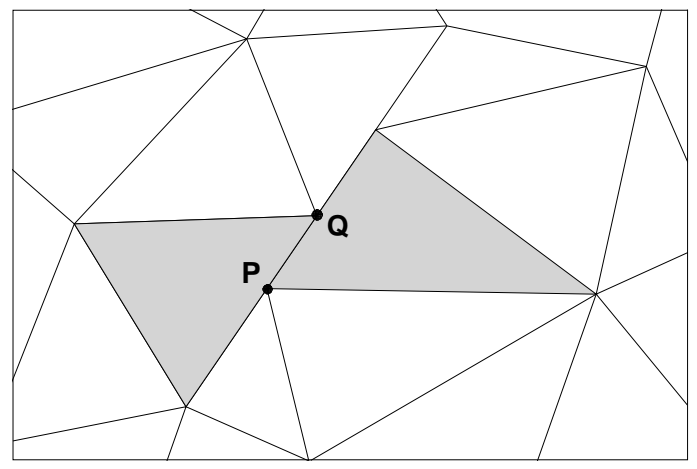

FIG. 2.1. The interior edge $e=\mathbf{P Q}$ and the support of the local solver $(\mathbf{Q m}, \mathbf{U m})$ for any $\mathrm{m}$ supported on e. The numerical trace $(\widehat{\mathbf{Q}} \mathrm{m})_{K}$ is generally nontrivial on the boundary of the two shadowed triangles $K$, but it vanishes on the boundary of other triangles.

2.5. The sparsity structure of the stiffness matrix for $\lambda_{h}$. Next, we comment on the sparsity structure of the stiffness matrix associated with the weak formulation (1.7). For any given basis of the space of approximate traces $M_{h}$, we denote by $[\mu]$ the corresponding vector of coefficients of the representation of $\mu$ in a given basis of $M_{h}$. Then the weak formulation (2.9)

$$
A\left[\lambda_{h}\right]=b,
$$

where

$$
[\mu]^{t} A\left[\lambda_{h}\right]=a_{h}\left(\lambda_{h}, \mu\right) \quad \text { and } \quad[\mu]^{t} b=b_{h}(\mu)
$$

Now, by the equations (2.11),

$$
a_{h}(\eta, \mu)=-\sum_{K \in \mathcal{T}_{h}}\langle\mu, \widehat{\mathbf{Q}} \eta \cdot \boldsymbol{n}\rangle_{\partial K} \quad \text { and } \quad b_{h}(\mu)=\sum_{K \in \mathcal{T}_{h}}\left\langle\mu,\left(\widehat{\mathbf{Q}} f+\widehat{\mathbf{Q}} g_{h}\right) \cdot \boldsymbol{n}\right\rangle_{\partial K}
$$

we have that

$$
A=\sum_{K \in \mathcal{T}_{h}} A_{K} \quad \text { and } \quad b=\sum_{K \in \mathcal{T}_{h}} b_{K}
$$

where $A_{K}$ and $b_{K}$ are defined by

$$
[\mu]^{t} A_{K}[\eta]=-\langle\mu, \widehat{\mathbf{Q}} \eta \cdot \boldsymbol{n}\rangle_{\partial K} \quad \text { and } \quad[\mu]^{t} b_{K}=\left\langle\mu,\left(\widehat{\mathbf{Q}} f+\widehat{\mathbf{Q}} g_{h}\right) \cdot \boldsymbol{n}\right\rangle_{\partial K} .
$$

Thus, the matrix equations for the multiplier can be obtained in a typical finite element manner. Moreover, the sparsity of the matrices $A_{K}$ and $b_{K}$ can be deduced from the following result.

Proposition 2.1. Suppose Assumption 2.1 on the existence and uniqueness of the local solvers holds. Then

(i) If the support of $\mu \in M_{h}$ does not intersect $\partial K$, we have that $[\mu]^{t} b_{K}=0$;

(ii) If the support of $\mu \in M_{h}$ or the support of $\eta \in M_{h}$ does not intersect $\partial K$, we have that $[\mu]^{t} A_{K}[\eta]=0$.

Proof. That $[\mu]^{t} b_{K}=0$ and $[\mu]^{t} A_{K}[\eta]=0$ if the support of $\mu$ does not intersect $\partial K$ follows immediately from the definition of $b_{K}$ and $A_{K}$. Let us show that $[\mu]^{t} A_{K}[\eta]=0$ if the support of $\eta$ does not intersect $\partial K$. Since we are assuming that the local solvers are well defined, if the support of $\eta$ does not intersect $\partial K$, we have, by Assumption 2.1, that $(\widehat{\mathbf{Q}} \eta)_{K}=0$ on $\partial K$ and the result follows. This completes the proof.

We emphasize that this result, illustrated in Fig. 2.1, is possible due to the fact that the numerical trace $\widehat{\mathbf{Q}}$. is double valued on all interior faces $e \in \mathcal{E}_{h}^{\circ}$. Indeed, take $\eta$ as in the above proof and further assume that its support intersects $\partial K^{\prime}$, where the intersection of $\partial K$ and $\partial K^{\prime}$ is a face $e$ in $\mathcal{E}_{h}^{\circ}$. Then $(\widehat{\mathbf{Q}} \eta)_{K^{\prime}}$ can be nontrivial on $e$, in general. However, this does not contradict the fact that $(\widehat{\mathbf{Q}} \eta)_{K}=0$ on $e$ because the function $\widehat{\mathbf{Q}} \eta$ is double valued on $e$. 
In the remainder of this subsection, we compare the number of globally coupled degrees of freedom and the number of non-zero entries of the stiffness matrix, restricting our attention to the case of a conforming triangulation $\mathcal{T}_{h}$ (no hanging nodes). First, consider the case in which $\mathrm{M}_{h}:=\mathcal{M}_{h, k}^{c}$, where

$$
\mathcal{M}_{h, k}^{c}:=\left\{\mu \in \mathcal{C}\left(\mathcal{E}_{h}\right):\left.\mu\right|_{e} \in \mathcal{P}_{k}(e) \text { for all faces } e \in \mathcal{E}_{h}\right\} .
$$

Here $\mathcal{C}\left(\mathcal{E}_{h}\right)$ denotes the space of continuous functions on $\mathcal{E}_{h}$, and $\mathcal{P}_{k}(D)$ the set of polynomials of degree at most $k$ on a domain $D$. Then the sparsity structure of the matrix $A$ is exactly that of the statically condensed stiffness matrix of a continuous Galerkin method using approximations whose restriction to each simplex $K$ is in $\mathcal{P}_{k}(K)$.

If instead we take $\mathrm{M}_{h}:=\mathcal{M}_{h, k}$, where

$$
\mathcal{M}_{h, k}=\left\{\mu \in L^{2}\left(\mathcal{E}_{h}\right):\left.\mu\right|_{e} \in \mathcal{P}_{k}(e) \text { for all faces } e \in \mathcal{E}_{h}^{\circ}\right\},
$$

then by choosing basis functions whose support is always contained in a single face, we obtain a matrix $A$ which has a block structure with square blocks of order equal to the dimension of $\mathcal{P}_{k}(e)$. The number of block rows and block columns is equal to the number of interior faces of the triangulation, $N_{\text {i.f. }}$, and, on each block row, there are at most $(2 n+1)$ blocks that are not equal to zero. In other words, the size and sparsity structure of the matrix $A$ is precisely that of the stiffness matrix for the hybridized RT method using $M_{h}$ as space of approximate traces; see [26]. This means that the order of the matrix $A$, which is equal to the number of degrees of freedom of $\lambda_{h}$, is given by

$$
N_{\text {d.o.f. }}=N_{\text {i.f. }} \operatorname{dim} \mathcal{P}_{k}(e)
$$

and that the number of possibly nonvanishing entries of $A$ is bounded by

$$
N_{\text {sparsity }}=N_{\text {i.f. }}(2 n+1)\left(\operatorname{dim} \mathcal{P}_{k}(e)\right)^{2} .
$$

Let us now compare the size and sparsity structure of this stiffness matrix with those of the IP and the (Schur-complement matrix of the) LDG methods that use polynomials of degree $k$. The number of globally coupled degrees of freedom for both methods is

$$
N_{\text {d.o.f. }}^{\mathrm{IP}}=N_{\text {d.o.f. }}^{\mathrm{LDG}}=N_{s} \operatorname{dim} \mathcal{P}_{k}(K),
$$

where $N_{s}$ denotes the number of simplexes of the triangulation. Moreover, the stiffness matrices in question have a block structure with square blocks of order equal to the dimension of $\mathcal{P}_{k}(K)$. On each block-row, the number of blocks that are not equal to zero are at most $(n+2)$ for the IP method and $\left((n+1)^{2}+1\right)$ for the LDG method; recall that for the LDG method, the degrees of freedom of the neighbors of the neighbors are involved. This means that the number of nonzero entries of the corresponding stiffness matrices are (bounded by)

$$
N_{\text {sparsity }}^{\mathrm{IP}}=N_{s}(n+2)\left(\operatorname{dim} \mathcal{P}_{k}(K)\right)^{2}, \quad N_{\text {sparsity }}^{\mathrm{LDG}}=N_{s}\left((n+1)^{2}+1\right)\left(\operatorname{dim} \mathcal{P}_{k}(K)\right)^{2} .
$$

To compare with the hybridized methods, we consider the ratio of the number of globally coupled degrees of freedom, $R_{\text {d.o.f. }}:=N_{\text {d.o.f. }}^{\text {DG }} / N_{\text {d.o.f. }}$ and the ratio of the number of entries different from zero, $R_{\text {sparsity }}^{\mathrm{IP}}:=$ $N_{\text {sparsity }}^{\mathrm{IP}} / N_{\text {sparsity }}$ and $R_{\text {sparsity }}^{\mathrm{LDG}}:=N_{\text {sparsity }}^{\mathrm{LDG}} / N_{\text {sparsity. }}$. Since $N_{s} / N_{\text {i.f. }} \approx 2 /(n+1)$ (up to a lower order term related to the faces on the boundary) then

$$
R_{\mathrm{sparsity}}^{\mathrm{IP}}=\frac{2(n+2)}{(n+1)(2 n+1)}\left(\frac{k}{n}+1\right)^{2}, \quad R_{\mathrm{sparsity}}^{\mathrm{LDG}}=\frac{2\left((n+1)^{2}+1\right)}{(n+1)(2 n+1)}\left(\frac{k}{n}+1\right)^{2} .
$$

In Table 2.1, we see that in two- or three-space dimensions, the hybridizable methods always have less degrees of freedom and have a stiffness matrix that is sparser than the corresponding LDG methods. The same is valid for the IP method in two-space dimensions, and in three-space dimensions for $k \geq 3$. In three-space dimensions, the IP method with $k=1$ is more advantageous than the corresponding hybridizable DG method; for $k=2$ its advantages are, however, marginal. 
TABLE 2.1

Comparison between hybridizable DG methods and two typical DG methods on simplicial meshes.

\begin{tabular}{|c|c|c|c|c|c|c|c|c|c|}
\hline \multirow[t]{2}{*}{$n$} & \multirow[t]{2}{*}{$k$} & \multirow[t]{2}{*}{$R_{\text {d.o.f. }}$} & \multicolumn{2}{|c|}{$R_{\text {sparsity }}$} & \multirow[t]{2}{*}{$n$} & \multirow[t]{2}{*}{$k$} & \multirow[t]{2}{*}{$R_{\text {d.o.f. }}$} & \multicolumn{2}{|c|}{$R_{\text {sparsity }}$} \\
\hline & & & IP & LDG & & & & IP & LDG \\
\hline \multirow[t]{4}{*}{2} & 1 & 1.00 & 1.20 & 3.00 & 3 & 1 & 0.67 & 0.63 & 2.16 \\
\hline & 2 & 1.33 & 2.13 & 5.33 & & 2 & 0.83 & 0.99 & 3.37 \\
\hline & 3 & 1.67 & 3.33 & 8.33 & & 3 & 1.00 & 1.42 & 4.86 \\
\hline & 4 & 2.00 & 4.80 & 12.00 & & 4 & 1.17 & 1.94 & 6.61 \\
\hline
\end{tabular}

TABLE 2.2

Comparison between hybridizable and the statically-condensed IP methods on simplicial meshes.

\begin{tabular}{cccccccc}
\hline$n$ & $k$ & $R_{\text {d.o.f. }}$ & $R_{\text {sparsity }}$ & $n$ & $k$ & $R_{\text {d.o.f. }}$ & $R_{\text {sparsity }}$ \\
\hline \hline 2 & 3 & $\mathbf{1 . 5 0}$ & $\mathbf{2 . 7 0}$ & 3 & 4 & $\mathbf{1 . 1 3}$ & $\mathbf{1 . 8 6}$ \\
& 4 & $\mathbf{1 . 6 0}$ & $\mathbf{3 . 0 7}$ & & 5 & $\mathbf{1 . 2 3}$ & $\mathbf{2 . 1 9}$ \\
& 5 & $\mathbf{1 . 6 7}$ & $\mathbf{3 . 3 3}$ & & 6 & $\mathbf{1 . 3 2}$ & $\mathbf{2 . 4 9}$ \\
\hline
\end{tabular}

It is interesting to extend the comparison with the IP method for which static condensation of the interior degrees of freedom has been carried out; of course this can be done only if $k \geq n+1$. In this case, the number of globally coupled degrees of freedom is

$$
N_{\text {d.o.f. }}^{\text {sc-IP }}=N_{s}\left(\operatorname{dim} \mathcal{P}_{k}(K)-\operatorname{dim} \mathcal{P}_{k-n-1}(K)\right) .
$$

The stiffness matrix in question has again a block structure with square blocks of order equal to $\left(\operatorname{dim} \mathcal{P}_{k}(K)-\right.$ $\left.\operatorname{dim} \mathcal{P}_{k-n-1}(K)\right)$. On each block-row, the number of blocks that are not equal to zero are $n+2$. Indeed, it can be shown that the interior degrees of freedom on a given simplex can be expressed in terms of the condensed degrees of freedom of the simplex and those of its neighbors, and that the condensed degrees freedom can be expressed in terms of the interior degrees of freedom of the simplex and those of its neighbors. We then have

$$
N_{\text {sparsity }}^{\text {sc-IP }}=N_{s}(n+2)\left(\operatorname{dim} \mathcal{P}_{k}(K)-\operatorname{dim} \mathcal{P}_{k-n-1}(K)\right)^{2} .
$$

This implies that the corresponding ratios are

$$
R_{\text {d.o.f. }}^{\text {sc-IP }}=\frac{2}{(n+1)}\left(\frac{k}{n}+1\right)\left(1-\Pi_{j=1}^{n} \frac{k-j}{k+j}\right)
$$

and

$$
R_{\mathrm{sparsity}}^{\mathrm{sc}-\mathrm{IP}}=\frac{2(n+2)}{(n+1)(2 n+1)}\left(\frac{k}{n}+1\right)^{2}\left(1-\Pi_{j=1}^{n} \frac{k-j}{k+j}\right)^{2}
$$

We show some results in Table 2.2. We see that the hybridized methods produce smaller and more sparse matrices than the statically-condensed IP method.

The same argument could be made for DG methods on $n$-dimensional rectangular finite elements. In this case the DG approximations could be based on polynomials of degree $k$ (instead of polynomials of degree $k$ in each variable in the case of continuous elements). Then the ratio between the degrees of freedom (and the sparsity) will be lower, since instead of the factor $N_{s} / N_{\text {i.f. }} \approx 2 /(n+1)$ we have the factor $N_{r} / N_{\text {i.f. }} \approx 2 / 2^{n}$.

A complete comparison of methods would require factoring in the costs of solving the algebraic problem. While greater sparsity or lesser number of degrees of freedom often yields faster solution methods, definitive conclusions can be made only after numerical experiments with specific direct or iterative methods; see [16] for such studies on older methods. 
3. Examples of hybridizable methods. In this section, we give several examples of methods fitting the general structure described in the previous section. We restrict ourselves to methods that use the same local solver in all the elements $K$ of the triangulation $\mathcal{T}_{h}$. Throughout this section we assume that $\mathcal{T}_{h}$ is a conforming simplicial triangulation.

To define each of the methods, we only have to specify (1) the numerical trace of the flux $\widehat{\mathbf{Q}} \cdot,(2)$ the local spaces $\boldsymbol{V}(K), W(K)$, and (3) the space of approximate traces $M_{h}$. We then verify that the local solvers are well-posed and discuss the conservativity condition by using Theorem 2.1. We use Theorem 2.4 to verify the existence and uniqueness of the approximate trace $\lambda_{h}$, and end by relating these results to relevant, earlier material.

Our examples are summarized Tables 3.1 and 3.2; some of them are schematicaly related in Fig. 3.1. The first column of the tables consists of method names. We adopt the following convention: Suppose that we define the local solver on each element by using a numerical method previously known as the "N" method. Then we call the resulting hybridized formulation an "N-hybridizable method", or, in short, an "N-H" method. For example, if we use the well known IP method to define the local solvers, then any hybridized formulation with such local solvers is denoted as IP-H. We also say that a finite element method is an N-H method if there is a hybridization of the method that is an N-H method.

In columns 2-4 of Table 3.1 we give the spaces of the local solvers and the approximate trace. In the fifth column we indicate whether the method gives a single valued flux trace $\widehat{\boldsymbol{q}}_{h}$ so the conservativity condition is satisfied in a strong form or $\widehat{\boldsymbol{q}}_{h}$ is double valued so the methods leads to a weak conservativity condition. In the last two columns of Table 3.1 we define the numerical traces of the fluxes $\widehat{\mathbf{Q}} \mathrm{m}$ and $\widehat{\mathbf{Q}} f$. The weak formulations for the approximate traces obtained via Theorem 2.1 for each type of method are listed in Table 3.2.

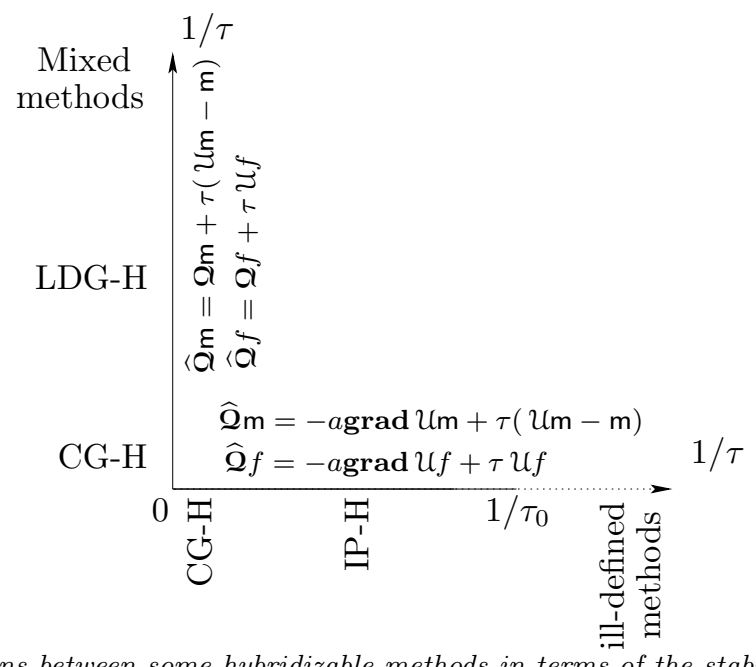

FIG. 3.1. Relations between some hybridizable methods in terms of the stabilization parameter $\tau$.

3.1. The RT-H method. This method is obtained by using the RT method to define the local solvers. The three ingredients of the RT-H method are as follows:

1. For each $K \in \mathcal{T}_{h}$, we take

$$
\widehat{\mathbf{Q}} \mathrm{m}=\mathbf{Q m}, \quad \widehat{\mathbf{Q}} f=\mathbf{Q} f \quad \text { on } \partial K ;
$$

2. The finite element space $\boldsymbol{V}(K) \times W(K)$ is defined as Raviart-Thomas space of degree $k$ :

$$
\boldsymbol{V}(K)=\mathcal{P}_{k}(K)^{n}+\boldsymbol{x} \mathcal{P}_{k}(K), \quad W(K)=\mathcal{P}_{k}(K), \quad k \geq 0,
$$

where $\mathcal{P}_{k}(K)^{n}$ denotes the set of vector functions whose components are in $\mathcal{P}_{k}(K)$;

3 . We define the space of approximate traces as:

$$
\mathrm{M}_{h}=\mathcal{M}_{h, k} .
$$


TABLE 3.1

Summary of the examples

\begin{tabular}{|c|c|c|c|c|c|c|}
\hline Method & $\boldsymbol{V}(K)$ & $W(K)$ & $\mathrm{M}_{h}$ & conservativity & $\widehat{\mathbf{Q}} \mathrm{m}$ & $\widehat{\mathbf{Q}} f$ \\
\hline RT-H & $\mathcal{P}_{k}(K)^{n}+\boldsymbol{x} \mathcal{P}_{k}(K)$ & $\mathcal{P}_{k}(K)$ & $\mathcal{M}_{h, k}$ & strong & $Q m$ & $\mathbf{Q} f$ \\
\hline BDM-H & $\mathcal{P}_{k}(K)^{n}$ & $\mathcal{P}_{k-1}(K)$ & $\mathcal{M}_{h, k}$ & strong & Qm & $\mathcal{Q} f$ \\
\hline LDG-H & $\mathcal{P}_{k}(K)^{n}$ & $\mathcal{P}_{k-1}(K)$ & $\mathcal{M}_{h, k}$ & strong & $\mathbf{Q} \mathrm{m}+\tau(\mathcal{U} \mathrm{m}-\mathrm{m}) \boldsymbol{n}$ & $\mathbf{Q} f+\tau(\mathcal{U} f) \boldsymbol{n}$ \\
\hline LDG-H & $\mathcal{P}_{k}(K)^{n}$ & $\mathcal{P}_{k}(K)$ & $\mathcal{M}_{h, k}$ & strong & $\mathbf{Q} \mathrm{m}+\tau(\mathcal{U} \mathrm{m}-\mathrm{m}) \boldsymbol{n}$ & $\boldsymbol{Q} f+\tau(\mathcal{U} f) \boldsymbol{n}$ \\
\hline LDG-H & $\mathcal{P}_{k-1}(K)^{n}$ & $\mathcal{P}_{k}(K)$ & $\mathcal{M}_{h, k}$ & strong & $\mathbf{Q m}+\tau(\mathcal{U} \mathrm{m}-\mathrm{m}) \boldsymbol{n}$ & $\mathbf{Q} f+\tau(\mathcal{U} f) \boldsymbol{n}$ \\
\hline $\mathrm{IP}-\mathrm{H}$ & $\mathcal{P}_{k}(K)^{n}$ & $\mathcal{P}_{k}(K)$ & $\mathcal{M}_{h, k}$ & strong & $-a \operatorname{grad} U \mathrm{~m}+\tau(\mathcal{U} \mathrm{m}-\mathrm{m}) \boldsymbol{n}$ & $-a \operatorname{grad} \mathcal{U} f+\tau(\mathcal{U} f) \boldsymbol{n}$ \\
\hline $\mathrm{NC}-\mathrm{H}$ & $\mathcal{P}_{k-1}(K)^{2}, k$ odd & $\mathcal{P}_{k}(K)$ & $\mathcal{M}_{h, k-1}$ & strong & a new unknown variable & a new unknown variable \\
\hline CG-H & $\mathcal{P}_{k-1}(K)^{n}$ & $\mathcal{P}_{k}(K)$ & $\mathcal{M}_{h, k}^{c}$ & weak & a new unknown variable & a new unknown variable \\
\hline
\end{tabular}

TABLE 3.2

Weak formulations for the approximate trace.

\begin{tabular}{|c|c|c|}
\hline Method & $a_{h}(\eta, \mu)$ & $b_{h}(\mu)$ \\
\hline RT-H & $(c \mathbf{Q} \eta, \mathbf{Q} \mu)_{\mathcal{T}_{h}}+(d \mathcal{U} \eta, \mathcal{U} \mu)_{\mathcal{T}_{h}}$ & $(f, \mathcal{U} \mu)_{\mathcal{T}_{h}}+\left\langle g_{h}, \boldsymbol{Q} \mu \cdot \boldsymbol{n}\right\rangle_{\partial \Omega}$ \\
\hline BDM-H & $\left(c \mathbf{Q}_{\eta}, \mathbf{Q} \mu\right)_{\mathcal{T}_{h}}+\left(d \mathcal{U} \eta, \mathcal{U}_{\mu}\right)_{\mathcal{T}_{h}}$ & $\left(f, \mathcal{U}_{\mu}\right)_{\mathcal{T}_{h}}+\left\langle g_{h}, \mathbf{Q}_{\mu} \cdot \boldsymbol{n}\right\rangle_{\partial \Omega}$ \\
\hline LDG-H & $\begin{array}{l}\left(c \mathbf{Q} \eta, \mathbf{Q}_{\mu}\right)_{\mathcal{T}_{h}}+\left(d \mathcal{U} \eta, \mathcal{U}_{\mu}\right)_{\mathcal{T}_{h}}+ \\
\quad\langle 1, \llbracket(\mathcal{U} \mu-\mu)(\tau(\mathcal{U} \eta-\eta) \boldsymbol{n}) \rrbracket\rangle_{\mathcal{E}_{h}}\end{array}$ & $(f, \mathcal{U} \mu)_{\mathcal{T}_{h}}+\left\langle g_{h}, \mathbf{Q}_{\mu} \cdot \boldsymbol{n}+\tau \mathcal{U} \mu\right\rangle_{\partial \Omega}$ \\
\hline $\mathrm{IP}-\mathrm{H}^{\dagger}$ & $\begin{array}{l}(a \operatorname{grad} \mathcal{U} \mu, \operatorname{grad} \mathcal{U} \eta)_{\mathcal{T}_{h}}+(d \mathcal{U} \eta, \mathcal{U} \mu)_{\mathcal{T}_{h}}+ \\
\quad\langle 1, \llbracket((\eta-\mathcal{U} \eta) a \operatorname{grad} \mathcal{U} \mu+(\mu-\mathcal{U} \mu) a \operatorname{grad} \mathcal{U} \eta) \rrbracket\rangle_{\mathcal{E}_{h}}+ \\
\quad\langle 1, \llbracket(\mathcal{U} \mu-\mu)(\tau(\mathcal{U} \eta-\eta) \boldsymbol{n}) \rrbracket\rangle_{\mathcal{E}_{h}}\end{array}$ & $(f, \mathcal{U} \mu)_{\mathcal{T}_{h}}+\left\langle g_{h},-a \operatorname{grad} \mathcal{U} \mu \cdot \boldsymbol{n}+\tau \mathcal{U} \mu\right\rangle_{\partial \Omega}$ \\
\hline $\mathrm{NC}-\mathrm{H}^{\dagger}$ & $(a \operatorname{grad} \mathcal{U} \eta, \operatorname{grad} \mathcal{U} \mu)_{\mathcal{T}_{h}}+(d \mathcal{U} \eta, \mathcal{U} \mu)_{\mathcal{T}_{h}}$ & $(f, \mathcal{U} \mu)_{\mathcal{T}_{h}}+\left\langle g_{h}, \widehat{\mathbf{Q}}_{\mu} \cdot \boldsymbol{n}\right\rangle_{\partial \Omega}$ \\
\hline $\mathrm{CG}-\mathrm{H}^{\dagger}$ & $\left(a \operatorname{grad} U \mathcal{U}_{\eta}, \operatorname{grad} \mathcal{U} \mu\right)_{\mathcal{T}_{h}}+(d \mathcal{U} \eta, \mathcal{U} \mu)_{\mathcal{T}_{h}}$ & $(f, \mathcal{U} \mu)_{\mathcal{T}_{h}}+\left\langle g_{h}, \llbracket \widehat{\mathbf{Q}} \mu \rrbracket\right\rangle_{\varepsilon_{h}}$ \\
\hline
\end{tabular}


The fact that the local solvers are well defined can be established by realizing that they are defined by using exactly the RT mixed finite element method. Indeed, if we insert the expression of the numerical traces $\widehat{\mathbf{Q}} \mathrm{m}$ and $\widehat{\mathbf{Q}} f$, into the equations defining the local solvers, we see that they are nothing but the RT discretizations of the exact local problems (1.4), as claimed. Since the RT method is well defined, see [49, 12], the local solvers $(\mathbf{Q m}, \mathcal{U m})$ and $(\mathbf{Q} f, \mathcal{U} f)$ are also well defined.

Note that the conservativity condition (2.6) forces the numerical trace $\widehat{\boldsymbol{q}}_{h}$ to be single-valued. Indeed, because the (extension by zero from $\mathcal{E}_{h}^{\circ}$ to $\mathcal{E}_{h}$ of) $\llbracket \widehat{\mathbf{Q}} \lambda_{h}+\widehat{\mathbf{Q}} g_{h}+\widehat{\mathbf{Q}} f \rrbracket$ and the test functions $\mu$ belong to the same space, the conservativity condition (2.6) forces the equality

$$
\llbracket \widehat{\boldsymbol{q}}_{h} \rrbracket=\llbracket \boldsymbol{q}_{h} \rrbracket=\llbracket \widehat{\mathbf{Q}} \lambda_{h}+\widehat{\mathbf{Q}} g_{h}+\widehat{\mathbf{Q}} f \rrbracket=0 \quad \text { on } \quad \mathcal{E}_{h}^{\circ},
$$

so the normal component of the numerical trace $\widehat{\boldsymbol{q}}_{h}$ is single valued and $\boldsymbol{q}_{h} \in H(\operatorname{div}, \Omega)$. Moreover, Theorem 2.1 asserts that the conservativity condition is equivalent to (2.9) with

$$
\begin{aligned}
a_{h}(\eta, \mu) & =(c \mathbf{Q} \eta, \mathbf{Q} \mu)_{\mathcal{T}_{h}}+(d \mathcal{U} \eta, \mathcal{U} \mu)_{\mathcal{T}_{h}}, \\
b_{h}(\mu) & =\left\langle g_{h}, \mathbf{Q} \mu \cdot \boldsymbol{n}\right\rangle_{\partial \Omega}+(f, \mathcal{U} \mu)_{\mathcal{T}_{h}},
\end{aligned}
$$

provided $\left.g_{h}\right|_{\mathcal{E}_{h}^{\circ}}=0$. This is, of course, a reasonable choice since $\left.g\right|_{\mathcal{E}_{h}^{\circ}}=0$ and $\mathrm{M}_{h}$ is a space of discontinuous functions.

These results appeared earlier in [26, Theorem 2.1], where the hybridized RT method of arbitrary order was considered; the case of the lowest order RT method was previously considered in [21]. We can thus conclude that the original RT method is an RT-H method. In [42], the bilinear form $a_{h}(\cdot, \cdot)$ was shown to be positive definite; this implies that $\lambda_{h}$ is uniquely determined. Next, we apply our general approach to this method and verify Assumption 2.2 on the positive semidefiniteness of the local solvers and Assumption 2.3, the gluing condition. By Theorem 2.4, this ensures the existence and uniqueness of $\lambda_{h}$ and hence that of the approximation $\left(\boldsymbol{q}_{h}, u_{h}\right)$.

Proposition 3.1. Assumption 2.1 on the existence and uniqueness of the local solvers, and Assumption 2.2 on the positive semidefiniteness of the local solvers hold for the RT-H method. Assumption 2.3, the gluing condition, also holds with

$$
M(\partial K)=\left\{\mu:\left.\mu\right|_{e} \in \mathcal{P}_{k}(e) \text { for all faces } e \text { of } \partial K\right\}
$$

Proof. The Assumption 2.1 obviously holds. Let us prove Assumption 2.2. To do that, we first show that the condition (2.13a) holds. By the identity (2.14) with $\mu:=\mathrm{m}$, we have that

$$
-\langle\mathrm{m}, \widehat{\mathbf{Q}} \mathrm{m} \cdot \boldsymbol{n}\rangle_{\partial K}=(c \mathbf{Q m}, \mathbf{Q m})_{K}+\left(d \mathcal{U}, \mathcal{U m}_{K},\right.
$$

by the definition of $\widehat{\mathbf{Q}} \mathrm{m}$. We thus see that the condition (2.13a) is satisfied.

Now we verify condition (2.13b) with the given choice of $M(\partial K)$. If $\langle\mathrm{m}, \widehat{\mathbf{Q}} \mathrm{m} \cdot \boldsymbol{n}\rangle_{\partial K}=0$, we immediately obtain $\left.\mathbf{Q m}\right|_{K}=0$. This implies that the equation (2.3a) can be rewritten as

$$
(\operatorname{grad} U \mathrm{~m}, \boldsymbol{v})_{K}-\langle\mathcal{U}-\mathrm{m}, \boldsymbol{v} \cdot \boldsymbol{n}\rangle_{\partial K}=0 \quad \text { for all } \boldsymbol{v} \in \boldsymbol{V}(K) .
$$

It is well known, see, for example, [12], that for a given grad $\mathcal{U} \mathrm{m}$ and $\mathcal{U} \mathrm{m}-\mathrm{m}$ there is a function $\boldsymbol{v} \in \boldsymbol{V}(K)$ such that

$$
\begin{aligned}
& \left(\boldsymbol{v}, \boldsymbol{p}_{k-1}\right)_{K}=\left(\operatorname{grad} U \mathrm{~m}, \boldsymbol{p}_{k-1}\right)_{K} \quad \text { for all } \boldsymbol{p}_{k-1} \in \mathcal{P}_{k-1}(K), \\
& \left\langle\boldsymbol{v} \cdot \boldsymbol{n}, p_{k}\right\rangle_{e}=-\left\langle\mathcal{U} \mathrm{m}-\mathrm{m}, p_{k}\right\rangle_{e} \quad \text { for all } p_{k} \in \mathcal{P}_{k}(e),
\end{aligned}
$$

for all faces $e$ of $K$. Using this $\boldsymbol{v}$ in (3.1), we find that

$$
(\operatorname{grad} U \mathrm{~m}, \operatorname{grad} U \mathrm{~m})_{K}+(\mathcal{U}-\mathrm{m}, \mathcal{U} \mathrm{m}-\mathrm{m})_{\partial K}=0 .
$$

This implies that $\mathcal{U} \mathrm{m}$ is a constant on $K$, so $\mathrm{m}$ is constant on $\partial K$. This proves that the condition (2.13b) is satisfied with $M(\partial K)$ as described.

It remains to verify Assumption 2.3. Since we are assuming that the triangulation $\mathcal{T}_{h}$ is conforming, each interior face $e=\partial K^{+} \cap \partial K^{-}$is also a face of both $K^{+}$and $K^{-}$. Hence, since $\left.\mu\right|_{e} \in \mathcal{P}_{k}(e)$, we have that $P_{\partial K^{+}} \mu=\mu=P_{\partial K^{-}} \mu$ on $e$. This completes the proof. 
3.2. The BDM-H method. To obtain the BDM-H method, we use the BDM method to define the main three ingredients of the hybridization method:

1. For each $K \in \mathcal{T}_{h}$ we take

$$
\widehat{\mathbf{Q}} \mathrm{m}=\mathbf{Q} \mathrm{m}, \quad \widehat{\mathbf{Q}} f=\mathbf{Q} f \quad \text { on } \partial K ;
$$

2. The finite element spaces are defined as

$$
\boldsymbol{V}(K)=\mathcal{P}_{k}(K)^{n}, \quad W(K)=\mathcal{P}_{k-1}(K), \quad k \geq 1 ;
$$

3. The space of approximate traces is defined as $\mathrm{M}_{h}=\mathcal{M}_{h, k}$.

This defines the BDM-H method.

Everything said about the RT-H method in the previous subsection applies to the BDM-H method. In particular, we have that the original BDM method is a BDM-H method; see [42].

3.3. The LDG-H methods. The LDG-H methods are obtained by using the LDG method to define the local solvers. The following specifications completely define the class of LDG-H methods:

1. The numerical traces

$$
\widehat{\mathbf{Q}} \mathrm{m}=\mathbf{Q} \mathrm{m}+\tau_{K}(U \mathrm{~m}-\mathrm{m}) \boldsymbol{n}, \quad \widehat{\mathbf{Q}} f=\mathbf{Q} f+\tau_{K}(U f) \boldsymbol{n} \quad \text { on } \partial K,
$$

where the $\tau_{K}$ is a function that can vary on $\partial K$.

2. The space $\boldsymbol{V}(K) \times W(K)$ as one of the following choices:

$$
\begin{aligned}
& \mathcal{P}_{k}(K)^{n} \times \mathcal{P}_{k-1}(K), k \geq 1 \text { and } \tau_{K} \geq 0 \text { on } \partial K, \\
& \mathcal{P}_{k}(K)^{n} \times \mathcal{P}_{k}(K), \quad k \geq 0 \text { and } \tau_{K}>0 \text { on at least one face of the simplex } K, \\
& \mathcal{P}_{k-1}(K)^{n} \times \mathcal{P}_{k}(K), k \geq 1 \text { and } \tau_{K}>0 \text { on } \partial K
\end{aligned}
$$

3. The space of approximate traces is

$$
\mathrm{M}_{h}=\mathcal{M}_{h, k} .
$$

Typically, the stabilization parameter $\tau$ of the LDG methods is a nonnegative constant on each face in $\mathcal{E}_{h}$. Here we allow $\tau$ to be double-valued on $\mathcal{E}_{h}^{\circ}$ with two branches $\tau^{-}=\tau_{K^{-}}$and $\tau^{+}=\tau_{K^{+}}$defined on the edge $e$ shared by the finite elements $K^{-}$and $K^{+}$. Now the functions $(\mathbf{Q m}, \mathfrak{U} \mathrm{m})$ and $(\mathbf{Q} f, \mathcal{U} f)$ are the approximations given by the LDG method to the exact solutions of (1.4) on each element, as claimed. As is well known, see $[34,17,5]$, the LDG method is uniquely solvable for $\tau_{K}>0$. However, the above specifications define a wider class of LDG-H methods. We show that the existence and uniqueness of the solution of the method can be guaranteed for each of the choices (3.5).

Proposition 3.2. The Assumption 2.1 on the existence and uniqueness of the local solvers holds for the numerical traces given by (3.4) and with any of the choices (3.5) for $\boldsymbol{V}(K) \times W(K)$.

To prove this result for all the above mentioned cases, we use the following auxiliary lemma.

Lemma 3.1. Let $\tau_{K} \geq 0$. With the choice of numerical traces in (3.4), the local problems (2.3) and (2.4) are uniquely solvable if $\boldsymbol{V}(K) \times W(K)$ defined by (3.5) is such that whenever $w \in W(K)$ satisfies

(i) $\tau_{K} w=0$ on $\partial K$, and

(ii) $(w, \operatorname{div} \boldsymbol{v})_{K}=0$ for all $\boldsymbol{v} \in \boldsymbol{V}(K)$, we have that $w=0$.

Proof. Let us prove the result for the first local solver $(\mathbf{Q m}, \mathcal{U m})$ defined by $(2.3)$. The result for the other local mapping (2.4) is similar. It suffices to prove uniqueness since this implies existence. To prove uniqueness, we must show that, when $m=0$, the only solution of (2.3) is the trivial one.

Taking $\boldsymbol{v}=\mathbf{Q m}$ and $w=\mathcal{U}_{\mathrm{m}}$ in (2.3), and adding the resulting equations, we get

$$
(c \mathbf{Q m}, \mathbf{Q m})_{K}+\langle U \mathrm{~m},(\widehat{\mathbf{Q}} \mathrm{m}-\mathbf{Q m}) \cdot \boldsymbol{n}\rangle_{\partial K}+\left(d \mathcal{U}, U_{\mathrm{m}}\right)_{K}=0 .
$$

Inserting the definition of the numerical trace $\widehat{\mathbf{Q}} \mathrm{m}$, we get

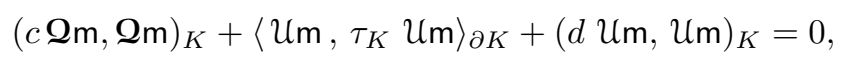


and since $c$ is positive definite and symmetric, $d \geq 0$ and $\tau_{K} \geq 0$, we have that $\mathbf{Q m}=\mathbf{0}$.

It remains to show that $U \mathrm{~m}=0$. To do so, we note that the above equation implies that $(\tau \mathcal{U m})_{K}=0$ on $\partial K$. By (2.3a), we also have

$$
(\mathcal{U m}, \operatorname{div} \boldsymbol{v})_{K}=0 \quad \text { for all } \boldsymbol{v} \in \boldsymbol{V}(K) .
$$

By hypothesis (ii), this implies that $\mathcal{U} \mathrm{m}=0$. This completes the proof. $\square$

We are now ready to prove Proposition 3.2.

Proof. By Lemma 3.1, we only have to show that, for each of the three choices (3.5), if $w \in W(K)$ satisfies $\tau_{K} w=0$ on $\partial K$ and $(w, \operatorname{div} \boldsymbol{v})_{K}=0$ for all $\boldsymbol{v}$ in $\boldsymbol{V}(K)$, then $w=0$ on $K$.

Let us show that this is true for the spaces given by (3.5a). Since div : $\boldsymbol{V}(K) \rightarrow W(K)$ is surjective, we know there is a $\boldsymbol{v}$ in $\boldsymbol{V}(K)$ such that $\operatorname{div} \boldsymbol{v}=w$. This implies that $(w, w)_{K}=0$, and hence that $w=0$ on $K$.

Next, let us consider the choice (3.5b). Since $w$ must vanish on the face $F$ where $\tau_{K}>0$, we immediately have that $w=0$ if $k=0$. If $k \geq 1$, it can be factored as $w=\ell_{F} p_{k-1}$, with $p_{k-1} \in \mathcal{P}_{k-1}(K)$ and $\ell_{F}$ equal to the barycentric coordinate function of $K$ that vanishes on $F$. Then, choosing $\boldsymbol{v}$ in $\boldsymbol{V}(K)=\mathcal{P}_{k}(K)^{n}$ such that $\operatorname{div} \boldsymbol{v}=p_{k-1}$, the equation

$$
0=(\operatorname{div} \boldsymbol{v}, w)_{K}=\left(\operatorname{div} \boldsymbol{v}, \ell_{F} p_{k-1}\right)_{K}=\left(p_{k-1}, \ell_{F} p_{k-1}\right)_{K}
$$

implies that $p_{k-1}$ vanishes on $K$, so $w=0$ on $K$.

Finally, let us consider the choice (3.5c). Since $\tau_{K}>0$ on $\partial K$, we have that $w=0$ on $\partial K$ and a simple integration by parts gives that

$$
(\operatorname{grad} w, \boldsymbol{v})_{K}=0 \quad \text { for all } \boldsymbol{v} \in \boldsymbol{V}(K)=\mathcal{P}_{k-1}(K)^{n} .
$$

Taking $\boldsymbol{v}=\operatorname{grad} w$ allows us to conclude that $w$ is a constant on $K$ and hence identically zero on $K$. This completes the proof. $\square$

Note that the choices (3.4) of the numerical traces, (3.5) of the finite elements spaces $\boldsymbol{V}(K) \times W(K)$ and (3.6) for the approximate trace space $\mathrm{M}_{h}$ clearly imply that, for all these LDG-H methods, the conservativity condition (2.6) is satisfied strongly. Moreover, by Theorem 2.1, the conservativity condition is equivalent to $a_{h}\left(\lambda_{h}, \mu\right)=b_{h}(\mu)$ for all $\mu \in M_{h}$, where

$$
\begin{aligned}
a_{h}(\eta, \mu) & =(c \mathbf{Q} \eta, \mathbf{Q} \mu)_{\mathcal{T}_{h}}+\left(d \mathcal{U} \eta, \mathcal{U}_{\mu}\right)_{\mathcal{T}_{h}}+\langle 1, \llbracket(\mathcal{U} \mu-\mu)(\tau(\mathcal{U} \eta-\eta) \boldsymbol{n}) \rrbracket\rangle_{\mathcal{E}_{h}} \\
b_{h}(\mu) & =\left\langle g_{h}, \mathbf{Q} \mu \cdot \boldsymbol{n}+\tau \mathcal{U} \mu\right\rangle_{\partial \Omega}+\left(f, \mathcal{U}_{\mu}\right)_{\mathcal{T}_{h}},
\end{aligned}
$$

provided $\left.g_{h}\right|_{\mathcal{E}_{h}^{\circ}}=0$.

The form $a_{h}(\cdot, \cdot)$ is obviously symmetric. That it is also positive definite follows once Assumption 2.2 on the positive semidefiniteness of the local solvers is verified. Set

$$
\begin{aligned}
M(\partial K)=\{\mu: \quad & \left.\mu\right|_{e} \in \mathcal{P}_{k}(e) \text { for all faces } e \text { where } \tau_{K}=0, \text { and } \\
& \left.\left.\mu\right|_{e} \in L^{2}(e) \text { for all faces } e \text { where } \tau_{K}>0\right\} .
\end{aligned}
$$

Proposition 3.3. Let the numerical traces be set by (3.4), the local spaces be as in any of the choices (3.5), and the space of approximate traces be set by (3.6). Then the Assumption 2.2 on the positive semidefiniteness of the local solvers and the Assumption 2.3, the gluing condition, are satisfied with $M(\partial K)$ defined by (3.7).

Proof. We begin by showing that the condition (2.13a) holds. By the identity (2.14) with $\mu:=\mathrm{m}$ and the definition of $\widehat{\mathbf{Q}} \mathrm{m}$, we have that

$$
-\langle\mathrm{m}, \widehat{\mathbf{Q}} \mathrm{m} \cdot \boldsymbol{n}\rangle_{\partial K}=(c \mathbf{Q m}, \mathbf{Q m})_{K}+\left(d \mathcal{U}, \mathcal{U m}_{K}+\left\langle\tau_{K}(\mathcal{U} \mathrm{m}-\mathrm{m}), \mathcal{U} \mathrm{m}-\mathrm{m}\right\rangle_{\partial K} .\right.
$$

Since $\tau_{K} \geq 0$ in all three cases (3.5), we see that the condition (2.13a) is satisfied.

Now, let us verify the condition (2.13b). If we assume that $\langle\mathrm{m}, \widehat{\mathbf{Q}} \mathrm{m} \cdot \boldsymbol{n}\rangle_{\partial K}=0$, we immediately obtain that $\left.\mathbf{Q m}\right|_{K}=0$ and $\left.\tau(\mathcal{U} \mathrm{m}-\mathrm{m})\right|_{\partial K}=0$. This implies that the first equation defining the first local solver, (2.3a), can be rewritten as

$$
(\operatorname{grad} U \mathrm{~m}, \boldsymbol{v})_{K}-\langle\mathcal{U} \mathrm{m}-\mathrm{m}, \boldsymbol{v} \cdot \boldsymbol{n}\rangle_{\partial K}=0 \quad \text { for all } \boldsymbol{v} \in \boldsymbol{V}(K)
$$


We use this equation to show that in all three cases (3.5) the condition $(2.13 \mathrm{~b})$ is satisfied with $P_{\partial K}$ defined, on the face $e$ of $K$, as the $L^{2}$-projection into $\mathcal{P}_{k}(e)$ if $\left.\tau\right|_{e}=0$ and as the identity if $\left.\tau\right|_{e}>0$ :

(i) In the case (3.5a), the result follows exactly as in the proof of Proposition 3.1.

(ii) In the case (3.5b), we know, see [24], that there is a function $\boldsymbol{v} \in \mathcal{P}_{k}(K)^{n}$ such that

$$
\begin{aligned}
\left(\boldsymbol{v}, \boldsymbol{p}_{k-1}\right)_{K} & =\left(\operatorname{grad} U \mathrm{~m}, \boldsymbol{p}_{k-1}\right)_{K}, & & \text { for all } \boldsymbol{p}_{k-1} \in \mathcal{P}_{k-1}(K)^{n} \\
\left\langle\boldsymbol{v} \cdot \boldsymbol{n}, p_{k}\right\rangle_{e} & =-\left\langle\mathcal{U}-\mathrm{m}, p_{k}\right\rangle_{e}, & & \text { for all } p_{k} \in \mathcal{P}_{k}(e),
\end{aligned}
$$

for all the faces $e$ of $K$ except one, say the face $e^{\prime}$ on which $\tau>0$. Setting this $\boldsymbol{v}$ in (3.8), and using the fact that on $e^{\prime}$ we have that $\mathrm{m}=\mathcal{U} \mathrm{m}$, we obtain that $U \mathrm{~m}$ is a constant on $K$, and that $\mathrm{m}=\mathcal{U} \mathrm{m}$ on the remaining faces of $\partial K$. Thus, $m$ is constant on $\partial K$ and condition (2.13b) is verified. Assumption 2.3, the gluing condition, is trivially satisfied by virtue of the definition of $M(\partial K)$ in (3.7).

(iii) In the case (3.5c), we immediately see that $\mathrm{m}=\mathcal{U} \mathrm{m}$ on $\partial K$. Now we take $\boldsymbol{v}=\operatorname{grad} U \mathrm{~m}$ in (3.8) to get that $\mathcal{U m}$ is a constant. This verifies Assumption 2.2 as in the previous case. Assumption 2.3 obviously holds from the definition of $M(\partial K)$ in (3.7).

Our next result sheds light into the nature of the numerical traces $\widehat{\boldsymbol{q}}_{h}$ and $\widehat{u}_{h}$ of the LDG-H schemes.

Proposition 3.4 (Characterization of LDG-H methods). Let the numerical traces be set by (3.4), the local spaces be as in any of the choices (3.5), the space of approximate traces be set by (3.6), and $\left(\boldsymbol{q}_{h}, u_{h}\right)$ be as defined in (2.5). Then the conservativity condition (2.6) holds on $\mathcal{E}_{h}^{\circ}$ if and only if

$$
\begin{aligned}
\lambda_{h}=\widehat{u}_{h} & =\left(\frac{\tau^{+}}{\tau^{-}+\tau^{+}}\right) u_{h}^{+}+\left(\frac{\tau^{-}}{\tau^{-}+\tau^{+}}\right) u_{h}^{-}+\left(\frac{1}{\tau^{+}+\tau^{-}}\right) \llbracket \boldsymbol{q}_{h} \rrbracket \\
\widehat{\boldsymbol{q}}_{h} & =\left(\frac{\tau^{-}}{\tau^{-}+\tau^{+}}\right) \boldsymbol{q}_{h}^{+}+\left(\frac{\tau^{+}}{\tau^{-}+\tau^{+}}\right) \boldsymbol{q}_{h}^{-}+\left(\frac{\tau^{+} \tau^{-}}{\tau^{-}+\tau^{+}}\right) \llbracket u_{h} \rrbracket .
\end{aligned}
$$

Proof. Suppose the conservativity condition holds. We need to prove (3.11a) and (3.11b). By the definition of $\widehat{\boldsymbol{q}}_{h}$, see (2.7), we have

$$
\begin{aligned}
\widehat{\boldsymbol{q}}_{h} & =\widehat{\mathbf{Q}} \lambda_{h}+\widehat{\mathbf{Q}} g_{h}+\widehat{\mathbf{Q}} f \\
& =\left(\mathbf{Q} \lambda_{h}+\mathbf{Q} g_{h}+\mathbf{Q} f\right)+\tau\left(\mathcal{U} \lambda_{h}+\mathcal{U} g_{h}+\mathcal{U} f-\lambda_{h}-g_{h}\right) \boldsymbol{n} \\
& =\boldsymbol{q}_{\boldsymbol{h}}+\tau\left(u_{h}-\lambda_{h}-g_{h}\right) \boldsymbol{n} .
\end{aligned}
$$

Inserting this expression into the conservativity condition, and taking $g_{h}$ equal to zero on $\mathcal{E}_{h}^{\circ}$, we obtain that for any $\mu \in M_{h}$,

$$
\left\langle\mu, \llbracket \widehat{\boldsymbol{q}}_{h} \rrbracket\right\rangle_{\varepsilon_{h}^{\circ}}=\left\langle\mu, \llbracket \boldsymbol{q}_{h}+\tau\left(u_{h}-\lambda_{h}\right) \boldsymbol{n} \rrbracket\right\rangle_{\varepsilon_{h}^{\circ}}=0,
$$

which implies, by our choice of spaces, that $\llbracket \widehat{\boldsymbol{q}}_{h} \rrbracket=0$ on $\mathcal{E}_{h}^{\circ}$, or, equivalently, that

$$
\llbracket \boldsymbol{q}_{h} \rrbracket+\left(\tau^{+} u_{h}^{+}+\tau^{-} u_{h}^{-}\right)-\left(\tau^{+}+\tau^{-}\right) \lambda_{h}=0 \text { on } \mathcal{E}_{h}^{\circ} .
$$

Solving for $\lambda_{h}$, we obtain (3.11a). To prove (3.11b), we simply insert the expression for $\lambda_{h}$ into the identity

$$
\widehat{\boldsymbol{q}}_{h}^{+} \cdot \boldsymbol{n}^{+}=\boldsymbol{q}_{h}^{+} \cdot \boldsymbol{n}^{+}+\tau^{+}\left(u_{h}^{+}-\lambda_{h}\right),
$$

and perform a few algebraic manipulations.

The converse asserted by the proposition is trivial: If the identities (3.11) hold, then the normal component of $\widehat{\boldsymbol{q}}_{h}$ is single valued on $\mathcal{E}_{h}^{\circ}$ and the conservativity condition is satisfied. This completes the proof.

Corollary 3.2. The $L D G$ method is not an $L D G-H$ method for any finite $\tau$.

Proof. On any interior face $e \in \mathcal{E}_{h}^{\circ}$, the LDG method has a numerical trace $\widehat{u}_{h}$ independent of $\boldsymbol{q}_{h}$, see [34, 17, 5]. On the other hand, by Proposition 3.4, the LDG-H methods have numerical traces $\widehat{u}_{h}$ that depend on $\llbracket \boldsymbol{q}_{h} \rrbracket$. Since this dependence cannot be removed for any finite value of $\tau$, we see that no LDG method is an LDG-H method. This completes the proof.

As known from [34, pp. 2445] and [17, pp. 1681], the independence of the numerical trace $\widehat{u}_{h}$ of the LDG methods of $\boldsymbol{q}_{h}$ on the interior faces $\mathcal{E}_{h}^{\circ}$ allows to eliminate the unknown $\boldsymbol{q}_{h}$ from the equations and to obtain a 
primal formulation involving only $u_{h}$. In contrast, in the LDG-H methods $\widehat{u}_{h}$ must depend on $\boldsymbol{q}_{h}$ as well. Both approaches recover $\boldsymbol{q}_{h}$ locally, but using different mechanisms. Since the LDG-H methods leads to a formulation involving only the numerical trace $\lambda_{h}$, they have fewer globally coupled unknowns than the LDG method for high order polynomials.

The LDG-H methods considered in this subsection were studied in [17] where it was proven, in particular, that the method is well defined for $\tau>0$ on $\varepsilon_{h}$. Methods with $\tau=0$ do not fit in the framework proposed in [5]; they have been recently studied in [24].

3.4. A limiting case of LDG-H methods. Here we consider hybridizable Galerkin methods that can be obtained formally considering limiting values of the penalty parameter in LDG-H methods. The motivation for doing this arises from the previous corollary (Corollary 3.2), whereby we know that the only chance for showing that an LDG method can be hybridized lies in cases where $\tau$ is allowed to be not finite.

We first examine how numerical traces of the previous LDG-H method change as we formally pass to a limit in $\tau$. By letting $\tau^{+}$go to infinity on the interior face $e=\partial K^{+} \cap \partial K^{-}$, while maintaining a fixed finite $\tau^{-}$, we find that the expressions for the numerical traces obtained in Proposition 3.4 become

$$
\widehat{u}_{h}=u_{h}^{+} \quad \text { and } \quad \widehat{\boldsymbol{q}}_{h}=\boldsymbol{q}_{h}^{-}+\tau^{-} \llbracket u_{h} \rrbracket .
$$

Note that the above expression for the primal numerical trace $\widehat{u}_{h}$ is independent of the fluxes, or in other words, such traces will result in an LDG method. Indeed, the LDG method defined by these numerical traces have been thoroughly studied in the case $\tau^{-}>0$, see $[34,17,5]$.

In the special case $\tau^{-}=0$, we get

$$
\widehat{u}_{h}=u_{h}^{+} \quad \text { and } \quad \widehat{\boldsymbol{q}}_{h}=\boldsymbol{q}_{h}^{-},
$$

which also defines a previously studied LDG method. For this scheme, the discontinuities of the approximate solution across interior inter-element boundaries do not introduce any dissipation. The dissipative effect of the discontinuities is concentrated on the boundary of the domain, and hence reduced to a "minimum", which is the reason for its name, the minimal dissipation LDG method. Since this scheme does not fit the unified analysis in [5], it was studied in [20] and [24] for problems in one and several space dimensions, respectively.

The formal passage to limit solely in the expressions for numerical traces does not clarify if the limiting methods are hybridizable. In particular, we must explain precisely what we mean by setting $\tau_{K}=\infty$ in the context of local solvers. To do so, let $F_{K}$ be the union of one or more faces of the element $K$ where we want to set the branch $\tau_{K}$ to $\infty$. Since

$$
\widehat{\mathbf{Q}} \mathrm{m}=\mathbf{Q} \mathrm{m}+\tau_{K}(\mathcal{U} \mathrm{m}-\mathrm{m}) \boldsymbol{n}
$$

we expect that in the formal limit of $\tau_{K}=\infty$, we should have $\mathcal{U}_{\mathrm{m}}-\mathrm{m}=0$. Then the value of $\widehat{\mathbf{Q}}_{\mathrm{m}}$ on $F_{K}$ becomes an unknown because the last term above is an unknown formal product of 0 with $\infty$. Motivated by this, we now define the local solvers with $\widehat{\mathbf{Q}} \mathrm{m}$ and $\widehat{\mathbf{Q}} f$ as new unknowns. More precisely, setting

$$
W(K)=\mathcal{P}_{k}(K), \quad \boldsymbol{V}(K)=\mathcal{P}_{k}(K)^{n}, \quad \boldsymbol{T}_{K}\left(F_{K}\right)=\left\{\left.\boldsymbol{n}_{K} w\right|_{F_{K}}: w \in W(K)\right\},
$$

we define the local solution $\left(\mathbf{Q} \mathrm{m}, \mathcal{U} \mathrm{m},(\widehat{\mathbf{Q}} \mathrm{m})_{F_{K}}\right) \in \boldsymbol{V}(K) \times W(K) \times \boldsymbol{T}_{K}\left(F_{K}\right)$ for any $\mathrm{m} \in \mathrm{M}_{h}$ by

$$
\begin{aligned}
& \quad(c \mathbf{Q m}, \boldsymbol{v})_{K}-(\mathcal{U} \mathrm{m}, \operatorname{div} \boldsymbol{v})_{K}=-\langle\mathrm{m}, \boldsymbol{v} \cdot \boldsymbol{n}\rangle_{\partial K} \quad \text { for all } \boldsymbol{v} \in \boldsymbol{V}(K), \\
& -(\operatorname{grad} w, \mathbf{Q m})_{K}+\langle w, \widehat{\mathbf{Q}} \mathrm{m} \cdot \boldsymbol{n}\rangle_{\partial K}+(d \mathcal{U}, w)_{K}=0 \text { for all } w \in W(K), \\
& \quad \mathcal{m}=\mathrm{m} \quad \text { on } F_{K} .
\end{aligned}
$$

Here, just as for the LDG-H methods, we set

$$
\widehat{\mathbf{Q}} \mathrm{m}=\mathbf{Q} \mathrm{m}+\tau_{K}(\mathcal{U} \mathrm{m}-\mathrm{m}) \boldsymbol{n}, \quad \text { on } \partial K \backslash F_{K} .
$$

Similarly, we define $\left(\mathbf{Q} f, \mathcal{U} f,(\widehat{\mathbf{Q}} f)_{F_{K}}\right)$ as the element of $\boldsymbol{V}(K) \times W(K) \times \boldsymbol{T}_{K}\left(F_{K}\right)$ such that

$$
\begin{array}{cr}
(c \mathbf{Q} f, \boldsymbol{v})_{K}-(\mathcal{U} f, \operatorname{div} \boldsymbol{v})_{K}=0 & \text { for all } \boldsymbol{v} \in \boldsymbol{V}(K), \\
-(\operatorname{grad} w, \boldsymbol{Q} f)_{K}+\langle w, \widehat{\mathbf{Q}} f \cdot \boldsymbol{n}\rangle_{\partial K}+(d \mathcal{U} f, w)_{K}=(f, w) & \text { for all } w \in W(K), \\
& \mathcal{U} f=0 \quad \text { on } F_{K},
\end{array}
$$


where

$$
\widehat{\mathbf{Q}} f=\mathbf{Q} f+\tau_{K}(\mathcal{U} f) \boldsymbol{n} \quad \text { on } \partial K \backslash F_{K} .
$$

We set the space of approximate traces by

$$
\mathrm{M}_{h}=\left\{\mu \in \mathcal{M}_{h, k}:\left.\mu\right|_{F_{K}} \text { is continuous on } F_{K} \text { for all } K \in \mathcal{T}_{h}\right\} .
$$

Note that the continuity condition in the above definition reflects the fact that the local solvers satisfy a strong Dirichlet boundary conditions on $F_{K}$ for all $K \in \mathcal{T}_{h}$; see (3.13c) and (3.14c). This completes the definition of the limiting case of LDG-H method when $\tau_{K}=\infty$ on $F_{K}$. From now on, the above modification of the LDG local solvers is tacitly understood whenever we say that a branch of $\tau$ is infinity on a face. It is easy to check by arguments similar to that in Proposition 3.2, that the local problems (3.13) and (3.14) are uniquely solvable for every $\mathrm{m}$ in $\mathrm{M}_{h}$ and every $f \in L^{2}(\Omega)$ provided, for each element $K \in \mathcal{T}_{h}, \tau_{K}$ is not identically equal to zero on $\partial K$ whenever $F_{K}$ is the empty set.

Note that, although the local solvers have been modified, Theorem 2.1 continues to apply because its proof only relies on the form of the first two equations in the local problems. Indeed, the equations (3.13a) and (3.13b) are identical in form to the equations $(2.3 \mathrm{a})$ and $(2.3 \mathrm{~b})$, respectively; a similar remark applies to the equation of the second local solvers. Therefore Theorem 2.1 also holds in this case. In particular, we have that

$$
a_{h}(\eta, \mu)=(c \mathbf{Q} \eta, \mathbf{Q} \mu)_{\mathcal{T}_{h}}+\left(d \mathcal{U} \eta, \mathcal{U}_{\mu}\right)_{\mathcal{T}_{h}}+\sum_{K \in \mathcal{T}_{h}}\langle\tau(\mathcal{U} \eta-\eta),(\mathcal{U} \mu-\mu)\rangle_{\partial K \backslash F_{K}} .
$$

Finally, it is not difficult to see that Proposition 3.3 also holds. By Theorem 2.4, the bilinear form $a_{h}(\cdot, \cdot)$ is positive definite and we can immediately see that $\lambda_{h}$ is uniquely determined.

Note that, unlike all previous examples, the conservativity condition (2.6) for these methods is only imposed weakly. This is because while the jumps of $\widehat{\boldsymbol{q}}_{h}$ lie in $\mathcal{M}_{h, k}$, the approximate traces $\mu$ are in the space $\mathrm{M}_{h}$ which is a strict subspace of $\mathcal{M}_{h, k}$. Since all LDG methods have single-valued numerical traces, this seems to suggest that no LDG method can be a limiting case of the LDG-H method. However, this is not the case, as we see next.

We consider the one-sided limiting case of the $L D G-H$ method. This is the same as the above defined limiting case of LDG-H method, but with the following additional assumption: For every interior face $e$ in $\mathcal{E}_{h}^{\circ}$, one branch of $\tau$ is infinity, and the other branch is finite valued.

COROLLARY 3.3. The one-sided limiting case of the LDG-H method coincides with the LDG method whose numerical traces on the interior faces are given by (3.12).

Proof. Let $\lambda_{h}^{\infty}$ denote the solution of the one-sided limiting case of the LDG-H method and let

$$
\boldsymbol{q}_{h}^{\infty}=\mathbf{Q} \lambda_{h}^{\infty}+\mathbf{Q} g_{h}+\mathbf{Q} f, \quad u_{h}^{\infty}=\mathfrak{U} \lambda_{h}^{\infty}+\mathcal{U} g_{h}+\mathcal{U} f .
$$

We will prove that $\boldsymbol{q}_{h}^{\infty}$ and $u_{h}^{\infty}$ coincides with the corresponding solution components $\boldsymbol{q}_{h}^{\mathrm{LDG}}$ and $u_{h}^{\mathrm{LDG}}$, respectively, of the LDG method with numerical traces set as in (3.12).

By the definition of the LDG method, $\boldsymbol{q}_{h}^{\text {LDG }}$ and $u^{\text {LDG }}$ satisfy $(2.8 \mathrm{a})-(2.8 \mathrm{~b})$ with the $\lambda_{h}$ and $\widehat{\boldsymbol{q}}_{h}$ therein set, respectively, to $\widehat{u}_{h}$ and $\widehat{\boldsymbol{q}}_{h}$ of (3.12), which for clarity, we will rewrite as $\widehat{u}_{h}^{\mathrm{LDG}}$ and $\widehat{\boldsymbol{q}}_{h}^{\mathrm{LDG}}$.

It suffices to show that $\boldsymbol{q}_{h}^{\infty}$ and $u_{h}^{\infty}$ satisfy the same equations as $\boldsymbol{q}_{h}^{\text {LDG }}$ and $u_{h}^{\text {LDG }}$. Adding the local solver equations (3.13a) and (3.14a) over all elements, we find that $\boldsymbol{q}_{h}^{\infty}$ and $u_{h}^{\infty}$ satisfy the first equation of the LDG method with $\lambda_{h}^{\infty}$ in place of $\widehat{u}_{h}^{\mathrm{LDG}}$. But since every interior edge has an infinite penalty branch, and since

$$
\left.\lambda_{h}^{\infty}\right|_{F_{K}}=\left(u_{h}^{\infty}\right)_{F_{K}}, \quad \text { for all elements } K,
$$

we find that $\lambda_{h}^{\infty}$ is in the same form as the LDG numerical trace $\widehat{u}_{h}^{\mathrm{LDG}}$.

Also summing the local solver equations (3.13b) and (3.14b) over all elements, we find that $\boldsymbol{q}_{h}^{\infty}$ and $u_{h}^{\infty}$ satisfy the second equation of the LDG methods with $\widehat{\boldsymbol{q}}_{h}^{\infty} \equiv \widehat{\mathbf{Q}} \lambda_{h}^{\infty}+\widehat{\mathbf{Q}} g_{h}+\widehat{\mathbf{Q}} f$ in place of $\widehat{\boldsymbol{q}}_{h}^{\text {LDG }}$. We will now show that the second equation in fact holds with the LDG flux. For this, we use the fact that

$$
\left\langle\llbracket \widehat{\boldsymbol{q}}_{h}^{\infty} \rrbracket, \mu\right\rangle_{\varepsilon_{h}}=0,
$$


for all $\mu$ in the subspace $M_{h}$ of functions in $\mathrm{M}_{h}$ (defined by (3.15)) with $\left.\mu\right|_{\partial \Omega}=0$. Now, if $w$ is any function in $W(K)$, then $\left.w\right|_{F_{K}}$, extended by zero to $\mathcal{E}_{h}$, is in $M_{h}$. Therefore (3.17) implies

$$
\begin{aligned}
\left\langle\widehat{\boldsymbol{q}}_{h}^{\infty} \cdot \boldsymbol{n}, w\right\rangle_{F_{K}} & =-\left\langle\left(\widehat{\boldsymbol{q}}_{h}^{\infty}\right)_{K^{c}} \cdot(\boldsymbol{n})_{K^{c}}, w\right\rangle_{F_{K}} \\
& =-\left\langle\left(\widehat{\boldsymbol{q}}_{h}^{\infty}\right)_{K^{c}}+(\tau)_{K^{c}}\left(\left(u_{h}^{\infty}\right)_{K^{c}}-\lambda_{h}^{\infty}\right)(\boldsymbol{n})_{K^{c}},(\boldsymbol{n})_{K^{c}} w\right\rangle_{F_{K}} .
\end{aligned}
$$

Here, for notational convenience, we have denoted the branch of a multivalued function $f$ from outside $K$ by $(f)_{\mathrm{K}^{c}}$. By (3.16), we can rewrite the right hand side as

$$
\left\langle\widehat{\boldsymbol{q}}_{h}^{\infty} \cdot \boldsymbol{n}, w\right\rangle_{F_{K}}=-\left\langle\left(\widehat{\boldsymbol{q}}_{h}^{\infty}\right)_{K^{c}}+(\tau)_{K^{c}} \llbracket u_{h}^{\infty} \rrbracket,(\boldsymbol{n})_{K^{c}} w\right\rangle_{F_{K}},
$$

and conclude that

$$
\sum_{K}\left\langle\widehat{\boldsymbol{q}}_{h}^{\infty} \cdot \boldsymbol{n}, w\right\rangle_{\partial K}=\sum_{K}\left\langle\widehat{\boldsymbol{q}}_{h}^{\mathrm{LDG}} \cdot \boldsymbol{n}, w\right\rangle_{\partial K} \cdot
$$

Thus $\boldsymbol{q}_{h}^{\infty}$ and $u_{h}^{\infty}$ satisfy the same equations as the LDG method with the same expressions for numerical traces as in the LDG case.

Note that in the above proof $\widehat{\boldsymbol{q}}_{h}^{\infty}$ and $\widehat{\boldsymbol{q}}_{h}^{\text {LDG }}$ are not identical, in general, although (3.18) holds. This explains why the normal component of the limiting LDG-H numerical trace may not be single valued, although the numerical trace of its equivalent LDG method is single valued.

3.5. The CG-H method. The CG-H methods are obtained by using the CG method to define the local solvers. We are also going to see that they are also obtained from LDG-H methods by letting $\tau$ go to infinity everywhere.

Again, we need to specify the main ingredients of the local solvers. Similarly to the the limiting case of LDG-H methods we need to give a new meaning of the local solvers since $\tau=\infty$. Since the numerical flux $\widehat{\mathbf{Q}}$. will be unknown we need an appropriate space for its approximation.

1. For any $k \geq 1$, and any $K \in \mathcal{T}_{h}$, we define the finite element spaces by

$$
\boldsymbol{V}(K)=\mathcal{P}_{k-1}(K)^{n}, \quad W(K)=\mathcal{P}_{k}(K), \quad \text { and } \boldsymbol{T}(\partial K):=\left\{\left.\boldsymbol{n}_{K} w\right|_{\partial K}: w \in W(K)\right\} ;
$$

2. the numerical traces of the fluxes $\widehat{\mathbf{Q}}$. are unknown and will be determined by the modified local solvers as follows: $(\mathbf{Q m}, \mathcal{U}, \widehat{\mathbf{Q}} \mathrm{m}) \in \boldsymbol{V}(K) \times W(K) \times \boldsymbol{T}(\partial K)$ is a solution to the problem

$$
\begin{array}{lr}
(c \mathbf{Q m}, \boldsymbol{v})_{K}-(\mathcal{U} \mathrm{m}, \operatorname{div} \boldsymbol{v})_{K}=-\langle\mathrm{m}, \boldsymbol{v} \cdot \boldsymbol{n}\rangle_{\partial K}, & \forall \boldsymbol{v} \in \boldsymbol{V}(K), \\
-(\operatorname{grad} w, \mathbf{Q m})_{K}+\langle w, \widehat{\mathbf{Q}} \mathrm{m} \cdot \boldsymbol{n}\rangle_{\partial K}+(d \mathcal{U}, w)_{K}=0, & \forall w \in W(K), \\
\boldsymbol{U} \mathrm{m}=\mathrm{m}, \quad \text { on } \partial K, &
\end{array}
$$

Similarly, $(\mathbf{Q} f, \mathcal{u} f, \widehat{\mathbf{Q}} f) \in \boldsymbol{V}(K) \times W(K) \times \boldsymbol{T}(\partial K)$ is defined by

$$
\begin{aligned}
& (c \mathbf{Q} f, \boldsymbol{v})_{K}-(\mathcal{U} f, \operatorname{div} \boldsymbol{v})_{K}=0, \quad \forall \boldsymbol{v} \in \boldsymbol{V}(K), \\
- & (\operatorname{grad} w, \mathbf{Q} f)_{K}+\langle w, \widehat{\mathbf{Q}} f \cdot \boldsymbol{n}\rangle_{\partial K}+(d \mathcal{U} f, w)_{K}=(f, w)_{K}, \quad \forall w \in W(K), \\
& \mathcal{U} f=0, \quad \text { on } \partial K ;
\end{aligned}
$$

3. for the space of approximate traces, we take

$$
\mathrm{M}_{h}:=\mathcal{M}_{h, k}^{c} .
$$

We begin our discussion regarding the above CG-H method by verifying the assumptions required by Theorem 2.4.

Proposition 3.5. Assumption 2.1 on the existence and uniqueness of the local solvers holds for the CG-H local solver. Assumption 2.2 on the positive semidefiniteness of the local solvers and Assumption 2.3, the gluing condition, hold with $M(\partial K)=L^{2}(\partial K)$.

Proof. We prove the result for the local solver $(\mathbf{Q m}, \mathcal{U}, \widehat{\mathbf{Q}} \mathrm{m})$ defined by (3.20). The result for the local mapping defined by (3.21) is similar. Since the resulting system is square, we only prove uniqueness since this implies existence. Thus, we need to show that if $\mathrm{m}=0$ then the only solution is the trivial one. 
Taking $\boldsymbol{v}=\mathbf{Q m}$ in (3.20a) and $w=\mathcal{U} \mathrm{m}$ in (3.20b) and adding the resulting equations, we get

$$
(c \mathbf{Q m}, \mathbf{Q} \mathrm{m})_{K}+\langle\mathcal{U} \mathrm{m},(\widehat{\mathbf{Q}} \mathrm{m}-\mathbf{Q} \mathrm{m}) \cdot \boldsymbol{n}\rangle_{\partial K}+\left(d \mathcal{U}, \mathcal{U m}_{K}=0\right.
$$

Since, by the equation $(3.20 \mathrm{c}), \mathcal{U} \mathrm{m}=0$ on $\partial K$, we immediately obtain that $\mathbf{Q m}=\mathbf{0}$. This implies that the equation (3.20a) can be rewritten as follows:

$$
(\operatorname{grad} U \mathrm{~m}, \boldsymbol{v})_{K}=0 \quad \text { for all } \boldsymbol{v} \in \boldsymbol{V}(K)
$$

which implies that $\mathcal{U} \mathrm{m}=0$.

It remains to show that $\widehat{\mathbf{Q}} \mathrm{m}=0$. To do that, we use the equation $(3.20 \mathrm{~b})$ rewritten as

$$
\langle w, \widehat{\mathbf{Q}} \mathbf{m} \cdot \boldsymbol{n}\rangle_{\partial K}=0 \quad \text { for all } w \in W(K) .
$$

By the definition of the space $\boldsymbol{T}(\partial K)$, we can find a function $w \in W(K)$ such that $\widehat{\mathbf{Q}} \mathrm{m}=w \boldsymbol{n}$. This readily implies that $\widehat{\mathbf{Q}} \mathrm{m}=0$. This completes the verification of Assumption 2.1.

Inequality (2.13a) of Assumption 2.2 can easily be seen to hold. The second part of Assumption 2.2 also holds since $M(\partial K)=L^{2}(\partial K)$. Finally, Assumption 2.3 trivially holds. $\square$

Next, we discuss the conservativity condition. The flux approximation $\boldsymbol{q}_{h}$ of the CG-H method is, in general, not in $H(\operatorname{div}, \Omega)$. Nonetheless, it is interesting to observe that even the CG-H method has a weak conservativity property. This property holds for the numerical flux trace $\widehat{\boldsymbol{q}}_{h}=\widehat{\mathbf{Q}} \lambda_{h}+\widehat{\mathbf{Q}} g_{h}+\widehat{\mathbf{Q}} f$, a quantity that is not present in the standard formulations of the CG methods, but essential in our approach. Indeed, Theorem 2.1 asserts that $\widehat{\boldsymbol{q}}_{h}$ satisfies

$$
\left\langle\mu, \llbracket \widehat{\boldsymbol{q}}_{h} \rrbracket\right\rangle_{\varepsilon_{h}^{\circ}}=0, \quad \text { for all } \mu \in \mathrm{M}_{h}
$$

which is a weak conservativity condition.

Observe that if $a$ is a constant matrix on each element, by the definition of the local solvers (3.20) and (3.21), we have that

$$
\mathcal{Q} \mathrm{m}=-a \operatorname{grad} U \mathrm{~m} \quad \text { and } \quad \mathcal{Q} f=-a \operatorname{grad} \mathcal{U} f
$$

Hence $\boldsymbol{q}_{h}$ in $(2.8 \mathrm{a})$, being the sum of the local flux solutions, equals $-a$ grad $u_{h}$ on each element. Substituting this in (2.8b) and using the conservativity condition, we immediately see that $u_{h}$ satisfies the standard CG equations. In addition, by the boundary conditions defining the local solvers (3.20c) and (3.21c) imply that $u_{h}$ is continuous. Thus, we conclude that this CG-H formulation coincides with the CG method whenever $a$ is constant. In other words, the original CG method is a CG-H method when the matrix-valued function $a$ is a constant on each element. In this case we can also simplify the forms in (2.9) using (3.23) to

$$
\begin{aligned}
a_{h}(\eta, \mu) & =\left(a \operatorname{grad} U \eta, \operatorname{grad} U(\mu)_{\mathcal{T}_{h}}+(d \mathcal{U} \eta, \mathcal{U} \mu)_{\mathcal{T}_{h}},\right. \\
b_{h}(\mu) & =\left\langle g_{h}, \llbracket \widehat{\mathbf{Q}} \mu \rrbracket\right\rangle_{\mathcal{E}_{h}}+(f, \mathcal{U})_{\mathcal{T}_{h}} .
\end{aligned}
$$

Note that in our case we do not necessarily have that $\left.g_{h}\right|_{\mathcal{E}_{h}^{\circ}}=0$. Hence, the corresponding integral cannot be performed only on $\partial \Omega$ as in the previous cases.

The formulation (2.9) is nothing but the weak formulation for the continuous Galerkin method with static condensation of its interior degrees of freedom. This hybridization approach for the CG methods of degree $k$ is explored in [31], where, in particular, a postprocessing technique providing a locally conservative flux approximations competitive with that given by the RT methods of degree $k-1$ is introduced.

When the matrix-valued funcion $a$ is not constant on each element, we cannot write (3.23) anymore. Instead, " $a$ " has to be replaced by a function "a" which is, roughly speaking, the inverse of some local average of $c$, the inverse of $a$. In practice, however, we do not compute the matrix-valued function a; instead, we compute directly the functions $\mathbf{Q m}$ and $\mathbf{Q} f$ by using the definition of the local solvers. 
3.6. IP-H methods. The IP-H methods are obtained by using the numerical traces and the local solvers of the IP method. Thus,

1. the numerical traces are given by

$$
\widehat{\mathbf{Q}} \mathrm{m}=-\operatorname{agrad} U \mathrm{~m}+\tau_{K}(U \mathrm{~m}-\mathrm{m}) \boldsymbol{n}, \quad \widehat{\mathbf{Q}} f=-\operatorname{agrad} U f+\tau_{K}(U f) \boldsymbol{n}, \quad \text { on } \partial K ;
$$

2. the finite element space $\boldsymbol{V}(K) \times W(K)$ is defined for $k \geq 1$ as

$$
\boldsymbol{V}(K)=\mathcal{P}_{k}(K)^{n}, \quad W(K)=\mathcal{P}_{k}(K)
$$

3. the space of approximate traces is chosen as

$$
\mathrm{M}_{h}:=\mathcal{M}_{h, k}
$$

As before, $\tau$ is a double-valued on $\mathcal{E}_{h}^{\circ}$ with two branches $\tau^{-}=\tau_{K^{-}}$and $\tau^{+}=\tau_{K^{+}}$defined on the edge $e$ shared by the finite elements $K^{-}$and $K^{+}$.

Note that IP methods can be defined by using a flux formulation, as the one employed here to define the local solvers, or by means of a primal formulation; see [5]. These two IP methods, however, do coincide whenever the function $a$ is a constant on each element $K \in \mathcal{T}_{h}$. For this reason, we are going to assume here that this is the case. All the results for this case, however, can be easily extended to the case in which $a$ is not necessarily piecewise constant.

Next, we provide sufficient conditions for the IP-H method to be well defined. For simplicity, we assume that the mesh $\mathcal{T}_{h}$ is shape regular, that is, that there is a constant $\gamma>0$ such that $h_{K} / \rho_{K} \leq \gamma$ for all simplexes $K \in \mathcal{T}_{h}$, where $h_{K}$ is the diameter of $K$ and $\rho_{K}$ the diameter of the largest ball contained in $K$.

Proposition 3.6. Let the numerical traces be given by (3.24) and the local spaces by (3.25). Suppose a (x) is a constant matrix on each element $K$. Then Assumption (2.1) on the existence and uniqueness of the local solvers holds provided $\tau_{K}>c_{0} / h_{K}$ for some constant $c_{0}>0$ depending on $\gamma$ and $a(\boldsymbol{x})$.

For a proof, see $[6,3]$. Having established that the local solvers are well defined, we can apply Theorem 2.1. We find that the conservativity condition implies that $\lambda_{h}$ solves $(2.9)$ with

$$
\begin{aligned}
& a_{h}(\eta, \mu)=\left(c \mathbf{Q} \eta, \mathbf{Q}_{\mu}\right)_{\mathcal{T}_{h}}+(d \mathcal{U} \eta, \mathcal{U} \mu)_{\mathcal{T}_{h}} \\
&+\langle 1, \llbracket(\mu-\mathcal{U} \mu)(a \operatorname{grad} \mathcal{U} \eta+\mathbf{Q} \eta) \rrbracket\rangle_{\mathcal{E}_{h}} \\
&+\langle 1, \llbracket(\mathcal{U} \mu-\mu)(\tau(\mathcal{U} \eta-\eta) \boldsymbol{n}) \rrbracket\rangle_{\mathcal{E}_{h}} . \\
& b_{h}(\mu)=\left\langle g_{h},-\operatorname{agrad} \mathcal{U} \mu \cdot \boldsymbol{n}+\tau \mathcal{U} \mu\right\rangle_{\partial \Omega}+(f, \mathcal{U} \mu)_{\mathcal{T}_{h}},
\end{aligned}
$$

provided $\left.g_{h}\right|_{\mathcal{E}_{h}^{\circ}}=0$. Using $(2.12 \mathrm{a})$ of Lemma 2.3 and the fact that $a(\boldsymbol{x})$ is constant on each $K$, we can simplify this expression as follows:

$$
\begin{aligned}
a_{h}(\eta, \mu)= & \left(c \mathbf{Q} \eta, \mathbf{Q}_{\mu}\right)_{\mathcal{T}_{h}}-\left(c \mathbf{Q} \eta+\operatorname{grad} \mathcal{U} \eta, \mathbf{Q}_{\mu}+\operatorname{agrad} \mathcal{U}_{\mu}\right)_{\mathcal{T}_{h}}+\left(d \mathcal{U} \eta, \mathcal{U}_{\mu}\right)_{\mathcal{T}_{h}} \\
& +\langle 1, \llbracket(\mathcal{U} \mu-\mu)(\tau(\mathcal{U} \eta-\eta) \boldsymbol{n}) \rrbracket\rangle_{\mathcal{E}_{h}} \\
= & \left(a \operatorname{grad} \mathcal{U} \eta, \operatorname{grad} \mathcal{U}_{\mu}\right)_{\mathcal{T}_{h}}+(d \mathcal{U} \eta, \mathcal{U} \mu)_{\mathcal{T}_{h}} \\
& +\left\langle 1, \llbracket\left((\mathcal{U} \eta-\eta) a \operatorname{grad} \mathcal{U} \mu+(\mathcal{U} \mu-\mu) a \operatorname{grad} \mathcal{U}_{\eta} \eta\right) \rrbracket\right\rangle_{\mathcal{E}_{h}} \\
& +\langle 1, \llbracket(\mathcal{U} \mu-\mu)(\tau(\mathcal{U} \eta-\eta) \boldsymbol{n}) \rrbracket\rangle_{\mathcal{E}_{h}}
\end{aligned}
$$

The positive definiteness of the form $a_{h}(\cdot, \cdot)$ can be proven as in the case of LDG-H methods. Indeed, this fact is an immediate consequence of Theorem 2.4 and the following result.

Proposition 3.7. Let the numerical traces of the fluxes be set by (3.24), the local spaces be defined by (3.25), and the space of approximate traces be set by (3.26). Suppose a $(\boldsymbol{x})$ is a constant matrix on each element $K$. Then Assumption 2.2 on the positive semidefiniteness of the local solvers and Assumption 2.3, the gluing condition, are satisfied with $M(\partial K)=\left\{\mu:\left.\mu\right|_{e} \in \mathcal{P}_{k}(e)\right.$ for all faces $\left.e \in \partial K\right\}$ whenever $\tau_{K}>c_{0} / h_{K}$ for some constant $c_{0}>0$ depending on $\gamma$ and $a(\boldsymbol{x})$.

The proof of this result is similar to that of Proposition 3.3.

Just as for LDG-H methods we can give a characterization of the IP-H methods. It is given in the proposition below which is an analog of Proposition 3.4 for the LDG-H methods. Since the proof is similar, we omit it. 
Proposition 3.8 (Characterization of IP-H methods). Let the numerical traces be set by (3.24), the spaces be as in (3.25), and $\left(\boldsymbol{q}_{h}, u_{h}\right)$ be as defined in (2.5). Then the conservativity condition (2.6) holds if and only if on $\mathcal{E}_{h}^{\circ}$,

$$
\begin{aligned}
\lambda_{h}=\widehat{u}_{h} & =\left(\frac{\tau^{+}}{\tau^{-}+\tau^{+}}\right) u_{h}^{+}+\left(\frac{\tau^{-}}{\tau^{-}+\tau^{+}}\right) u_{h}^{-}-\left(\frac{1}{\tau^{+}+\tau^{-}}\right) \llbracket a \operatorname{grad} u_{h} \rrbracket \\
\widehat{\boldsymbol{q}}_{h} & =-\left(\frac{\tau^{-}}{\tau^{-}+\tau^{+}}\right) a^{+} \operatorname{grad} u_{h}^{+}-\left(\frac{\tau^{+}}{\tau^{-}+\tau^{+}}\right) a^{-} \operatorname{grad} u_{h}^{-}+\left(\frac{\tau^{+} \tau^{-}}{\tau^{-}+\tau^{+}}\right) \llbracket u_{h} \rrbracket .
\end{aligned}
$$

We also have a results analogous to Corollary 3.2 .

Corollary 3.4. The standard IP method is not an IP-H method for any finite $\tau$.

Proof. Comparing the numerical traces of the standard IP method, see [5, Table 3.1], namely,

$$
\widehat{u}_{h}^{\mathrm{IP}}=\left\{\left\{u_{h}\right\}\right\} \text { and } \widehat{\boldsymbol{q}}_{h}^{\mathrm{IP}}=-\left\{\left\{a \operatorname{grad} u_{h}\right\}\right\}+C \llbracket u_{h} \rrbracket,
$$

with the expressions for the numerical traces in Proposition 3.8, we find that they cannot coincide for any value of $\tau$. $\square$

In spite of this negative result, a stabilized DG finite element method introduced in [38], and rewritten in [37] as an IP method, turns out to be an IP-H method. To describe this scheme in a simple setting, assume that $d=0$ and $g=0$. The method, as presented in [38], does not use the function $\lambda_{h}$ approximating $\left.u\right|_{\mathcal{E}_{h}}$. Instead, it uses approximate fluxes $\ell_{h}$ approximating the normal component of $a \operatorname{grad} u$. The space in which $\ell_{h}$ lies is the space of scalar double-valued functions defined by

$$
L_{h}=\left\{q:\left.\quad q\right|_{e} \in \mathcal{P}_{k}(e) \text { for all } e \in \mathcal{E}_{h}, \text { and } q_{K^{+}}+q_{K^{-}}=0 \text { on } e=\partial K^{+} \cap \partial K^{-}\right\} .
$$

The DG method of [38] seeks $u_{h} \in W_{h}$, given by $(2.1)$ with $\left.W(K)=\mathcal{P}_{k}(K)\right)$, and $\ell_{h} \in L_{h}$ such that

$$
\begin{aligned}
\sum_{K \in \mathcal{T}_{h}}\left\{\left(a \operatorname{grad} u_{h}, \operatorname{grad} v\right)_{K}-\left\langle\ell_{h}, v\right\rangle_{\partial K}-\left\langle\eta, u_{h}\right\rangle_{\partial K}\right\} \\
-\alpha h \sum_{K \in \mathcal{T}_{h}}\left\langle\ell_{h}-a \operatorname{grad} u_{h} \cdot \boldsymbol{n}_{K}, \eta-a \operatorname{grad} v \cdot \boldsymbol{n}_{K}\right\rangle_{\partial K}=(f, v)
\end{aligned}
$$

for all $v \in W_{h}$ and $\eta \in L_{h}$. Here $\alpha>0$ is a constant stabilization parameter and $h=\max _{K \in \mathcal{T}_{h}} h_{K}$.

Taking $v \equiv 0$ and using that $\{\{\eta\}\}=0$ on $\mathcal{E}_{h}^{\circ}$ we get

$$
\ell_{h}= \begin{cases}\left\{\left\{a \operatorname{grad} u_{h}\right\}\right\} \cdot \boldsymbol{n}-\frac{1}{2 \alpha h} \llbracket u_{h} \rrbracket \cdot \boldsymbol{n}, & \text { on } \mathcal{E}_{h}^{\circ}, \\ a \operatorname{grad} u_{h} \cdot \boldsymbol{n}-\frac{1}{\alpha h} u_{h}, & \text { on } \mathcal{E}_{h}^{\partial} .\end{cases}
$$

We see from the above equation that $\ell_{h}$ is indeed an approximation to the normal component of $a$ grad $u$. Next, taking $\eta \equiv 0$ in (3.28) and substituting therein the expression for $\ell_{h}$ from (3.29), we get that $u_{h} \in W_{h}$ satisfies

$$
\begin{aligned}
\left(a \operatorname{grad} u_{h}, \operatorname{grad} v\right)_{\mathcal{T}_{h}} & -\left\langle\left\{\{a \operatorname{grad} v\}, \llbracket u_{h} \rrbracket\right\rangle_{\varepsilon_{h}}\right. \\
& -\left\langle\left\{\left\{a \operatorname{grad} u_{h}\right\}\right\}-\frac{1}{2 \alpha h} \llbracket u_{h} \rrbracket, \llbracket v \rrbracket\right\rangle_{\mathcal{E}_{h}^{\circ}} \\
& -\left\langle a \operatorname{grad} u_{h}-\frac{1}{\alpha h} u_{h} \boldsymbol{n}, v \boldsymbol{n}\right\rangle_{\varepsilon_{h}^{\partial}} \\
& -\left\langle\frac{\alpha h}{2} \llbracket a \operatorname{grad} u_{h} \rrbracket, \llbracket a \operatorname{grad} v \rrbracket\right\rangle_{\mathcal{E}_{h}^{\circ}}=(f, v),
\end{aligned}
$$

for all $v \in W_{h}$.

Now, we show that this is an IP-H method. Comparing the above formulation with the general primal formulation given by [5, equation (3.11)], we can easily verify that if we take

$$
\begin{array}{ll}
\widehat{u}_{h}=\left\{\left\{u_{h}\right\}-\frac{\alpha h}{2} \llbracket a \operatorname{grad} u_{h} \rrbracket\right. & \text { on } \mathcal{E}_{h}^{\circ}, \\
\widehat{\boldsymbol{q}}_{h}= \begin{cases}-\left\{\left\{a \operatorname{grad} u_{h}\right\}\right\}+\frac{1}{2 \alpha h} \llbracket u_{h} \rrbracket & \text { on } \mathcal{E}_{h}^{\circ}, \\
-a \operatorname{grad} u_{h}+\frac{1}{\alpha h} u_{h} \boldsymbol{n} & \text { on } \mathcal{E}_{h}^{\partial},\end{cases}
\end{array}
$$


we recover (3.30). Hence the above numerical traces are exactly the numerical traces of the IP-H method given by Proposition 3.8 with $\tau^{+}=\tau^{-}=(\alpha h)^{-1}$. This shows that the DG method proposed in [38] is an IP-H method. The correspondence between their flux approximation $\ell_{h}$ and our numerical flux trace follows immediately from (3.31) and (3.29):

$$
\widehat{\boldsymbol{q}}_{h} \cdot \boldsymbol{n}=-\ell_{h} .
$$

It also follows from Proposition 3.6 that the IP method of (3.30) is well defined when $\alpha>0$ is sufficiently small, a result already established in [38].

Let us end by pointing out that other IP-H-like methods can be obtained. For example, we could take $\boldsymbol{V}(K)=\mathcal{P}_{k-1}(K)^{n}$.

3.7. The NC-H methods. We now consider nonconforming hybridizable (NC-H) methods and show that methods like the $\mathrm{P}_{1}$-nonconforming method introduced in [36] in the framework of the stationary Stokes equations, are in fact $\mathrm{NC}-\mathrm{H}$ methods.

Again the main components of the NC-H method are defined as:

1. For any $k \geq 1$, set

$$
\begin{aligned}
\boldsymbol{V}(K) & =\mathcal{P}_{k-1}(K)^{n}, \quad W(K)=\mathcal{P}_{k}(K), \\
M(\partial K) & =\left\{q:\left.q\right|_{e} \in \mathcal{P}_{k-1}(e) \text { for every face } e \text { of } K\right\}, \\
\boldsymbol{T}(\partial K) & =\left\{q \boldsymbol{n}_{K}:\left.q\right|_{e} \in \mathcal{P}_{k-1}(e) \text { for every face } e \text { of } K\right\} ;
\end{aligned}
$$

2. Define the local solutions $\left(\mathbf{Q m}, \mathfrak{U} \mathrm{m},(\widehat{\mathbf{Q}} \mathrm{m})_{K}\right)$ and $\left(\mathbf{Q} f, \mathcal{U} f,(\widehat{\mathbf{Q}} f)_{K}\right)$ as the elements of $\boldsymbol{V}(K) \times W(K) \times$ $\boldsymbol{T}(\partial K)$ satisfying

$$
\begin{aligned}
& (c \mathbf{Q m}, \boldsymbol{v})_{K}-(U \mathrm{~m}, \operatorname{div} \boldsymbol{v})_{K}=-\langle\mathrm{m}, \boldsymbol{v} \cdot \boldsymbol{n}\rangle_{\partial K}, \forall \boldsymbol{v} \in \boldsymbol{V}(K) \\
- & (\operatorname{grad} w, \mathbf{Q m})_{K}+\langle w, \widehat{\mathbf{Q}} \mathrm{m} \cdot \boldsymbol{n}\rangle_{\partial K}+(d U \mathrm{~m}, w)_{K}=0, \forall w \in W(K) \\
& \langle U \mathrm{~m}, \mu\rangle_{\partial K}=\langle\mathrm{m}, \mu\rangle_{\partial K}, \forall \mu \in M(\partial K)
\end{aligned}
$$

and

$$
\begin{aligned}
& (c \mathbf{Q} f, \boldsymbol{v})_{K}-(\mathcal{U} f, \operatorname{div} \boldsymbol{v})_{K}=0, \forall \boldsymbol{v} \in \boldsymbol{V}(K) \\
- & (\operatorname{grad} w, \mathbf{Q} f)_{K}+\langle w, \widehat{\mathbf{Q}} f \cdot \boldsymbol{n}\rangle_{\partial K}+(d \mathcal{U} f, w)_{K}=(f, w), \forall w \in W(K) \\
& \langle\mathcal{U} f, \mu\rangle_{\partial K}=0, \forall \mu \in M(\partial K) .
\end{aligned}
$$

3. The space of approximate traces is given by $\mathrm{M}_{h}=\mathcal{M}_{h, k-1}$.

Having completed the definition of the main ingredients of the method, we now verify the assumptions of Theorem 2.4 .

Sufficient conditions under which Assumption 2.1 on the existence and uniqueness of the local solvers holds are given next.

Proposition 3.9. For $k=1$ and arbitrary $n$, and for odd $k>1$ and $n=2$, the local solvers (3.33) and (3.34) have unique solutions.

Proof. We only prove the result for the first local solver since the other can be proven in a similar way. Since (3.33) is a square system, it suffices to prove that if $\mathrm{m}=0$ then $\mathbf{Q m}=0, \mathcal{U} \mathrm{m}=0$, and $\widehat{\mathbf{Q}} \mathrm{m}=0$. Choosing $\boldsymbol{v}=\mathbf{Q m}$ and $w=\mathcal{U} \mathrm{m}$, adding (3.33a) and (3.33b), and integrating by parts, we get

$$
(c \mathbf{Q m}, \mathbf{Q m})_{K}+(d \mathcal{U} \mathrm{m}, \mathcal{U} \mathrm{m})_{K}+\langle\mathcal{U} \mathrm{m},(\widehat{\mathbf{Q}} \mathrm{m}-\mathbf{Q m}) \cdot \boldsymbol{n}\rangle_{\partial K}=0 .
$$

If $\mathrm{m}=0$, the equation $(3.33 \mathrm{c})$ implies that $\langle\mathcal{U} \mathrm{m}, \mu\rangle_{\partial K}=0$ for all $\mu \in M(\partial K)$. Since $(\widehat{\mathbf{Q}} \mathrm{m}-\mathbf{Q} \mathrm{m}) \cdot \boldsymbol{n} \in M(\partial K)$ then the last term on the left hand side above is zero and hence $\mathbf{Q m}=0$ and $d \mathcal{U} \mathrm{m}=0$. Substituting this into (3.33a), we have

$$
0=(\mathcal{U m}, \operatorname{div} \boldsymbol{v})_{K}=-(\operatorname{grad} U \mathrm{~m}, \boldsymbol{v})_{K} \quad \text { for all } \boldsymbol{v} \in \mathcal{P}_{k-1}(K)^{n}
$$


where while integrating by parts we have again used that $\langle\mathcal{U m}, \boldsymbol{v} \cdot \boldsymbol{n}\rangle_{\partial K}=0$. Thus $\operatorname{grad} \mathcal{U}$ vanishes, so $\mathcal{U m}_{\mathrm{m}}$ is a constant function, and $\langle\mathcal{U m}, \mu\rangle_{\partial K}=0$ implies that it vanishes identically.

It remains to show that $\widehat{\mathbf{Q}} \mathrm{m} \cdot \boldsymbol{n}$ also vanishes. Since both $\mathbf{Q m}$ and $d U_{\mathrm{m}}$ vanish, (3.33b) implies that

$$
\langle w, \widehat{\mathbf{Q}} \mathrm{m} \cdot \boldsymbol{n}\rangle_{\partial K}=0 \quad \text { for all } w \in \mathcal{P}_{k}(K) .
$$

For $k=1$, that is, for Crouzeix-Raviart nonconforming finite elements, the result follows easily for any dimension $n \geq 2$. Indeed, let $\left.\widehat{\mathbf{Q}} \mathrm{m} \cdot \boldsymbol{n}\right|_{e_{j}}=a_{j}$, for some constants $a_{j}, j=1, \ldots, n+1$. Let $w \in \mathcal{P}_{1}(K)$ be a linear function on $K$ which takes values $a_{j}$ at the centroids of the faces $e_{j}$ of $K, j=1, \ldots, n+1$. Then $0=\langle w, \widehat{\mathbf{Q}} \mathrm{m} \cdot \boldsymbol{n}\rangle_{\partial K}=$ $\sum_{j=1}^{n+1}\left|e_{j}\right| a_{j}^{2}$ implies $a_{j}=0$ for all faces, that is, $\widehat{\mathbf{Q}} \mathbf{m} \cdot \boldsymbol{n}=0$.

Finally, we show the same for $k$ odd and $n=2$. Let $e_{1}, e_{2}$, and $e_{3}$ denote the three edges of $K$ and let $L_{i}^{(j)}$ denote the $i^{\text {th }}$ Legendre polynomial mapped affinely to $e_{j}$ from $[-1,1]$. Assume that the first vertex of the edge $e_{j}$ is mapped to the point -1 and that, as we go from its first to its second vertex, the triangle $K$ is to our left. Since $\left.\widehat{\mathbf{Q}} \mathbf{m} \cdot \boldsymbol{n}\right|_{e_{j}} \in \mathcal{P}_{k-1}\left(e_{j}\right)$, we can write

$$
\left.(\widehat{\mathbf{Q}} \mathrm{m})_{K} \cdot \boldsymbol{n}_{K}\right|_{e_{j}}=\sum_{i=0}^{k-1} a_{i}^{(j)} L_{i}^{(j)}
$$

Note that when $i$ is even, $L_{i}^{(j)}$ takes the same value at the endpoints of $e_{j}$. Therefore, for any even $i$, we can choose a $w$ in (3.35) such that $\left.w\right|_{e_{1}}=L_{i}^{(1)},\left.w\right|_{e_{2}}=-L_{k}^{(2)}$, and $\left.w\right|_{e_{3}}=L_{k}^{(3)}$ (because with these choices $\left.w\right|_{\partial K}$ is continuous). Then (3.35) implies that the coefficient $a_{i}^{(1)}$ vanishes. Repeating the argument for all edges, we find that $a_{i}^{(j)}=0$ for all even $i$ and $j=1,2,3$. Next, for odd $i$, choose $w$ such that $\left.w\right|_{e_{1}}=L_{i}^{(1)},\left.w\right|_{e_{2}}=L_{i-1}^{(2)}$, and $\left.w\right|_{e_{3}}=-L_{k}^{(3)}$. Since $k$ is odd, these choices make $\left.w\right|_{\partial K}$ continuous, so such a $w$ can be found. With this $w,(3.35)$ now gives that $a_{i}^{(1)}=0$ for all odd $i$ as well. Repeating this argument for other edges, we find all coefficients to be zero, so $\widehat{\mathbf{Q}} \mathrm{m}$ vanishes.

The conservativity condition (2.6) with $\mathrm{M}_{h}=\mathcal{M}_{h, k-1}$ clearly implies strong conservativity. Using Theorem 2.1 and noting that the unknown fluxes $\widehat{\mathbf{Q}}$. cancel off in the weak formulation (2.9), by the boundary condition (3.33c) for the local solver, we have that the bilinear form is symmetric:

$$
a_{h}(\eta, \mu)=(c \mathbf{Q} \eta, \mathbf{Q} \mu)_{\mathcal{T}_{h}}+(d \mathcal{U} \eta, \mathcal{U} \mu)_{\mathcal{T}_{h}} .
$$

Its positive definiteness will follow from Theorem 2.4 once Assumptions 2.2 and 2.3 are verified, which we do next.

Proposition 3.10. Assumption 2.2 on the positive semidefiniteness of the local solvers and Assumption 2.3, the gluing condition, are satisfied with $M(\partial K)$ defined as in (3.32).

Proof. First, we show that the condition (2.13a) holds. Taking $\boldsymbol{v}=\mathbf{Q m}$ in (3.33a), $w=\boldsymbol{U}_{\mathrm{m}}$ in (3.33b), and adding the equations, we get, after a few simple algebraic manipulations, that

$$
\begin{aligned}
-\langle\mathrm{m}, \widehat{\mathbf{Q}} \mathrm{m} \cdot \boldsymbol{n}\rangle_{\partial K} & =(c \mathbf{Q m}, \mathbf{Q m})_{K}+(d \mathcal{U} \mathrm{m}, \mathcal{U} \mathrm{m})_{K}+\langle(\widehat{\mathbf{Q}} \mathrm{m}-\mathbf{Q m}) \cdot \boldsymbol{n}, \mathcal{U} \mathrm{m}-\mathrm{m}\rangle_{\partial K} \\
& =(c \mathbf{Q m}, \mathbf{Q m})_{K}+(d \mathcal{U}, \mathfrak{U})_{K},
\end{aligned}
$$

by the boundary condition (3.33c) for the local solver. This implies that (2.13a) of Assumption 2.2 is satisfied.

Now, we prove the condition (2.13b). If $\langle\mathrm{m}, \widehat{\mathbf{Q}} \mathrm{m} \cdot \boldsymbol{n}\rangle_{\partial K}=0$, then $\left.\mathbf{Q m}\right|_{K}=0$ and the equation (3.33a) becomes

$$
(\operatorname{grad} U \mathrm{~m}, \boldsymbol{v})_{K}=\left\langle U_{\mathrm{m}}-\mathrm{m}, \boldsymbol{v} \cdot \boldsymbol{n}\right\rangle_{\partial K}=0 \quad \text { for all } \boldsymbol{v} \in \boldsymbol{V}(K),
$$

This implies that that $\mathcal{U} \mathrm{m}$ is a constant. This shows that condition (2.13b) of Assumption 2.2 is satisfied.

Assumption 2.3 is trivially satisfied and this completes the proof.

In the Tables 3.1 and 3.2, we give the simplified weak formulation of the $\mathrm{NC}-\mathrm{H}$ method under the further assumption that $c(\boldsymbol{x})$ is a constant matrix on each $K$ in $\mathcal{T}_{h}$. In this case, we can show that the original NC method is an $\mathrm{NC}-\mathrm{H}$ method. To see why, first observe that by summing up the last equation of the local solvers, we find that $u_{h}=\mathfrak{U} \lambda_{h}+\mathfrak{U} g_{h}+\mathfrak{U} f$ satisfies

$$
\left\langle\llbracket u_{h} \rrbracket, \mu\right\rangle_{e}=0, \quad \text { for all } \mu \in \mathcal{P}_{k-1}(e),
$$


for all interior faces $e$, so the weak continuity constraints of the discontinuous method are satisfied. Now, (2.12a) and $(2.12 \mathrm{c})$ become $\left(c \mathbf{Q} \lambda_{h}+\operatorname{grad} \mathcal{U} \lambda_{h}, \boldsymbol{v}\right)_{\mathcal{T}_{h}}=0$ and $(c \mathbf{Q} f+\operatorname{grad} \mathcal{U} f, \boldsymbol{v})_{\mathcal{T}_{h}}=0$ which gives

$$
\boldsymbol{q}_{h}=\mathbf{Q} \lambda_{h}+\mathbf{Q} g_{h}+\mathcal{Q} f=-a \operatorname{grad}\left(\mathcal{U} \lambda_{h}+\mathcal{U} g+\mathcal{U} f\right)=-a \operatorname{grad} u_{h} .
$$

Then (2.8a) implies

$$
\left(a \operatorname{grad} u_{h}, \operatorname{grad} v_{h}\right)_{\mathcal{T}_{h}}+\left(d u_{h}, v_{h}\right)_{\mathcal{T}_{h}}=\left(f, v_{h}\right),
$$

for all $v_{h} \in\left\{w: w \in W_{h},\langle\llbracket w \rrbracket, \mu\rangle_{\varepsilon_{h}^{\circ}}=0\right.$ for all $\mu \in M_{h}$ and $\langle w, \mathrm{~m}\rangle_{\partial \Omega}=0$ for all $\left.\mathrm{m} \in \mathrm{M}_{\mathbf{h}}\right\}$, which is the familiar primal form of this non-conforming method. Note that although the information in $g_{h}$ disappears from the right hand side above, it is contained in $u_{h}$ as $u_{h}=\mathfrak{U} \lambda_{h}+\mathcal{U} g_{h}+\mathcal{U} f$.

Let us end this subsection by pointing out that in the case of lowest order polynomials, $k=1$, and for the case in which $d=0$, and both $c$ and $f$ are constant on each simplex $K$ of the triangulation $\mathcal{T}_{h}$, our hybridization framework allows us to recover a well-known relationship between the RT method of lowest degree and the nonconforming method, [4, 47]. Let us sketch how to obtain it. In this case, we can easily show that the local solver $\mathbf{Q m}$ is the same for both this non-conforming method and that of the RT method of lowest degree; see the computation of the RT method in [26]. Since we also have that $\widehat{\mathbf{Q}} \mathbf{m} \cdot \boldsymbol{n}=\mathbf{Q m} \cdot \boldsymbol{n}$, we can conclude that the stiffness matrix associated with the bilinear form $a_{h}(\cdot, \cdot)$ of both methods is also the same- if the degrees of freedom for the numerical traces are the barycenters of the faces. Moreover, since the average on each simplex of the local solver $\mathcal{U}$ coincides with the local solver $\mathcal{U}$ of the RT method under consideration, the matrix associated with the linear form $b_{h}(\cdot)$ is also the same for both method. Of course, in both cases, we take $g_{h}$ at the barycenter of each face $e \in \mathcal{E}_{h}^{\partial}$ to be the average of $g$ on the face $e$. By Theorem 2.1, the degrees of freedom of the approximate traces are the same for both methods. The above-mentioned relation between the two methods now easily follows from the definition of the approximate solutions (2.5).

4. Other novel methods. In this section, we build on the work done in the previous section and construct what are perhaps the three most important examples methods of the unifying framework. The first is a class of methods employing different local solvers in different parts of the domain which can easily deal with nonconforming meshes. The second is an RT method that can handle hanging nodes. The third is the family of EDG methods; they are constructed from already known hybridized methods in this unified framework in order to reduce their computational complexity. As for the examples of the previous section, we assume that the mesh is simplicial; however, we do not assume it to be necessarily conforming.

4.1. A class of hybridizable methods well suited for adaptivity. We introduce here a class of hybridizable methods able to use different local solvers in different elements and to easily handle nonconforming meshes. They are thus ideal to use with adaptive strategies. After introducing the methods, we prove that they are all well defined. We then discuss their main advantages and give several examples.

To define the methods, we need to specify the numerical fluxes, the local finite element spaces and the space of approximate traces:

1. For any simplex $K \in \mathcal{T}_{h}$ we take

$$
\widehat{\mathbf{Q}} \mathrm{m}=\mathbf{Q} \mathrm{m}+\tau_{K}(U \mathrm{~m}-\mathrm{m}) \boldsymbol{n}, \quad \widehat{\mathbf{Q}} f=\mathbf{Q} f+\tau_{K}(U f) \boldsymbol{n} \quad \text { on } \partial K,
$$

the function $\tau_{K}$ is allowed to change on $\partial K$.

2. The local space $\boldsymbol{V}(K) \times W(K)$ can be any of the following:

$$
\begin{array}{ll}
\left(\mathcal{P}_{k(K)}(K)^{n}+\boldsymbol{x} \mathcal{P}_{k(K)}(K)\right) \times \mathcal{P}_{k(K)}(K) & k(K) \geq 0 \text { and } \tau_{K} \geq 0 \text { on } \partial K, \\
\mathcal{P}_{k(K)}(K)^{n} \times \mathcal{P}_{k(K)-1}(K) & k(K) \geq 1 \text { and } \tau_{K} \geq 0 \text { on } \partial K, \\
\mathcal{P}_{k(K)}(K)^{n} \times \mathcal{P}_{k(K)}(K) & k(K) \geq 0 \text { and } \tau_{K}>0 \text { on at least } \\
& \text { one face } e \text { of } K, \\
\mathcal{P}_{k(K)-1}(K)^{n} \times \mathcal{P}_{k(K)}(K) & k(K) \geq 1 \text { and } \tau_{K}>0 \text { on } \partial K ;
\end{array}
$$

3. The space of approximate traces is

$$
\mathrm{M}_{h}=\mathfrak{M}_{h} \cap\left\{\mu \in L^{2}\left(\mathcal{E}_{h}\right):\left.\mu\right|_{\partial K} \in \mathcal{C}\left(\overline{\left\{\boldsymbol{x} \in \partial K: \tau_{K}(\boldsymbol{x})=\infty\right\}}\right) \quad \forall K \in \mathcal{T}_{h}\right\}
$$


where

$$
\mathfrak{M}_{h}:=\left\{\mu \in L^{2}\left(\mathcal{E}_{h}\right):\left.\mu\right|_{e} \in \mathcal{P}_{k(e)}(e) \text { for all } e \in \mathcal{E}_{h}^{\circ}\right\}
$$

Here, if $e=\partial K^{+} \cap \partial K^{-}$, we set

$$
k(e):= \begin{cases}\max \left\{k\left(K^{+}\right), k\left(K^{-}\right)\right\} & \text {if } \tau^{+}<\infty \text { and } \tau^{-}<\infty, \\ k\left(K^{+}\right) & \text {if } \tau^{+}=\infty \text { and } \tau^{-}<\infty, \\ k\left(K^{-}\right) & \text {if } \tau^{+}<\infty \text { and } \tau^{-}=\infty, \\ \min \left\{k\left(K^{+}\right), k\left(K^{-}\right)\right\} & \text {if } \tau^{+}=\infty \text { and } \tau^{-}=\infty\end{cases}
$$

and take $g_{h}=I_{h} g$ for some interpolation operator $I_{h}$ into $\mathrm{M}_{h}$.

Note that the choice $\tau=\infty$ on some interior faces $e \in \mathcal{E}_{h}^{\circ}$ is allowed. We follow the convention that in this case, the definition of the local solvers has to be modified as for the limiting cases of LDG-H methods, see Subsection 3.4. The definition of the methods is competed with the following assumption on the values of the stabilization parameter $\tau$.

Assumption 4.1. For each element $K \in \mathcal{T}_{h}$ and each interior face $e \in \mathcal{E}_{h}^{\circ}$ on $\left.\partial K \tau_{K}\right|_{e} \in[0, \infty]$ and

$$
\left.\tau_{K}\right|_{e} \in(0, \infty) \text { if } e \text { is not a face of } K \text {. }
$$

Let us briefly discuss this assumption. First, let us recall the difference between an interior face $e \in \mathcal{E}_{h}^{\circ}$ and the faces of the simplexes of the triangulation. Each simplex $K$ in the partition $\mathcal{T}_{h}$ has $n+1$ faces determined by its vertices. On the other hand, if $e$ is an interior face, we have that $e=\partial K^{+} \cap \partial K^{-}$for some elements $K^{+}$and $K^{-}$in $\mathcal{T}_{h}$. We thus see that, for nonconforming meshes, although each interior face $e$ is contained in a face of $K^{+}$and a face of $K^{-}$, it is not necessarily a face of $K^{+}$or $K^{-}$. See an example in Fig. 4.1. The main motivation of the above assumption can now be easily seen. Indeed, take any $K \in \mathcal{T}_{h}$. If $e \subset \partial K$ is a face in $\mathcal{E}_{h}^{\circ}$ which is not a face of $K$, then the above assumption forces us to take the numerical trace corresponding to an LDG-H method; in this way, the nonconformity of the mesh can be dealt with in a very natural way. If, on the contrary, $e$ is actually a face, the assumption allows us to take either $\tau_{K}=0, \tau_{K} \in(0, \infty)$ or even $\tau_{K}=\infty$. In this way, the verification of the Assumptions 2.1, 2.2 and 2.3 becomes extremely easy, as we are going to see next.

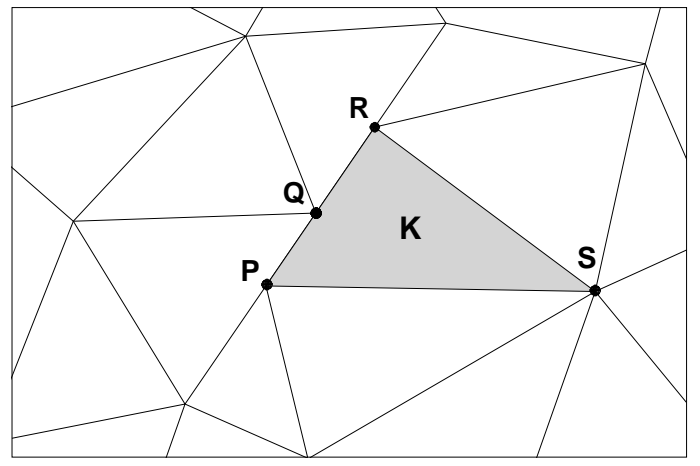

FIG. 4.1. The interior edges $e=\mathbf{P Q}$ and $e=\mathbf{Q R}$ are contained in the face $\mathbf{P R}$ of the element $K$. Assumption 4.1 is satisfied for this element if $\left.\tau_{K}\right|_{\partial K} \in[0, \infty]$, and if $\left.\tau_{K}\right|_{\mathbf{P Q}}$ and $\left.\tau_{K}\right|_{\mathbf{Q R}}$ are taken in $(0, \infty)$.

Next, we show that the approximate solution $\left(\boldsymbol{q}_{h}, u_{h}\right),(2.5)$, provided by this method is well defined.

Proposition 4.1. Consider the method defined by (4.1), (4.2) and (4.3) and let the Assumption 4.1 hold. Then Assumption 2.1 on the existence and uniqueness of the local solvers, Assumption 2.2 on the positive semidefiniteness of the local solvers, and Assumption 2.3, the gluing condition, hold with

$$
\begin{aligned}
M(\partial K)=\{\mu: \quad & \text { on any face } e \in \mathcal{E}_{h}^{\circ} \text { on } \partial K,\left.\quad \mu\right|_{e} \in \mathcal{P}_{k(K)}(e) \text { if }\left.\tau_{K}\right|_{e}=0, \\
& \text { and } \left.\left.\mu\right|_{e} \in L^{2}(e) \text { if }\left.\tau_{K}\right|_{e}>0\right\} .
\end{aligned}
$$


Proof. Thanks to Theorem 2.4, we only have to satisfy Assumptions 2.1, 2.2 and 2.3. We begin by verifying Assumption 2.1 on the existence and uniqueness of the local solvers. Let $K$ be an arbitrary simplex of the triangulation $\mathcal{T}_{h}$. Then, as discussed above, by the condition (4.4), we either have $\tau_{K}=0, \tau_{K} \in(0, \infty)$ or $\tau_{K}=\infty$, on each of the faces of each simplex $K$ of the triangulation $\mathcal{T}_{h}$. As a consequence, the fact that the local solvers are well defined can be easily obtained by a straightforward modification of the proofs of similar results for the LDG-H methods, Proposition 3.5, and the CG-H method, Proposition 3.5. For this reason, we do not present here the proof. However, let us note that whenever $\left.\tau_{K}\right|_{e}=\infty$, we strongly impose a Dirichlet boundary condition and so the space of approximate traces restricted to $\partial K$ and the local space $W(K)$ must satisfy the following compatibility condition:

$$
\left\{\left.\mu\right|_{S}: \quad \mu \in \mathrm{M}_{h}\right\} \subset\left\{\left.w\right|_{S}: \quad w \in W(K)\right\} \quad \text { where } \quad S:=\overline{\left\{\boldsymbol{x} \in \partial K: \tau_{K}(\boldsymbol{x})=\infty\right\}} .
$$

This condition can be easily verified by noting that, if $\tau=\infty$ on the interior face $e \in \mathcal{E}_{h}^{\circ}$, then $e$ must be a face of $K$ by the conditions on the stabilization parameters (4.4), and since, by the definition of $k(e),(4.3 \mathrm{c})$, we have that $k(e) \leq k(K)$.

Next, let us prove that Assumption 2.2 on the positive semidefiniteness of the local solvers is satisfied with $M(\partial K)$ as in (4.5). For the choice (4.2a), it is easy to see that it follows from Proposition 3.1 and from the definition of $k(e),(4.3 \mathrm{c})$. For the remaining choices, the result follows from Proposition 3.3 and the definition of $k(e),(4.3 \mathrm{c})$.

The Assumption 2.3, the gluing condition, also follows by using the arguments of the previous section. Indeed, for an interior face $e=\partial K^{+} \cap \partial K^{-}$, if $\tau^{+}$or $\tau^{-}$is positive, the result trivially follows from the condition (4.3c) and the fact that on $e$, one of the projections $P_{\partial K^{+}}$or $P_{\partial K^{-}}$becomes the identity by the definition of $k(e),(4.3 \mathrm{c})$. It remains to consider the case $\tau^{+}=\tau^{-}=0$. By (4.3c), either $k\left(K^{+}\right)$or $k\left(K^{-}\right)$ equals $k(e)$, say $k(e)=k\left(K^{+}\right)$. Then we immediately have that $P_{\partial K^{+}} \mu=\mu$. This completes the proof. $\square$

Next, let us discuss the main features of these methods:

(i) Variable degree approximation spaces on conforming meshes. The RT-H, BDM-H, and LDG-H methods considered in the previous section used a single local solver in each of the elements $K$ of the conforming triangulation $\mathcal{T}_{h}$. A variable-degree version of each of these methods is a particular case of the class of methods presented here. Note that the case of the variable degree RT method, introduced and analyzed in [27], is exactly the variable-degree version of the method using the RT method as local solvers.

(ii) Automatic coupling of different methods on conforming meshes. The methods presented here allow for the use of different local solvers in different elements $K$ of $\mathcal{T}_{h}$ which are then automatically coupled. For example, if we are working with the RT, LDG and CG local solvers, the conservativity condition implicitly imposes the following expressions for the numerical traces:

$$
\begin{aligned}
& \widehat{u}_{h}=\left.u_{h}\right|_{\Omega_{\mathrm{LDG}}}+\frac{1}{\tau_{\mathrm{LDG}}} \llbracket \boldsymbol{q}_{h} \rrbracket, \quad \widehat{\boldsymbol{q}}_{h}=\left.\boldsymbol{q}_{h}\right|_{\Omega_{\mathrm{RT}}} \quad \text { (coupling RT and LDG), } \\
& \widehat{u}_{h}=\left.u_{h}\right|_{\Omega_{\mathrm{CG}}}, \quad \quad \widehat{\boldsymbol{q}}_{h}=\left.\boldsymbol{q}_{h}\right|_{\Omega_{\mathrm{LDG}}}+\tau_{\mathrm{LDG}} \llbracket u_{h} \rrbracket \quad \text { (coupling of LDG and CG), } \\
& \widehat{u}_{h}=\left.u_{h}\right|_{\Omega_{\mathrm{CG}}}, \quad \quad \widehat{\boldsymbol{q}}_{h}=\left.\boldsymbol{q}_{h}\right|_{\Omega_{\mathrm{RT}}} . \quad \text { (coupling of CG and RT). }
\end{aligned}
$$

Note that this coupling holds even for nonconforming meshes.

It is interesting to compare the above couplings with other couplings in the available literature, namely,

$$
\begin{array}{lll}
\widehat{u}_{h}=\left.u_{h}\right|_{\Omega_{\mathrm{LDG}}}, & \widehat{\boldsymbol{q}}_{h}=\left.\boldsymbol{q}_{h}\right|_{\Omega_{\mathrm{RT}}}+C_{11} \llbracket u_{h} \rrbracket & \text { (coupling of RT and LDG in [23]), } \\
\widehat{u}_{h}=\left.u_{h}\right|_{\Omega_{\mathrm{CG}}}, & \widehat{\boldsymbol{q}}_{h}=\left.\boldsymbol{q}_{h}\right|_{\Omega_{\mathrm{LDG}}}+\tau_{\mathrm{LDG}} \llbracket u_{h} \rrbracket & \text { (coupling of LDG and CG in [48]). }
\end{array}
$$

(iii) Mortaring capabilities (for nonconforming meshes). One of the advantageous features of DG methods is their ability to handle nonconforming meshes; see [52] for an application to structural mechanics. The methods under consideration incorporate this mortaring ability thanks to the very form that the numerical trace of the flux on $\partial K$ takes on an interior face $e \in \mathcal{E}_{h}^{\circ}$ which is not a face of $K$ and thanks to the definition of the stabilization parameter $\tau$ therein. Let us give two examples.

If we have a conforming mesh, we can take the first choice of local spaces (4.2a) and set $\tau \equiv 0$. The resulting method, as we have seen, is nothing but the $\mathrm{RT}-\mathrm{H}$ method. We can easily modify this method to handle nonconforming meshes by simply taking $\tau_{K} \in(0, \infty)$ on every interior face $e \in \mathcal{E}_{h}^{\circ}$ which is not a face of $K$, and otherwise, taking $\tau_{K}=0$. Thus, the resulting method can be considered as a variation of the RT method which is capable of handling nonconforming meshes. 
We can do something similar with the CG method. Indeed, if the mesh is conforming, we can take the last choice of local spaces $(4.2 \mathrm{~d})$ and set $\tau \equiv \infty$ to obtain the hybridized CG method. For nonconforming meshes, we can slightly modify the method by simply taking $\tau_{K} \in(0, \infty)$ on every interior face $e \in \mathcal{E}_{h}^{\circ}$ which is not a face of $K$, and otherwise, taking $\tau_{K}=\infty$. The resulting method is thus a variation of the CG method capable of handling nonconforming meshes. It constitutes an alternative to the coupling of the CG and the LDG method proposed in [48] to deal with non-matching meshes.

(iv) The conservativity condition. Let us end by noting that the stiffness matrix associated to the approximate trace $\lambda_{h}$ is always symmetric and positive definite. Moreover, on the interior faces on which $\tau<\infty$, the conservativity condition is enforced strongly.

4.2. The RT method on meshes with hanging nodes. Consider the case of the variable degree RT-H method. The method is obtained by taking the numerical traces as in (4.1) with $\tau \equiv 0$, the local space (4.2a) and the multiplier space as in (4.3). This method does not belong to the family of methods described in the previous subsection because our choice of stabilization parameter does not satisfy the condition (4.4). Thus, to ensure the existence and uniqueness of the approximate solution, we have to impose special conditions on the meshes and link the definition of $k(K)$ to the structure of the mesh.

Let us illustrate how to do this in the two-dimensional case. The meshes $\mathcal{T}_{h}$ we consider are constructed as follows. First, construct a conforming triangulation of $\Omega, \mathcal{T}_{h}^{(0)}:=\left\{K^{(0)}\right\}$. Then take a subset of that triangulation, $\mathcal{T}_{h}{ }^{(0,1)}$, and divide each of its triangles into four congruent triangles; the set of those triangles is denoted by $\mathcal{T}_{h}{ }^{(1)}$. Next, for $j=2, \ldots, \ell$, given the set $\mathcal{T}_{h}{ }^{(j-1)}$, pick the subset $\mathcal{T}_{h}{ }^{(j-1, j)}$ and create the set of smaller triangles $\mathcal{T}_{h}{ }^{(j)}$. The simple case $\ell=1$ is illustrated on Fig. 4.2. Finally, we establish a link between the mesh and the definition of the polynomial degree of the RT method on the triangle $K, k(K)$, as follows. If $e=\partial K^{+} \cap \partial K^{-}$, we take $k(e):=\max \left\{k\left(K^{+}\right), k\left(K^{-}\right)\right\}$and require that

$$
\text { if } e \text { is not an edge of } K^{-} \text {, then } k\left(K^{+}\right) \geq k\left(K^{-}\right) \text {. }
$$

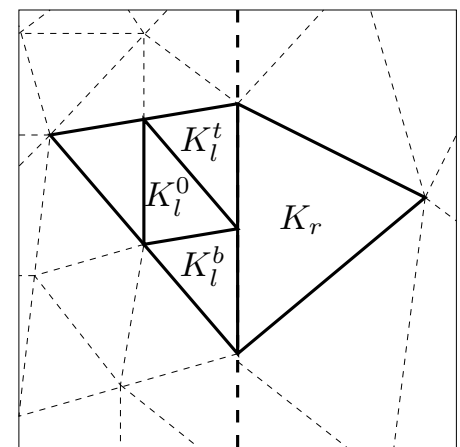

FIG. 4.2. In the presence of hanging nodes like the above, an RT-H method with the spaces on edges chosen to have the maximum degree from either side is well defined if $k\left(K_{l}^{t}\right) \geq k\left(K_{r}\right)$ and $k\left(K_{l}^{b}\right) \geq k\left(K_{r}\right)$. The degree $k\left(K_{l}^{0}\right)$ can be arbitrarily chosen.

Next, we show that the method is well defined.

Proposition 4.2. The variable-degree RT-H method on meshes with hanging nodes as described above is uniquely solvable.

Proof. If we proceed exactly as in Proposition 3.1, we can see that Assumption 2.1 on the existence and uniqueness of the local solvers is verified and that Assumption 2.2 on the positive semidefiniteness of the local solvers is also verified provided we change the definition of the set $M(\partial K)$ to

$$
M(\partial K)=\left\{\mu:\left.\mu\right|_{e} \in \mathcal{P}_{k(e)}(e) \text { for all edges } e \text { of } \partial K\right\} .
$$

The result follows if we prove that there is only one solution $\lambda_{h} \in M_{h}$ of the weak formulation (1.7).

To do that, we proceed exactly as in the proof of Theorem 2.4. First, since Assumption 2.1 holds, we have that the system (2.9) is well defined. Next we show that $a_{h}(\mu, \mu)=0$ for $\mu \in M_{h}$ implies $\mu=0$. By Assumption 2.2 , we readily obtain that, for any given $K \in \mathcal{T}_{h}$, we have that, on $\partial K$,

$$
C_{K}=P_{\partial K} \mu,
$$


where $P_{\partial K}$ is the $\mathrm{L}^{2}$-projection into $M(\partial K)$ as defined above. It remains to show that this implies that $\mu$ is a constant on $\mathcal{E}_{h}$. To do that, we use the structure of the meshes and the definition of $k(K)$ for all $K \in \mathcal{T}_{h}$.

We proceed as follows. We claim that, for $j=\ell, \ell-1, \ldots, 0$, we have that $\left.\mu\right|_{\partial K}$ is a constant for all $K \in \mathcal{T}_{h}{ }^{(j)}$. This immediately implies that $\mu$ is a constant on $\mathcal{E}_{h}$ and, since $\left.\mu\right|_{\partial \Omega}=0$, that $\mu=0$ on $\mathcal{E}_{h}$.

It remains to prove the claim. We proceed by induction on $j$. Let us prove the inductive hypothesis for $j=\ell$. Let $K$ be any triangle in $\mathcal{T}_{h}{ }^{(\ell)}$ and pick any of its edges, $e$. If the edge $e$ lies on $\partial \Omega$, we immediately have that $\mu=C_{K}=0$. If $e=\partial K \cap \partial K^{\prime}$ for some triangle $K^{\prime} \in \mathcal{T}_{h}{ }^{(\ell)}$, we proceed as in the proof of Theorem 2.4 to conclude that

$$
\mu=C_{K}=C_{K^{\prime}} \quad \text { on } e .
$$

The only other remaining possibility, by construction of the triangulation $\mathcal{T}_{h}{ }^{(\ell)}$, is that $e=\partial K \cap \partial K^{\prime}$ for some triangle $K^{\prime} \in \mathcal{T}_{h}^{\left(\ell^{\prime}\right)}$, with $\ell^{\prime}<\ell$. In this case, $e$ is not a edge of $K^{\prime}$ and, by the condition (4.6) on $k(K)$, we have that $k(K) \geq k\left(K^{\prime}\right)$ and hence $k(e):=\max \left\{k(K), k\left(K^{\prime}\right)\right\}=k(K)$. This implies that

$$
\mu=C_{K} \quad \text { on } e .
$$

Since the edge $e$ was picked arbitrarily, we conclude that $\left.\mu\right|_{\partial K}$ is a constant, as wanted.

Now, let us assume that the inductive hypothesis holds for $j=J$ and let us prove it also holds for $j=J-1$. Let $K$ be any triangle in $\mathcal{T}_{h}^{(J-1)}$ and pick any of its edges, $e$. Since by the inductive hypothesis, $\left.\mu\right|_{\partial K}$ is a constant for all $K \in \mathcal{T}_{h}^{(J)}$, we have that $\left.\mu\right|_{\partial K}$ is a constant for all $K \in \mathcal{T}_{h}^{(J-1, J)}$ since, by construction, each of the triangles in $\mathcal{T}_{h}{ }^{(J-1, J)}$ is subdivided in four congruent triangles in $K \in \mathcal{T}_{h}{ }^{(J)}$. Hence $\mu=C_{K}$ on $e$ if the edge $e$ lies in the border of any triangle in $K \in \mathcal{T}_{h}{ }^{(J-1, J)}$. To finish the proof, we only need to prove the same result in the remaining three cases: (i) if the edge $e$ lies on $\partial \Omega$, (ii) if $e=\partial K \cap \partial K^{\prime}$ for some triangle $K^{\prime} \in \mathcal{T}_{h}{ }^{(J-1)} \backslash \mathcal{T}_{h}^{(J-1, J)}$, and (iii) if $e=\partial K \cap \partial K^{\prime}$ for some triangle $K^{\prime} \in \mathcal{T}_{h}{ }^{\left(J^{\prime}-1\right)}$, with $J^{\prime}<J$. This can be done exactly as in the previous step. $\square$

4.3. The EDG methods. Now we show that new methods [33] can be immediately generated from already existing hybridized methods by simply reducing the space of their approximate traces. The main interest of these EDG methods, introduced in the setting of shells problems in [43], stems from the further reduction in globally coupled unknowns achieved by reducing the approximate trace space $\mathrm{M}_{h}$.

To construct such methods, we begin with selecting any method defined by uniquely solvable local problems (2.3), (2.4) and a conservativity condition (2.6) yielding a unique approximate trace $\lambda_{h}$. Then by Theorem 2.1, $\lambda_{h} \in M_{h}$ is the only solution of the weak formulation

$$
a_{h}\left(\lambda_{h}, \mu\right)=b_{h}\left(I_{h} g ; \mu\right) \quad \forall \mu \in M_{h},
$$

where we are writing $b_{h}\left(I_{h} g ; \mu\right)$ instead of just $b_{h}(\mu)$ in order to stress its dependency on $I_{h} g \in \mathrm{M}_{h}$. We now define an EDG method by replacing the original approximate trace space $\mathrm{M}_{h}$ by a subspace $\widetilde{\mathrm{M}}_{h}$. This forces us to replace $I_{h} g \in \mathrm{M}_{h}$ by $\widetilde{I}_{h} g \in \widetilde{\mathrm{M}}_{h}$ and to change the conservativity condition, but the local solvers remain the same. Now, define the operator $\mathcal{J}_{h}: \widetilde{M}_{h} \rightarrow \mathrm{M}_{h}$ as the identity operator representing the natural embedding of $\widetilde{\mathrm{M}}_{h}$ into $\mathrm{M}_{h}$, hence the name of these methods, and set

$$
\widetilde{M}_{h}:=\left\{\widetilde{\mu} \in \widetilde{M}_{h}: \widetilde{\mu}=0 \text { on } \partial \Omega\right\} .
$$

Then by Theorem 2.1, the new conservativity condition is equivalent to

$$
a_{h}\left(\mathcal{J}_{h} \widetilde{\lambda}_{h}, \mathcal{J}_{h} \widetilde{\mu}\right)=b_{h}\left(\mathcal{J}_{h} \widetilde{I}_{h} g ; \mathcal{J}_{h} \widetilde{\mu}\right) \quad \forall \widetilde{\mu} \in \widetilde{M}_{h},
$$

where $\widetilde{\lambda}_{h} \in \widetilde{M}_{h}$ is the new approximate trace. Note that we have that $\left.\mathcal{J}_{h} \widetilde{\mu}\right|_{\partial \Omega}=0$ for all $\widetilde{\mu} \in \widetilde{M}_{h}$.

To show that this EDG method is well defined, it suffices to prove that the homogeneous equation (4.8) has only a trivial solution. For simplicity, let us assume that $a_{h}(\cdot, \cdot)$ is symmetric and positive definite. Thus, taking $\underset{\tilde{\mu}}{\widetilde{\lambda}}=\widetilde{\lambda}_{h}$, we get $a_{h}\left(\mathcal{J}_{h} \widetilde{\lambda}_{h}, \mathcal{J}_{h} \widetilde{\lambda}_{h}\right)=0$. By the positive definiteness of $a_{h}(\cdot, \cdot)$, we have $\mathcal{J}_{h} \widetilde{\lambda}_{h}=0$, which implies $\tilde{\lambda}_{h}=0$. Hence (4.8) is uniquely solvable.

Now, let us show that it is very easy to obtain the equations for the EDG method once those of the original method have been obtained. Denote by $\left[\lambda_{h}\right]$ the vector of the degrees of freedom of the function $\lambda_{h}$ with respect 
to some basis in $M_{h}$. Similarly, denote by $\left[\widetilde{\lambda}_{h}\right]$ the vector of degrees of freedom vector of the function $\widetilde{\lambda}_{h}$ in $\widetilde{M}_{h}$. The equation (4.7) can be written in a matrix form as $A\left[\lambda_{h}\right]=b\left(I_{h} g\right)$, and if $\left[\mathcal{J}_{h} \widetilde{\lambda}_{h}\right]=T\left[\widetilde{\lambda}_{h}\right]$, then the equation for $\left[\widetilde{\lambda}_{h}\right]$ is $T^{t} A T\left[\widetilde{\lambda}_{h}\right]=T^{t} b\left(\mathcal{J}_{h} \widetilde{I}_{h} g\right)$. Here $T$ is the rectangular matrix representing the basis of $\widetilde{M}_{h}$ with respect to basis of $M_{h}$. Since $\widetilde{M}_{h} \subset M_{h}$, if we use the Lagrange basis functions, $T$ is nothing but a connectivity matrix whose entries are zeroes and ones so it is extremely easy to compute.

Note that the above considerations continue to hold if $\partial_{h}$ is any injective operator from $\widetilde{\mathrm{M}}_{h}$ into $\mathrm{M}_{h}$ such that $\left.\mathcal{\partial}_{h} \widetilde{\mu}\right|_{\partial \Omega}=0$ for all $\widetilde{\mu} \in \widetilde{M}_{h}$. Thus new methods can also be created by using spaces $\widetilde{\mathrm{M}}_{h}$ that are not necessarily subspaces of $\mathrm{M}_{h}$. The main task here would be to find the matrix $T$ which represents the basis of $\widetilde{\mathrm{M}}_{h}$ with respect to basis of $\mathrm{M}_{h}$.

Let us give some examples of EDG methods. The first example of an EDG method was proposed in [43]: It is obtained from an LDG-H method using approximations of degree $k$ in each variable by forcing the continuity of the traces. Thus, whereas the functions in the space of approximate traces for the $\mathrm{LDG}-\mathrm{H}$ method $\mathrm{M}_{h}$ are discontinuous on the borders of the elements, the functions of $\widetilde{\mathrm{M}}_{h}$ are continuous therein. This allows the method to be immediately incorporated into commercial codes. On the other hand, this also results in the degradation of the conservativity properties of the EDG method, which only hold weakly. In some cases, this induces a degradation in the approximating properties of the method as recently proven in [32].

Indeed, in that paper it was shown that, when the stabilization parameter $\tau$ is taken to be of order one, the EDG method converges with order $k$ for $\boldsymbol{q}$ and order $k+1$ for $u$. This has to be contrasted with the fact that the original LDG-H method converges with order $k+1$ in both variables; see [33]. Moreover, in this case, the LDG-H has superconvergence properties that allow to compute, in an element-by-element fashion, a new approximation to $u$ converging with order $k+2$; see also [33]. Such property does not hold for the corresponding EDG method. Even more, numerical experiments show that the computational advantage of the EDG method does not compensate for its loss of accuracy. On the other hand, if the stabilization parameter $\tau$ is taken to be of order $h^{-1}$, both the EDG and the LDG-H methods converge with the same orders, namely, $k$ in $\boldsymbol{q}$ and order $k+1$ in $u$.

The second example is associated with the constructions of subspaces $\widetilde{M}_{h}$ of $M_{h}$ that could be required to be very smooth. For example, we could ask that they be not only continuous on $\mathcal{E}_{h}^{\circ}$ but $\mathcal{C}^{1}$-continuous. This might be reasonable to do if the solution is very smooth and varies slowly in $\Omega$.

The third and last example is associated to methods for non-matching grids. Suppose that $\Omega$ is divided into two domains $\Omega_{1}$ and $\Omega_{2}$ independently meshed, and that we are using the variation of the CG method to handle nonconforming methods described in the first subsection. Then, all the interior faces $e$ lying on the interface $\Gamma:=\partial \Omega_{1} \cap \partial \Omega_{2}$ must be computed and used to define the space of traces $\mathrm{M}_{h}$. (This can be done, although it is a computational geometry tour de force, especially in three dimensions; see [52].) To reduce the size of the interface space on $\Gamma$, we can alternately find a subspace of $\mathrm{M}_{h}$ of functions which are polynomials on the interior faces determined by, say, the vertices of the triangulation of $\Omega_{1}$ lying on $\Gamma$.

5. Extensions and generalizations. Although in all examples we have given, only simplicial elements have been considered, this is not essential. Obviously, quadrilateral, prismatic and other elements could be handled easily by DG methods. Furthermore our framework is applicable for mixed methods using other types of elements, see [12].

Note also that the considered DG, mixed, CG and nonconforming finite element methods used to define the local solvers, are not the only choices. Stabilized, Petrov-Galerkin methods, boundary element and even (if possible) the exact solution can be used as local solvers. For example, the hybridization of the discontinuous Petrov-Galerkin method can be found in [18, 19].

In what follows, we sketch how to extend our results to include Neumann boundary conditions and interface transmission conditions. We also extend them to DG methods using other stabilization mechanisms.

5.1. Other boundary and transmission conditions. The hybridization method proposed here can be easily extended to other type of boundary and transmission conditions.

Neumann boundary condition. For example, the case when on part $\partial \Omega_{N}$ of the boundary $\partial \Omega$ the Neumann boundary condition $\boldsymbol{q} \cdot \boldsymbol{n}=q_{N}$ is specified can be incorporated easily in the hybridization procedure. We simply require that the approximate trace $\lambda_{h}$ belongs to

$$
M_{h}=\left\{\mu \in \mathrm{M}_{h}: \quad \mu=0 \text { on } \partial \Omega_{D}\right\},
$$


where $\partial \Omega_{D}:=\partial \Omega \backslash \partial \Omega_{N}$ is the Dirichlet boundary, and replace the conservativity condition (2.6) by

$$
\left\langle\mu, \llbracket \widehat{\mathbf{Q}} \lambda_{h}+\widehat{\mathbf{Q}} g_{h}+\widehat{\mathbf{Q}} f \rrbracket\right\rangle_{\mathcal{E}_{h}}=\left\langle\mu, q_{N}\right\rangle_{\partial \Omega_{N}} \quad \text { for all } \mu \in M_{h} .
$$

Transmission condition. To handle the transmission condition $\llbracket \boldsymbol{q} \rrbracket=t$ on the $(n-1)$-dimensional surface $\Gamma_{t}$, we simply have to write

$$
\left\langle\mu, \llbracket \widehat{\mathbf{Q}} \lambda_{h}+\widehat{\mathbf{Q}} g_{h}+\widehat{\mathbf{Q}} f \rrbracket\right\rangle_{\mathcal{E}_{h}}=\left\langle\mu, q_{N}\right\rangle_{\partial \Omega_{N}}+\langle\mu, t\rangle_{\Gamma_{t}} \quad \text { for all } \mu \in M_{h},
$$

where we are assuming that $\Gamma_{t} \subset \mathcal{E}_{h}$. This case is equivalent to having a right hand side that is a $\delta$-function with a support on $\Gamma_{t}$.

Jump condition. Now, we can add a jump condition $\llbracket u \rrbracket=\boldsymbol{j}$ on the $(n-1)$-dimensional surface $\Gamma_{j}$ where $\boldsymbol{j} \cdot \boldsymbol{n}$ is given. Then we take a triangulation $\mathcal{T}_{h}$ such that $\Gamma_{j} \subset \mathcal{E}_{h}$ and proceed as follows. Since the exact solution is double-valued on $\Gamma_{j}$, that is, since its traces on $\Gamma_{j}$ are $u^{ \pm}:=\left\{\{u\}+1 / 2 \boldsymbol{n}^{ \pm} \cdot \boldsymbol{j}\right.$, we take the approximation to these traces to be $\lambda_{h}+1 / 2 \boldsymbol{n}^{ \pm} \cdot \boldsymbol{j}$ on $\Gamma_{j}$, and define the function $\left(\mathbf{Q m}_{\boldsymbol{j}}, \mathcal{U m}_{\boldsymbol{j}}\right)$ as the solution of the local solver (2.3) with $\mathrm{m}_{\boldsymbol{j}}$ given by

$$
\mathrm{m}_{\boldsymbol{j}}= \begin{cases}\frac{1}{2} \boldsymbol{n}_{K} \cdot \boldsymbol{j} & \text { on } \partial K \cap \Gamma_{j} \\ 0 & \text { elsewhere. }\end{cases}
$$

Then, we simply rewrite the conservativity condition as

$$
\left\langle\mu, \llbracket \widehat{\mathbf{Q}} \lambda_{h}+\widehat{\mathbf{Q}} \mathrm{m}_{\boldsymbol{j}}+\widehat{\mathbf{Q}} g_{h}+\widehat{\mathbf{Q}} f \rrbracket\right\rangle_{\varepsilon_{h}}=\left\langle\mu, q_{N}\right\rangle_{\partial \Omega_{N}}+\langle\mu, t\rangle_{\Gamma_{t}} \text { for all } \mu \in M_{h} .
$$

We see that the global system for $\lambda_{h}$ has the same matrix and a right hand side that incorporates the data related to the boundary and interface conditions. This particular example shows the ease with which the hybridizable methods can handle various types of boundary and transmission conditions for the differential equation.

5.2. Hybridizable DG methods with other stabilization mechanisms. For each finite element $K \in \mathcal{T}_{h}$, the LDG-H method uses on $\partial K$ the numerical trace $\widehat{\boldsymbol{q}}_{h}=\boldsymbol{q}_{h}+\tau\left(u_{h}-\lambda_{h}\right) \boldsymbol{n}$ and the IP-H uses the numerical trace $\widehat{\boldsymbol{q}}_{h}=-a \operatorname{grad} u_{h}+\tau\left(u_{h}-\lambda_{h}\right) \boldsymbol{n}$. However, these are not the only choices for numerical traces we could use to generate stabilization through the difference between $u_{h}$ and $\lambda_{h}$. Indeed, in the unified analysis of DG methods [5], we see that we can also take $\widehat{\boldsymbol{q}}_{h}=\boldsymbol{q}_{h}+\boldsymbol{\alpha}_{r}\left(\left(u_{h}-\lambda_{h}\right) \boldsymbol{n}\right)$, for the BMMPR method [13], and $\widehat{\boldsymbol{q}}_{h}=-a \operatorname{grad} u_{h}+\boldsymbol{\alpha}_{r}\left(\left(u_{h}-\lambda_{h}\right)\right)$, for the BRMPS method [7]. Here, for any $\boldsymbol{\varphi} \in \boldsymbol{L}^{2}(\partial K)$ the vector $\boldsymbol{\alpha}_{r}(\boldsymbol{\varphi})$ is the element of $\boldsymbol{V}(K)$ such that,

$$
\boldsymbol{\alpha}_{r}(\boldsymbol{\varphi})=-\tau \boldsymbol{r}_{e, K}(\boldsymbol{\varphi}) \text { on each face } e \text { of } K, \quad\left(\boldsymbol{r}_{e, K}(\boldsymbol{\varphi}), \boldsymbol{v}\right)_{K}=-\langle\boldsymbol{\varphi}, \boldsymbol{v}\rangle_{e} \text { for all } \boldsymbol{v} \in \boldsymbol{V}(K) .
$$

It is not difficult to verify that results similar to those obtained for the LDG-H and IP-H methods can also be obtained for similar BMMPR-H and BRMPS-H methods, respectively. Let us briefly comment on a couple of interesting details. To fix ideas, we consider the BMMPR-H methods. For these methods, Theorem 2.1 holds with

$$
\begin{aligned}
a_{h}(\eta, \mu) & =(c \mathbf{Q} \eta, \mathbf{Q} \mu)_{\mathcal{T}_{h}}+\left(d \mathcal{U} \eta, \mathcal{U}_{\mu}\right)_{\mathcal{T}_{h}}+\left\langle 1, \llbracket\left(\mathcal{U}_{\mu}-\mu\right)\left(\boldsymbol{\alpha}_{r}\left(\left(\mathcal{U}_{\eta}-\eta\right) \boldsymbol{n}\right)\right) \rrbracket\right\rangle_{\mathcal{E}_{h}} \\
b_{h}(\mu) & =\left\langle g_{h},\left(\mathbf{Q} \mu+\boldsymbol{\alpha}_{r}(\mathcal{U} \boldsymbol{n})\right) \cdot \boldsymbol{n}\right\rangle_{\partial \Omega}+\left(f, \mathcal{U}_{\mu}\right)_{\mathcal{T}_{h}},
\end{aligned}
$$

provided $\left.g_{h}\right|_{\varepsilon_{h}^{\circ}}=0$. It is not difficult to see that the bilinear form $a_{h}(\cdot, \cdot)$ is symmetric. Indeed, we have that

$$
\begin{aligned}
\left\langle(U \mu-\mu) \boldsymbol{n}, \boldsymbol{\alpha}_{r}((U \eta-\eta) \boldsymbol{n})\right\rangle_{\partial K} & =-\left.\sum_{e \text { face of } K} \tau_{K}\right|_{e}\left\langle(\mathcal{U} \mu-\mu) \boldsymbol{n}, \boldsymbol{r}_{e, K}((U \eta-\eta) \boldsymbol{n})\right\rangle_{e} \\
& =+\left.\sum_{e \text { face of } K} \tau_{K}\right|_{e}\left(\boldsymbol{r}_{e, K}((\mathcal{U}-\mu) \boldsymbol{n}), \boldsymbol{r}_{e, K}((U \eta-\eta) \boldsymbol{n})\right)_{K} .
\end{aligned}
$$

The fact that the bilinear form $a_{h}(\cdot, \cdot)$ is positive definite follows from Theorem 2.4 and a slight modification of Proposition 3.3; in it, we take $M(\partial K)=\left\{v:\left.v\right|_{e} \in \mathcal{P}_{k}(e), e \in \partial K\right\}$. Note that Assumption 2.2 is then satisfied since $\boldsymbol{r}_{e, K}(\boldsymbol{\varphi})=\mathbf{0}$ if and only if the $L^{2}$-projection of $\left.\boldsymbol{\varphi}\right|_{e}$ into $\mathcal{P}_{k}(e)$ is zero. 
Finally, note that the conservativity condition is enforced strongly. In this case, however, we do not have an explicit expression of the approximate trace $\lambda_{h}$ in terms of $\left(\boldsymbol{q}_{h}, u_{h}\right)$ as we have for the LDG-H methods in Proposition 3.4. Instead, we only have the relation

$$
\llbracket \boldsymbol{\alpha}_{r}\left(\lambda_{h} \boldsymbol{n}\right) \rrbracket=\llbracket \boldsymbol{\alpha}_{r}\left(u_{h} \boldsymbol{n}\right) \rrbracket+\llbracket \boldsymbol{q}_{h} \rrbracket \quad \text { on } \mathcal{E}_{h}^{\circ}
$$

Let us end by noting that extensions of this work to other problems arising in continuum mechanics, fluid dynamics and electromagnetism constitutes the subject of ongoing work.

Acknowledgments. The first author would like to thank Martin Vohralík for bringing to his attention the reference [21].

\section{REFERENCES}

[1] T. Arbogast and Z. Chen, On the implementation of mixed methods as nonconforming methods for second-order elliptic problems, Math. Comp., 64 (1995), pp. 943-972.

[2] T. Arbogast, L.C. Cowsar, M.F. WheEler, and I. Yotov, Mixed finite element methods on nonmatching multiblock grids, SIAM J. Numer. Anal., 37 ( 4) (1997), pp. 1295-1315

[3] D. N. ARnold, An interior penalty finite element method with discontinuous elements, SIAM J. Numer. Anal., 19 (1982), pp. $742-760$.

[4] D. N. ARnold AND F. BREzZI, Mixed and nonconforming finite element methods: implementation, postprocessing and error estimates, RAIRO Modél. Math. Anal. Numér., 19 (1985), pp. 7-32.

[5] D. N. Arnold, F. Brezzi, B. Cockburn, and L. D. Marini, Unified analysis of discontinuous Galerkin methods for elliptic problems, SIAM J. Numer. Anal., 39 (2002), pp. 1749-1779.

[6] G. A. BAKer, Finite element methods for elliptic equations using nonconforming elements, Math. Comp., 31 (1977), pp. 4559.

[7] F. Bassi, S. Rebay, G. Mariotti, S. Pedinotti, and M. Savini, A high-order accurate discontinuous finite element method for inviscid and viscous turbomachinery flows, in 2nd European Conference on Turbomachinery Fluid Dynamics and Thermodynamics, R. Decuypere and G. Dibelius, eds., Antwerpen, Belgium, March 5-7 1997, Technologisch Instituut, pp. 99-108.

[8] F. Ben Belgacem and Y. Maday, The mortar element method for three dimensional finite elements, $\mathrm{M}^{2} \mathrm{AN}$ Math. Model. Numer. Anal., 31 (1997), pp. 289-302.

[9] C. Bernardi, Y. Maday, and A. T. Patera, Domain Decomposition by the mortar element method, in Asymptotic and Numerical Methods for Partial Differential Equations with Critical Parameters, H.G. Kaper and M. Garbey, eds., Kluwer Academic Publishers (1993), pp. 269-286.

[10] J. H. Bramble and J. Xu, A local post-processing technique for improving the accuracy in mixed finite-element approximations, SIAM J. Numer. Anal., 26 (1989), pp. 1267-1275.

[11] F. Brezzi, J. Douglas, JR., And L. D. Marini, Two families of mixed finite elements for second order elliptic problems, Numer. Math., 47 (1985), pp. 217-235.

[12] F. Brezzi and M. Fortin, Mixed and Hybrid finite element methods, Springer Verlag, 1991.

[13] F. Brezzi, G. Manzini, L. D. Marini, P. Pietra, and A. Russo, Discontinuous finite elements for diffusion problems, in in Atti Convegno in onore di F. Brioschi (Milan 1997), Istituto Lombardo, Accademia di Scienze e Lettere, 1999, pp. 197-217.

[14] A. Buffa, T. J. R. Hughes, And G. Sangalli, Analysis of a multiscale discontinuous Galerkin method for convectiondiffusion problems, SIAM J. Numer. Anal., 44 (2006), pp. 1420-1440 (electronic).

[15] J. Carrero, B. Cockburn, And D. Schötzau, Hybridized, globally divergence-free LDG methods. Part I: The Stokes problem, Math. Comp., 75 (2006), pp. 533-563.

[16] P. Castillo, Performance of discontinuous Galerkin methods for elliptic PDE's, SIAM J. Sci. Comput., 24 (2002), pp. 524547.

[17] P. Castillo, B. Cockburn, I. Perugia, and D. Schötzau, An a priori error analysis of the local discontinuous Galerkin method for elliptic problems, SIAM J. Numer. Anal., 38 (2000), pp. 1676-1706.

[18] P. Causin And R. SACCO, A discontinuous Petrov-Galerkin method with Lagrangian multipliers for second-order elliptic problems, SIAM J. Numer. Anal., 43 (2005), pp. 280-302.

[19] - Static condensation procedures for hybridized mixed finite element methods. Submitted.

[20] F. Celiker And B. Cockburn, Superconvergence of the numerical traces of discontinuous Galerkin and hybridized mixed methods for convection-diffusion problems in one space dimension, Math. Comp., 76 (2007), pp. 67-96.

[21] Z. CHEN, Equivalence between and multigrid algorithms for nonconforming and mixed methods for second-order elliptic problems, East-West J. Numer. Math., 4 (1996), pp. 1-33.

[22] P. Ciarlet, The finite element method for elliptic problems, North-Holland, Armsterdam, 1978.

[23] B. Cockburn and C. Dawson, Approximation of the velocity by coupling discontinuous Galerkin and mixed finite element methods for flow problems, Comput. Geosci. (Special issue: Locally Conservative Numerical Methods for Flow in Porous Media), 6 (2002), pp. 502-522.

[24] B. Cockburn and B. Dong, An analysis of the minimal dissipation local discontinuous Galerkin method for convectiondiffusion problems, J. Sci. Comput. To appear. 
[25] B. Cockburn, B. Dong, and J. Guzmán, A superconvergent LDG-hybridizable Galerkin method for second-order elliptic problems, Math. Comp. To appear.

[26] B. Cockburn and J. Gopalakrishnan, A characterization of hybridized mixed methods for second order elliptic problems, SIAM J. Numer. Anal., 42 (2004), pp. 283-301.

[27] — Error analysis of variable degree mixed methods for elliptic problems via hybridization, Math. Comp., 74 (2005), pp. 1653-1677.

[28] - Incompressible finite elements via hybridization. Part I: The Stokes system in two space dimensions, SIAM J. Numer. Anal., 43 (2005), pp. 1627-1650.

[29] - Incompressible finite elements via hybridization. Part II: The Stokes system in three space dimensions, SIAM J. Numer. Anal., 43 (2005), pp. 1651-1672.

[30] - New hybridization techniques, GAMM-Mitt., 2 (2005), pp. 154-183.

[31] B. Cockburn, J. Gopalakrishnan, And H. Wang, Locally conservative fluxes for the continuous Galerkin method, SIAM J. Numer. Anal., 45 (2007), pp. 1742-1776.

[32] B. Cockburn, J. Guzmán, S.-C. Soon, and H. Stolarski, Analysis of the embedded discontinuous Galerkin method for second-order elliptic problems. Submitted.

[33] B. Cockburn, J. Guzmán, And H. Wang, Superconvergent discontinuous Galerkin methods for second-order elliptic problems, Math. Comp. To appear.

[34] B. Cockburn and C.-W. Shu, The local discontinuous Galerkin method for time-dependent convection-diffusion systems, SIAM J. Numer. Anal., 35 (1998), pp. 2440-2463.

[35] M. Comodi, The Hellan-Herrmann-Johnson method: some new error estimates and postprocessing, Math. Comp., 52 (1989), pp. $17-29$.

[36] M. Crouzeix And P. A. Raviart, Conforming and nonconforming finite element methods for solving the stationary stokes equations, RAIRO Modél. Math. Anal. Numér., 7 (1973), pp. 33-75.

[37] V. Dobrev, R. Lazarov, P. Vassilevski, and L. Zikatanov, Two-level preconditioning of discontinuous Galerkin method for second order elliptic problems, Numer. Linear Alg. Appl., 13 (2006), pp. 753-770.

[38] R. Ewing, J. WAng, AND Y. YANG, A stabilized discontinuous finite element method for elliptic problems, Numer. Linear Alg. Appl., 10 (2003), pp. 83-104.

[39] B. M. FraejIS DE VeUBEKe, Displacement and equilibrium models in the finite element method, in Stress Analysis, O. Zienkiewicz and G. Holister, eds., Wiley, New York, 1977, pp. 145-197.

[40] V. Girault, S. Sun, M. F. Wheeler, And I. Yotov, Coupling Discontinuous Galerkin and Mixed Finite Element Discretizations using Mortar Finite Elements. Submitted.

[41] J. Gopalakrishnan, On the Mortar Finite Element Method, Ph.D. thesis, 1999, Texas A\&M University.

[42] J. Gopalakrishnan, A Schwarz preconditioner for a hybridized mixed method, Comput. Methods Appl. Math., 3 (2003), pp. $116-134$.

[43] S. Güzey, B. Cockburn, and H. Stolarski, The embedded discontinuous Galerkin methods: Application to linear shells problems, Internat. J. Numer. Methods Engrg., 70 (2007), pp. 757-790.

[44] J. T. R. Hughes, G. Scovazzi, P. B. Bochev, And A. Buffa, A multiscale discontinuous Galerkin method with the computational structure of a continuous Galerkin method, Comput. Methods Appl. Mech. Engng., 195 (2006), pp. $2761-2787$.

[45] Y. A. Kuznetsov And M.F. Wheeler, Optimal order substructuring preconditioners for mixed finite element methods on nonmatching grids, East-West J. Numer. Math., (1995), pp. 127-143.

[46] J. L. Lions and E. Magenes, Nonhomogeneous Boundary Value Problems and Applications, Springer-Verlag, Berlin, 1972.

[47] L. D. MARINI, An inexpensive method for the evaluation of the solution of the lowest order Raviart-Thomas mixed method, SIAM J. Numer. Anal., 22 (1985), pp. 493-496.

[48] I. Perugia And D. Schötzau, On the coupling of local discontinuous Galerkin and conforming finite element methods, J. Sci. Comput., 16 (2001), pp. 411-433.

[49] P. A. Raviart and J. M. Thomas, A mixed finite element method for second order elliptic problems, in Mathematical Aspects of Finite Element Method, I. Galligani and E. Magenes, eds., Lecture Notes in Math. 606, Springer-Verlag, New York, 1977, pp. 292-315.

[50] B. Rivière And M. F. Wheeler, Coupling locally conservative methods for single-phase flow, Comput. Geosci. (Special issue: Locally Conservative Numerical Methods for Flow in Porous Media), 6 (2002), pp. 269-284.

[51] J.-E. Roberts And J.-M. Thomas, Mixed and hybrid methods, in Handbook of Numerical Analysis, II, Handb. Numer. Anal., II, North-Holland, Amsterdam, 1991, pp. 523-639.

[52] S. Siddarth, J. Carrero, B. Cockburn, K. Tamma, and R. Kanapady, The local discontinuous Galerkin method and component design integration for 3D elasticity, in Proceedings of the Third M.I.T. Conference on Computational Fluid and Solid Mechanics, Cambridge, MA, USA, K. Bathe, ed., Elsevier, 2005, pp. 492-494.

[53] C. Wieners, B. Wohlmuth, The coupling of mixed and conforming finite element discretizations, Domain Decomposition Methods 10, (J. Mandel, C. Farhat, and X.-C. Cai, eds.), 1997, pp. 453-459, American Mathematical Society vol. 218. 